\title{
i) $\quad 000-3165-6$
}

FUNDAMENTAL STUDIES OF RAPID PLASMA HEATING

BY COLLECTIVE INTERACTIONS, USING STRONG

TURBULENCE AND RELATIVISTIC ELECTRON BEAMS

ANNUAL PROGRESS REPORT

R. Kribe1, C. Ekdahl, P. Korn and C. B. Wharton

\author{
Laboratory of Plasma Studies \\ Cornel1 University \\ Ithaca, New York
}

This report NOT ICE

This report was prepared as an account of work sponsored by the United States Government. Neither the United States nor the United States Atomic Energy Commission, nor any of their employees, nor any of their contractors, subcontractors, or their employees, makes any warranty, express or implied, or assumes any legal liability or responsibility for the accuracy, com. pleteness or usefulness of any information, apparatus, product or process disclosed, or represents that its use would not infringe privately owned rights.

April 1, 1973 - March 31, 1974

PREPARED FOR THE U. S. ATOMIC ENERGY COMMISSION

UNDER CONTRACT NO. AT $(11-1)-3165$

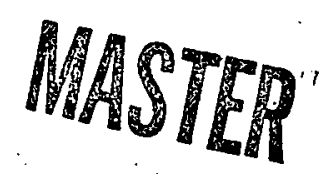

DISTRIBUTION OF. THIS DOCUMENT IS UNLIMITED 


\section{DISCLAIMER}

This report was prepared as an account of work sponsored by an agency of the United States Government. Neither the United States Government nor any agency Thereof, nor any of their employees, makes any warranty, express or implied, or assumes any legal liability or responsibility for the accuracy, completeness, or usefulness of any information, apparatus, product, or process disclosed, or represents that its use would not infringe privately owned rights. Reference herein to any specific commercial product, process, or service by trade name, trademark, manufacturer, or otherwise does not necessarily constitute or imply its endorsement, recommendation, or favoring by the United States Government or any agency thereof. The views and opinions of authors expressed herein do not necessarily state or reflect those of the United States Government or any agency thereof. 


\section{DISCLAIMER}

Portions of this document may be illegible in electronic image products. Images are produced from the best available original document. 


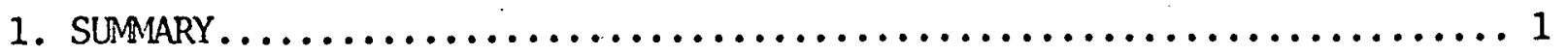

2. BRIEF CHRONOLOGY.................................... 2

3. NEW DEVELOPMENTS AND RESULTS $\ldots \ldots \ldots \ldots \ldots \ldots \ldots \ldots \ldots \ldots \ldots \ldots \ldots \ldots \ldots \ldots \ldots \ldots \ldots \ldots \ldots$

3.1 EXPERIMENTAL CONDITIONS AND MACHINE MODIFICATIONS $\ldots \ldots \ldots \ldots \ldots 5$

3.2 DIODE AND BEAM CHARACTERISTICS...................... 7

3.3 PLASMA HEATING................................. 8

3.4 ION ENERGY SPECTRUM..................................

3.5 BEAM INTERACTION WITH TURBULENT PLASMA.................. 11

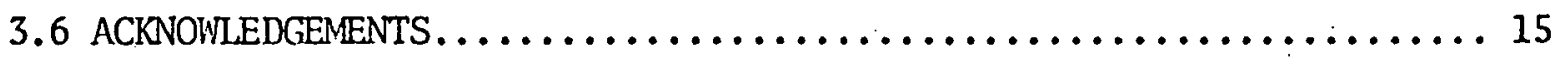

4. REFERENCES.......................................... 16

5. PUBLICATIONS AND CONFERENCE PAPERS PRESENTED..................16 6. APPENDICES

A. RELATIVISTIC ELECTRON BEAM HEATING OF A FULLY IONIZED PLASMA.

B. INTERACTION OF A RELATIVISTIC ELECTRON BEAM IITH A TURBULENT PLASMA.

C. INTERACTION OF A RELATIVISTIC ELECTRON BEAM AND A FULLY IONIZED PLASMA. 
1. SUMMARY

Several important new results were obtained during the year. These results, published in Appen. A, B and C, are summarized and discussed in this report. The experiments all involved rapid heating of a magneticallyconfined collisionless plasma colum with a high-current relativistic electron beam (REB). An important feature, which differentiates these experiments from some others, is that the fully-ionized plasma colum was injected through a $5 \mathrm{~cm}$ orifice along magnetic lines into a hard vacum in a $40 \mathrm{~cm}$ diameter chamber, eliminating the effects of walls and neutral gas around the column. Thus the electron beam did not create plasma, but only interacted with the already-existing, finite column.

Three distinct plasma conditions were used: (a) injected plasma having a temperature of tens of electron volts and density between $10^{13}$ and $6 \times 10^{13} \mathrm{~cm}^{-3}$; (b) Injected plasma heated to several $\mathrm{keV}$ by turbulent heating; and (c) highly turbulent plasma (electrostatic instabilities generated during turbulent heating). The strongest REB interaction was with plasma condition (c) (see Sec. 3.5 and Appen. B below).

Plasma heating to as much as $15 \mathrm{keV}$ per eleetron-ion pair was achieved. The ion energy distribution was enhanced at high energies, with the ions containing $1 / 3$ to $1 / 4$ of the total plasma energy. The plasma was stable after heating.

The brief Chronology has been updated to show our present and future undertakings.

Support for this program has been shared by AEC Contract AT(11-1) 3165 and ONR Contract N00014-67-A-0077-0022. 
2. BRIEF OHRONOLOGY OF CORNELL TURBULENT HEATING EXPEPINENT

Jan., 1968 Vacum chamber and magnet coils installed

Mar., 1968 First plasma

June, 1968 . Turbulent heating observed

Jan., 1969 Measured turbulence spectrum with microvave scattering; related spectral intensity to anomalous resistance and ion heating. Identified ion acoustic waves as heating agent. Electron density $\approx 10^{13}, W \approx 1$ joule, $\tau \approx 50$ usec.

Oct., 1969 Refined measurements of anomalous resistance and turbulence spectrum, using conductivity probe. Comparison with theory begun.

Jan., 1970 Modified plasma guns: $n_{e} \approx 5 \times 10^{13}$. Began measurement of spectrum of charge-exchange neutrals. Increased turbulent heating voltage from $\pm 18 \mathrm{KV}$ to $\pm 30 \mathrm{KV} . \mathrm{W} \approx 3.5$ joules.

May, 1970 Modified turbulent heating bank: new capacitors, new spark gaps, new d.c. supply, $\pm 100 \mathrm{KV}$.

Sept., 1970 Obtained consistent operation at $\pm 100 \mathrm{KV} . \mathrm{n}_{\mathrm{e}} \approx 10^{14}$ $W_{\mathcal{L}} \approx$ joules, $\tau \approx 150$ microseconds. Comparison to theory developed under sponsorship of AT (30-1) 3782.

Dec., 1970 Ion energy measurement, obtained by charge-exchange neutral analysis across and along the magnetic field. Results indicate non-Maxwellian ion energy distribution.

Aug., 1971 Completed first joint experiment with University of Texas team on ion heating at harmonics of ion cyclotron frequency. Found evidence that ICR heating also provided enhanced mirror containment, accompanied by a sloshing of plasma at the mirror bounce frequency. 
Oct., 1971 Parameter study using ion energy analyzer showed that ion energy depends on maximum electron drift velocity. Increasing $V$. increases ion temperature. Heating nearly independent of $\mathrm{B}_{\mathrm{Z}}$.

Jan., 1972 Second joint experiment with Texas team: damped oscillations from LC switched circuit impressed.

Feb., 1972 Electromagnetic wave scattering from turbulence at $74 \mathrm{GHIz}$. June, 1972 Third joint experiment with Texas team: I MN ion cyclotron wave heating. Found ion heating at $\omega_{\mathrm{ci}}$ and $2 \omega_{\mathrm{ci}}$.

Sept., 1972 Ion energy measurement, obtained by charge-exchange neutral analysis at five angles to the magnetic field. Results. show Maxwellian distribution drifting in ion frame at low energies, and enhanced high energy tail in direction of ion acoustic wave propagation.

Dec., 1972 Iristalled relativistic electron beam system (REBS) on end of TIM in place of one plasma source.

Jan., 1973 Testing of REBS begun. Beam injection in vacuum and singleended THM plasma.

Mar., 1973 Experiments begun on plasma heating by interaction of REB with THM plasma.

June, 1973 Preliminary experiments completed. Plasma heated to $W \geq 5 \times 10^{17} \mathrm{eV} / \mathrm{cm}^{3}$, ion energy non-Maxwellian, with higher average energy than for direct turbulent heating. Interaction of REB with turbulent plasma gave enhanced efficiency. 
July, 1973* Parameter studies of scaling lavs begun.

Jan., 1974\% Laser-scattering to measure electron temperature.

June, 1974** Injection of REB across B-field into plasma.

Sept., $1974^{* *}$ REB heating of $10^{15} \mathrm{~cm}^{-3}$ plasma.

*Experiments not yet completed. **Experiments proposed. 
3. NEW DEVELOPMENTS AND RESULTS

3.1 EXPERINENTAL CONDITIONS AND MACHINE MODIFICATIONS

The relativistic beam experiments were performed in the same apparatus as previous turbulent heating investigations*, permitting a direct comparison of results, using established diagnostics. The only modification was that the plasma source was removed from one end of the turbulent heating machine (THM), and the anode of the relativistic electron beam diode was installed to become one of the heating-current electrodes. Aside from a $25 \%$ decrease - in plasma density (the remaining plasma source was turned up somewhat), the operation of the single-ended machine was comparable to double-ended operation for direct turbulent heating.

Fig. 1 shows a schematic sketch of the modified installation. The Maxwell Pocobeam accelerator may be command-triggered, but the firing jitter in the self-firing mode was small enough to be acceptable for our experiments. Beam diode configurations are discussed in Sec. 3.2 and Appen. C. In most experiments the beam diameter was made the same as the plasma column diameter. Reasons for this choice are given in Sec. 3.2 below.

Parameters, of the modified REBS-THM machine are:

$$
\begin{aligned}
& \mathrm{V}_{\mathrm{b}} \text { up to: } 500 \mathrm{keV} \quad ; I_{\mathrm{b}}=5000 \text { to } 85,000 \text { amps } \\
& \beta=0.86 ; \gamma=1.96 \quad ; \mathrm{T}=60 \mathrm{nsec} \text {. } \\
& \mathrm{I}_{\text {LAWSON }}=17,000 \text { B } \gamma=28,500 \text { amps. } \\
& \frac{v}{\gamma}=\frac{I_{b}}{I_{L}} \leq \frac{85,000}{28,500}=3(\mathrm{MAX}) \\
& \text { Beam power } P_{b} \text { up to } 4 \times 10^{10} \text { watts } \\
& \text { Beam energy } W_{b} \text { up to } 2500 \text { joules } \\
& \text { Beam diameter }=\text { plasma diameter } \approx 5 \mathrm{~cm} \\
& \text { Magnetic field } \mathrm{B}_{Z}=7: 3: 6 \mathrm{kG} \text { mirror } \\
& \text { Plasma density } \mathrm{n}_{\mathrm{e}}=10^{13} \text { to } 6 \times 10^{13} \mathrm{~cm}^{-3}
\end{aligned}
$$

*See Annual Progress Report Coo-3165-3 (1972-73) . 


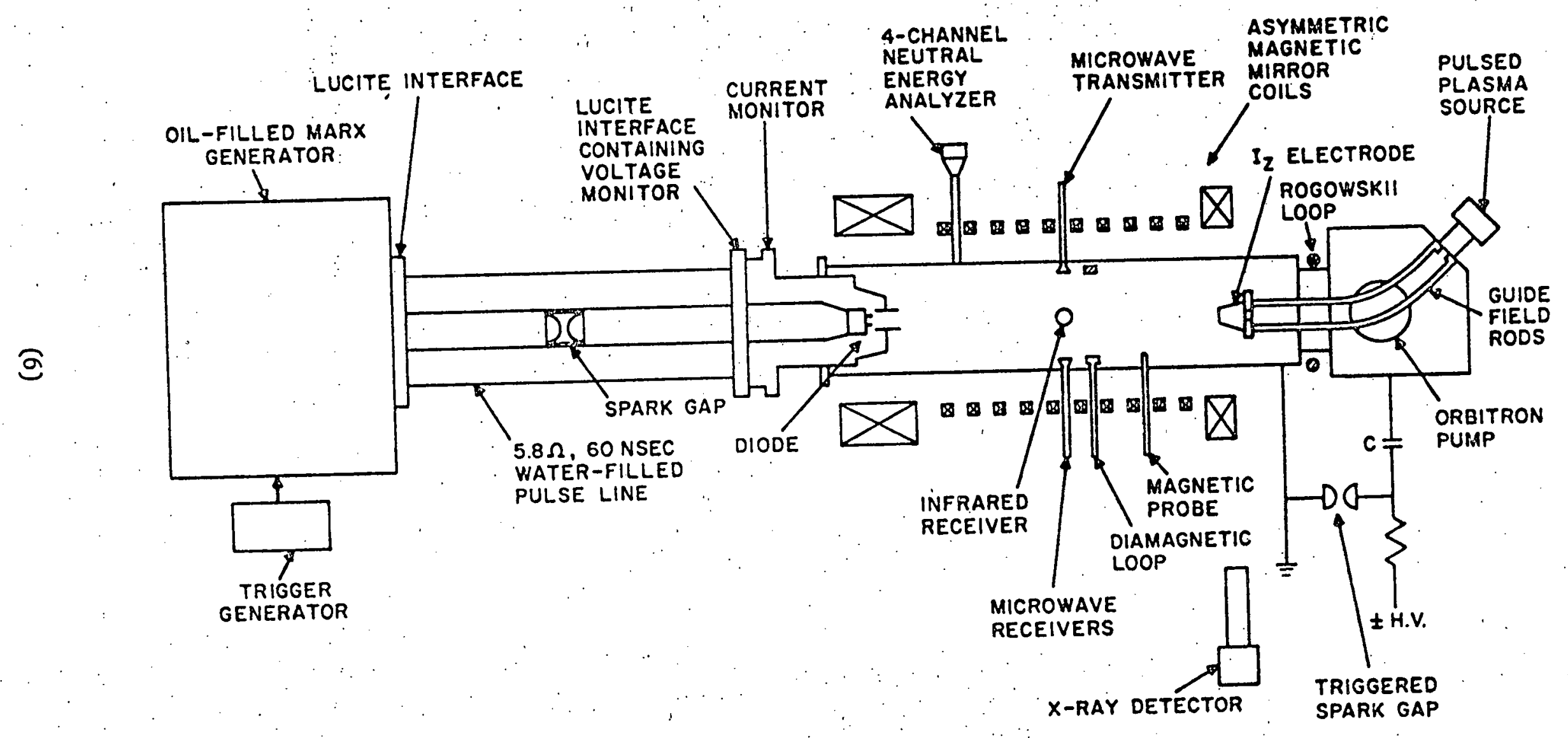

FIGURE 1. The Relativistic Beam and Turbulent Heating Machine. 


\subsection{DIODE AND BEM CHARACTERISTICS}

Two types of diode structures were used, one with a $2.54 \times 10^{-3} \mathrm{~cm}$ thick titanium anode foil, and one without. The plasma heating indicated by the diamagnetic loop and charge-exchange-neutral spectrum analyzer was similar for both configurations as long as plasma was allowed to enter the anode region. The main disadvantage of the foil anode was that at currents above $30 \mathrm{kA}$ the foil ruptured after 5 to 50 firings requiring that the machine be opened for anode replacement. That procedure causes delays and unavoidable vacuum contamination, leading us to adopt the foil-less diode for most high-current experiments. The beam diameter usually was matched to that of the plasma column, with the diode immersed in the magnetic field at the peak of the mirror.

Beam profiles were obtained by observing the damage patterns on $1 \mathrm{~mm}$ diameter plexiglass rods inserted at intervals along the path. When the beam was made smaller than the plasma diameter, it tended to expand during propagation until it just filled the plasma colum. Conversely, when a large diameter hollow beam was injected, it filled in during propagation to become solid, again occupying the same cross section as the plasma column. Thus the beam electrons were able to cross magnetic field lines, presumably due to some kind of instability or by turbulent diffusion. Further investigations of this important effect (important for $\mathrm{REB}$ injection into closed magnetic systems) are continuing and are part of the proposed work for next year.

The foil-less diode impedance was found to depend on several features, but was most sensitive to the presence of magnetized plasma in the anode region. Without magnetized plasma (to neutralize spacecharge) the impedance was high, and very little current could be extracted. The current that was extracted did not propagate very far in the vacuum magnetic field. 
Without magnetic field but with plasma the impedance was very low due to direct electron transit to the anode ring, followed by "gap closure" from metal vapors filling the anode-cathode space.

With fully-ionized plasma present, in a strong magnetic field ( $B \geq 5000$ gauss), a stainless-steel tubular anode and a carbon ring or tungsten-coated aluminum cathode were useful for $\sim 500$ firings, at impedances as low as 6 ohms. Considerable anode and cathode erosion occurred unless the proper geometry and conditions were found.

\subsection{PLASMA HEATING}

The apparatus was constructed to permit plasma heating by three methods: (a) with the relativistic electron beam, (b) by current-driven turbulent heating or (c) by both simultaneously. For direct turbulent heating we found that the onset-threshold was at a plasma current of $\sim 8000$ amperes. As the current exceeded that value there was a large increase in plasma resistivity and hot ions appeared. (1) In our experiment 8000 amperes creates an electron drift velocity $v_{D}$ approximately twice the ion sound speed $C_{S}$. This fact, along with other evidence, has led us to conclude that the ion acoustic instability is responsible for the plasma heating.

When the beam is injected alone, we found that the heating began as the beam current exceeded 8000 arperes. The heating can be explained also by the ion acoustic instability, where the electron drift is caused by the beam-induced reverse current. ${ }^{(2)}$ Figure 2 shows the suddent onset of heating and the steep dependence of total perpendicular energy $W_{\perp}$ on current. 


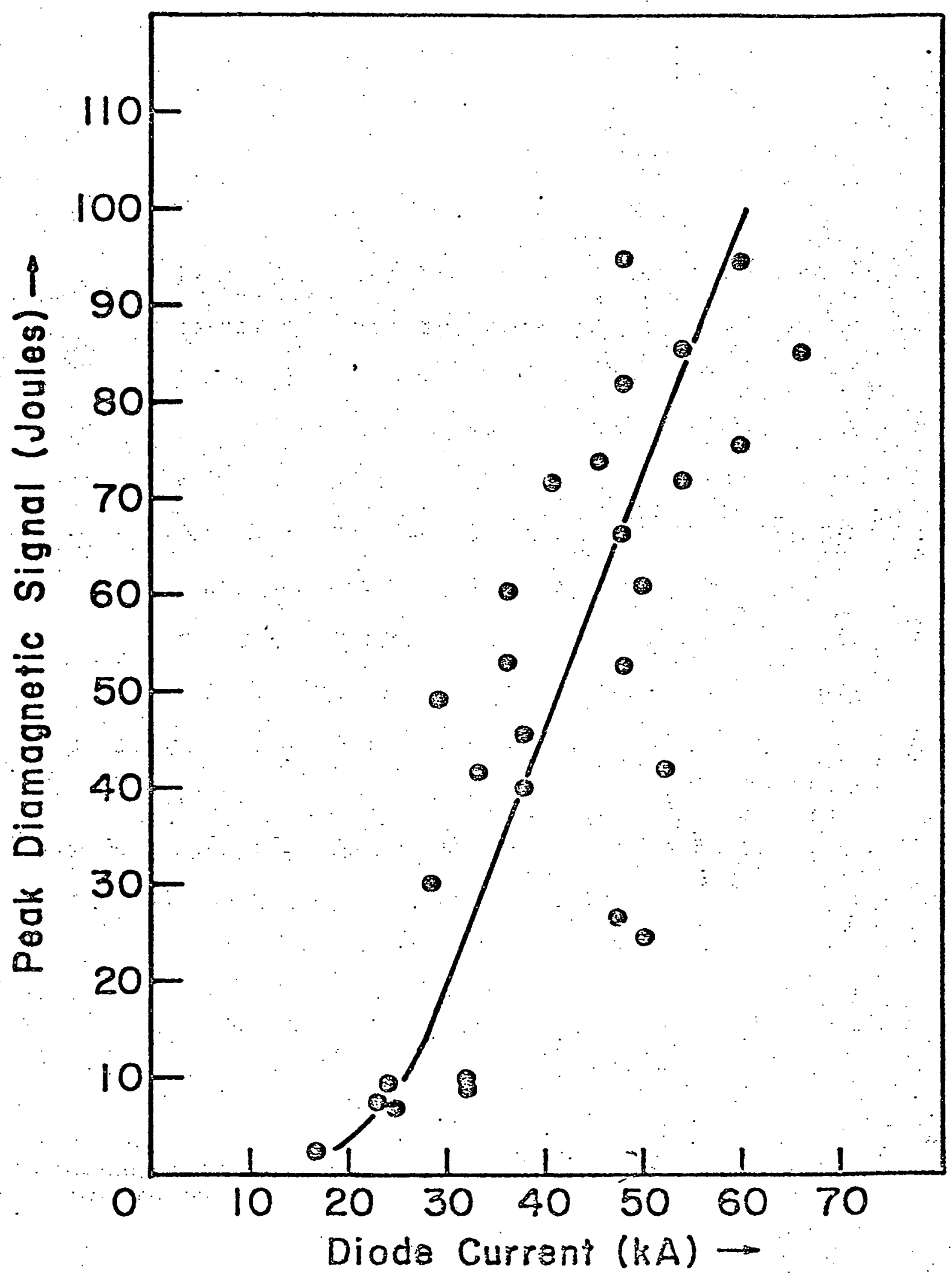

Fig. 2. Dependence of total perpendicular energy $W$, on diode current. Carbon cathode and foil-less anode used in diode. Magnetic field 3000 gauss at the diode. 
$W_{\perp}$ was obtained with a diamagnetic 1oop. The values shown were read just after the heating pulse, before the decay due to confinement losses. The time-dependence of the diamagnetic signal was very similar to that for direct turbulent heating. At the end of heating the maximum $W_{\perp}$ was about 100 joules, corresponding to an energy of $\geq 15 \mathrm{keV}$ per electron-ion pair. A rapid drop, with $\tau \sim 6$ sec, was followed by a slower decay with $\tau \sim 50$ to $100 \mu \mathrm{sec}$. We associate the initial decay with loss of electron energy (e.g. by thermal conductivity to electrodes) and the slower decay with loss of energetic ions by scattering and charge exchange. The decay time of the electron density, as read by a $4 \mathrm{~mm} \lambda$ microwave interferometer, was longer than either of the above. Occasionally, at high heating levels, there were rapid decay steps in the diamagnetic signal (3), followed by the usual slower decay. We have not yet determined the cause of these steps, but we presume that they are associated with some kind of instability, for example an electron velocity-space or cyclotron instability, expected for our conditions as the density falls. Accompanying the steps were bursts of $X$-rays and intense microwave radiation. The $4 \mathrm{~mm}$ microwave interferometer did not show a change in electron density coordinated with the steps.

A major disadvantage of the foil-less diode used in most of the above work is its sensitivity to plasma parameters. For example the diode impedance and thus the intensity of the relativistic electron beam are strongly dependent on the amount of plasma looking into the diode. Some plasma is needed to reduce the diode impedance to $\sim 6$ ohms but too much plasma causes diode closure. In order to avoid this coupling between the plasma and REB a diode having a foil anode was developed. At low currents $\left(I_{b}<40 \mathrm{kA}\right)$, the anode foil survived up to 100 shots 
without rupture, making rapid data collection possible. With this modification it is now possible to maintain fixed REB parameters while varying plasma properties. It has also been possible to operate in regions of plasma parameter space which were previously inaccessible because of the need for some plasma in the diode region to attain a low diode impedance in the foil-less diode. With the foil diode it is possible to adjust the diode impedance simply and permanently by adjusting the anode-cathode spacing.

The dependence of the beam-plasma interaction on plasma density (and thus $\mathrm{n}_{\mathrm{o}} / \mathrm{n}_{\mathrm{e}}$ ) has been determined using a foil diode to produce the REB. As shown in Fig. 3, the perpendicular energy per electron-ion pair increases with increasing beam to plasma density ratio, $n_{0} / n_{e}$, attaining a value of $15 \mathrm{keV}$ when the beam density equals that of the plasma.

\subsection{ION ENERGY SPECTRUM}

The 4-channel analyzer for energetic neutral atoms ${ }^{(4)}$ was placed $42 \mathrm{~cm}$ from the diode to receive atoms escaping perpendicular to the magnetic field. Spectra were obtained for conventional turbulent heating and for heating with the beam. From Fig. 4 we see that the average ion energy is approximately 3 times as large for "moderate" bean heating as for "moderate" turbulent heating. Both types of heating led to enhanced high energy components, with the beam heating having slightly higher efficiency. Further details are found in the Appendices.

\subsection{BEAM INTERACTION WITH TURBULENT PLASMA}

We expected an enhanced interaction when the electron beam was injected into the already-turbulent plasma, as discussed in our 1973-74 renewal proposal. We observed this enhancement. In the experiment it is possible to inject the beam into three completely different plasma conditions by simply changing the time of firing the sparkgap. Condition one (negative times in Fig. 5 is the unheated plasma colum, condition two is the 


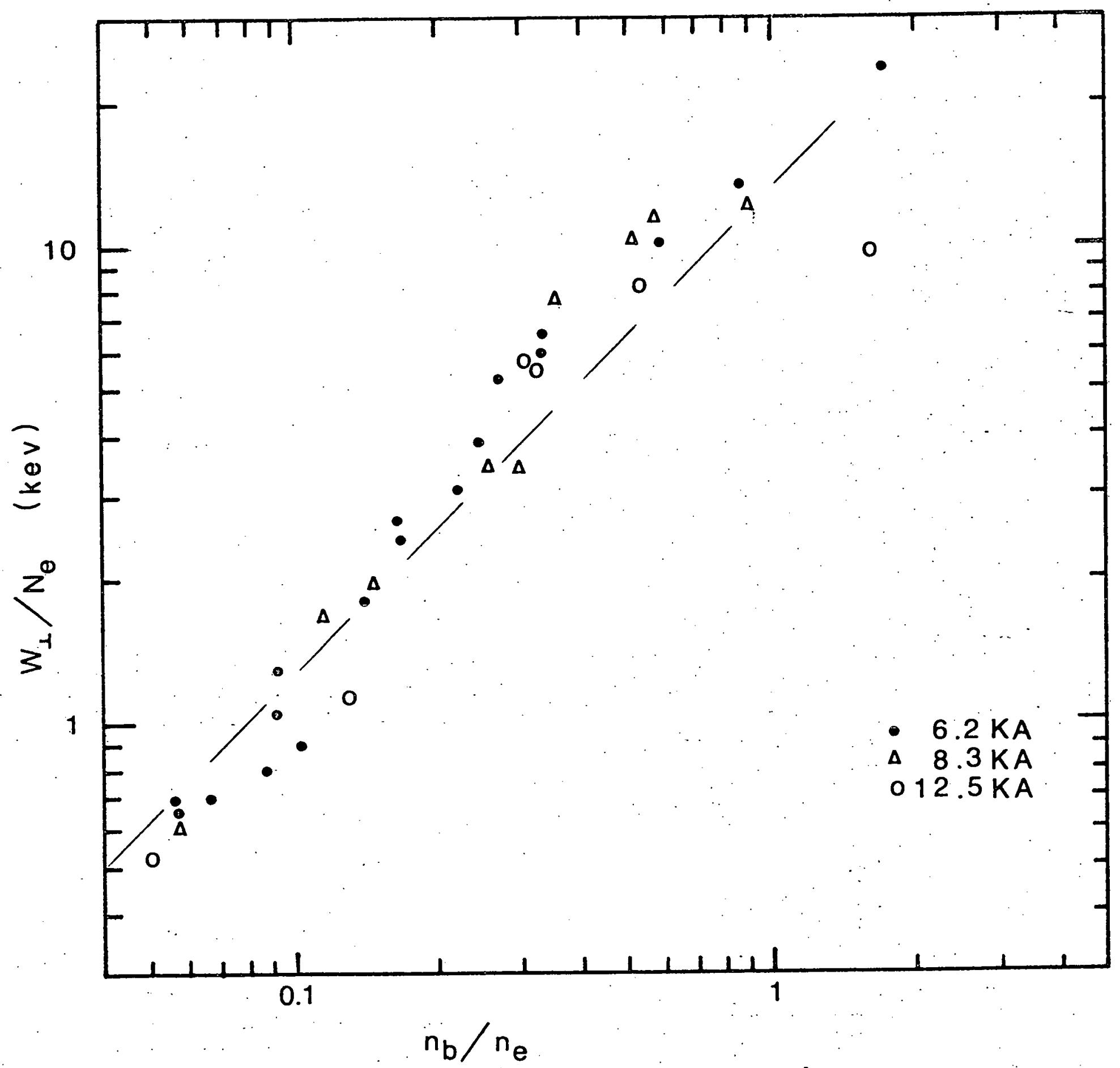

Fig. 3. Perpendicular energy per electron-ion pair as a function of the beam to plasma density ratio $\mathrm{n}_{\mathrm{o}} / \mathrm{ne}_{\mathrm{e}}$ for beam currents of $6.2,8.3$ and $12.5 \mathrm{kA}$. 


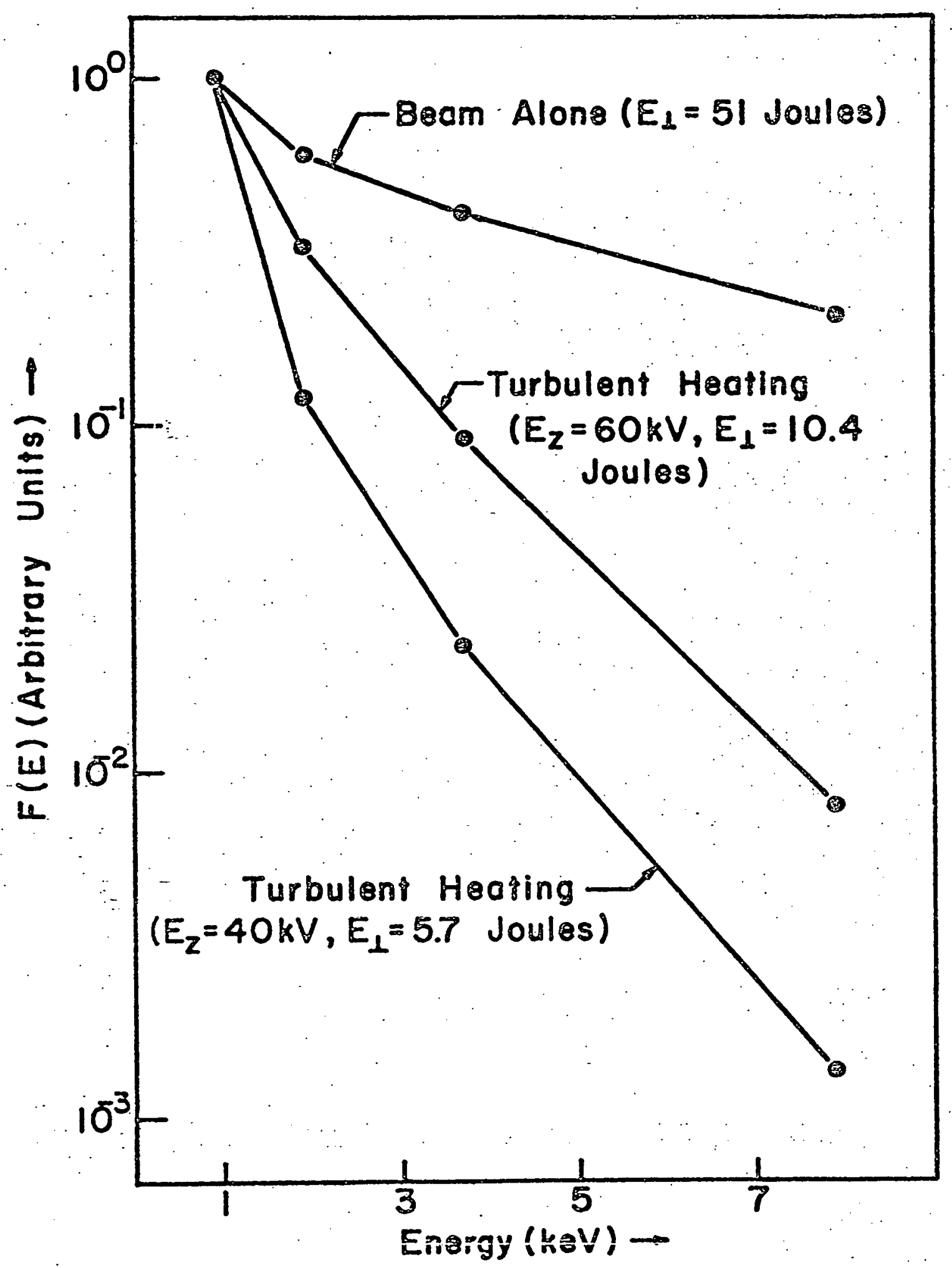

FIGURE 4:- Comparative Ion Energy Distributions for Beam Heating and Turbulent Heating. 


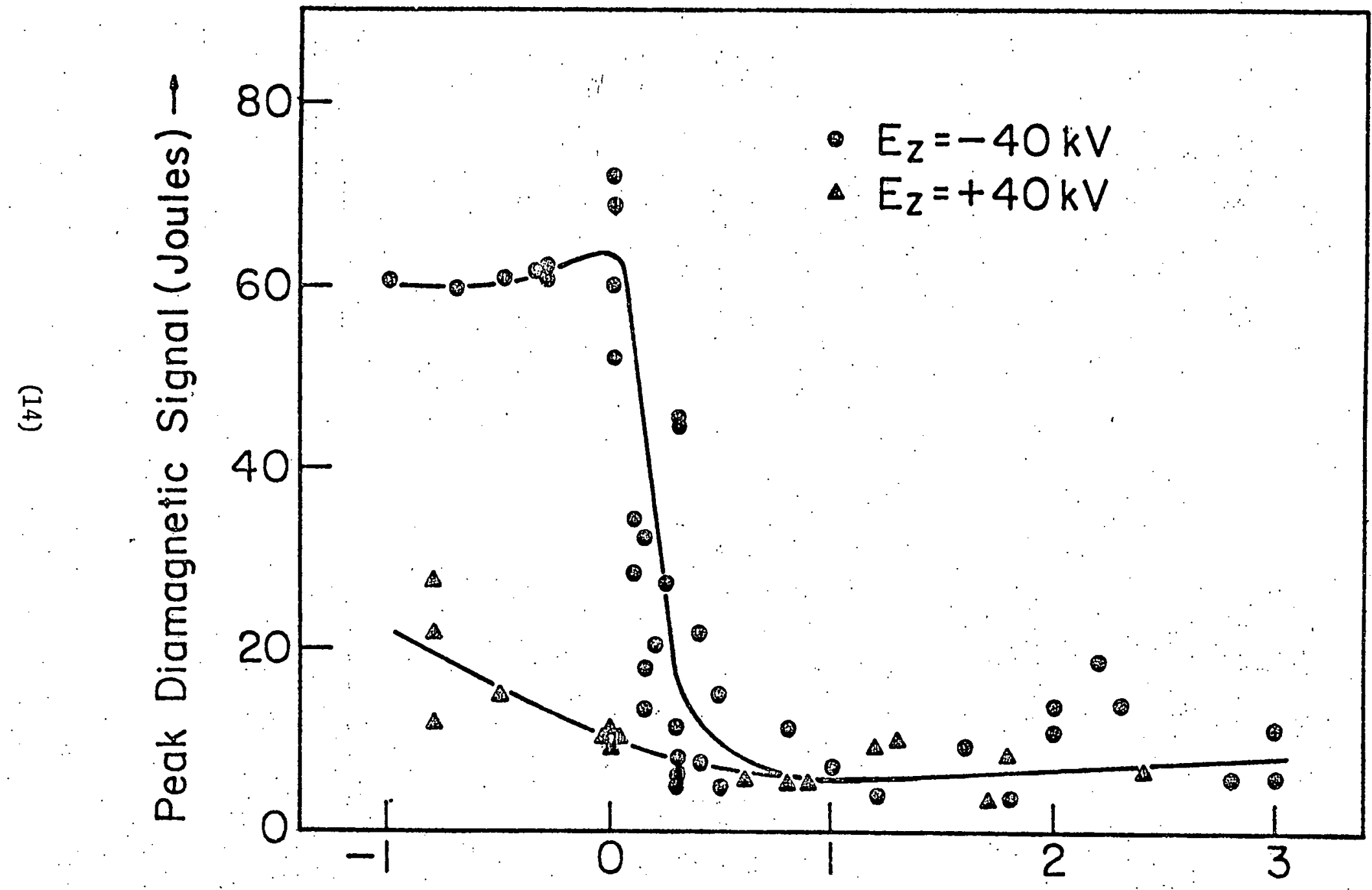

Delay Time Between Peak Turbulence and Beam Injection $(\mu \mathrm{sec}) \rightarrow$ FIGURE $5 \%$ Peak Diamagnetic Signal as a Function of Beam Injection Time Relative to Peak Plasma Turbulence. 
turbulent plasma colurn and condition three (positive times) is the quiescent, heated plasma colum having $C_{S}>V_{D}$. Fig. 5 shows $W_{\perp}$ indicated by the diamagnetic signal as a function of this time variation. The large heating during the time of anomalous resistance is evident. At low plasma densities where $C_{S}<V_{D}$ after turbulent heating, injection of the beam following $t=0$ gives enhanced heating, but as high densities there is little further heating after $t=0$.

The direction of turbulent-heating current may be reversed in respect to that of the beam-induced reverse-current. Fig. 5 also shows that when both currents flow in the same direction the heating enhancement is large. When they flow in opposite directions there is little enhancement. Refer to Appen. B for fuller details.

Using the foil diode it was also possible to investigate the effects of REB heating on the ordinary current driven turbulent heating. By : firing the REB first and pre-heating the plasma column by REB-plasma interactions, preverted the occurrence of current driven turbulence. This is an indication that the REB interaction increased the sound speed $C_{S}$ of the plasma so that the electron drift velocity $V_{D}$ never exceeded $C_{S}$ during the $I_{Z}$ current.pulse, thus no ion acoustic turbulence developed.

\subsection{ACKNOWLEDGEMENTS}

Major contributions were made by students M. Greenspan, F. Sandel and J. Sethian. Many technical contributions were made by P. Brown. 


\section{REFERENCES}

1. Robertson, S.; P. Korn and C. Wharton. IEEE Trans. on Plasma Sciences, PS-2 (2), 17 (1973).

2. Lovelace, R.V.E. and R.N. Sudan. Phys. Rev. Letters 27, 1256 (1971).

3. Korn, P., F. Sande1 and C. B. Wharton, Phys. Rev. Letters 31 (9), 579 (1973).

4. Greenspan, M.A. "Four-channel Neutral Particle Energy Analyzer " M.S. Thesis, Cornell University, (Aug. 1973) :

5. PUBLICATIONS AND CONFERENCE PAPERS PRESENTED

1. Korn, P., F. Sandel and C.B. Wharton, "Relativistic Electron Beam Heating of a Fully Ionized Plasma". Phys. Rev. Letters 31 (9) 579 (1973).

2. Korn, P., F. Sandel and C.B. Wharton, "Interaction of a Relativistic Electron Beam with a Turbulent Plasma". Submitted to Journal Applied Physics.

3. Korn, P., F. Sande1 and C.B. Wharton, "The Interaction of a Relativistic Electron Beam and a Fully Ionized Plasma". Submitted to IEEE Trans. on Nuclear and Plasma Sciences.

4. Greenspan, M.A., "Four Channel Neutral Particle Energy Analyzer". M.S. Thesis, Corne11 University (Aug. 1973).

5. Sandel, F. "HCN Laser Scattering System for Plasma Diagnostics". M.S. Thesis, Cornell University (1973).

6. Sandel, F., "The Interaction of a Relativistic Electron Beam and a Fully Ionized Plasma". Ph.D. Thesis, Cornell University (Aug. 1973). Also Bull. Amer. Phys. Soc. Ser. II, 18, 1350 (1973).

7. Korn, P., F. Sandel and C.B. Wharton, "Plasma Heating with a Relativistic Electron Beam". Proc. 6th Europ. Conf. on Contr. Fusion and Plasma Physics, P. 503 (Moscow. USSR 1973).

8. Kribel, R.E., C. Ekdah1, J. Sethian and C.B. Wharton, "Injection of a Relativistic Electron Beam into a Plasma". Bull. Amer. Phys. Soc. Ser. II, 18 (10) 1264 (1973). 
9. Johnson, M., R. Kribel, R.N. Sudan and C.B. Wharton, "Whistler Wave Studies". Bull. Amer. Phys. Soc. Ser. II, 181290 (1973). 
APPENDIX A.

PELATIVISTIC ELECTRON BEAN HEATING

OF A FULLY IONIZED PLASMA*

\section{by}

P. Korn, F. Sandel and C. B. Wharton

LPS 121

JUNE 1973

$\therefore$ This research was supported by the U. S. Atomic Energy Commission (AEC) (AT-11-1-3165iod/2) and the Office of Naval Research (ONR) (N00014-67-A-0077-0022). 
Relativistic Electron Bean Heating of a Fully Ionized Plasma*

P. Korn, F. Sandel and C. B. Wharton

Laboratory of Plasma Studies

Cornell University, Ithaca; New York 14850

\section{Relativistic electron beams with $.4<v / y<2.2$ have}

been used in this experisent to heat a high density

$\left(\mathrm{n} \sim 5-8 \times 10^{13} \mathrm{~cm}^{-3}\right)$ fully ionized hydrogen plasma immersed

in a magnetic field. The resulting heating levels are strongly

dependent on the effective diode impedance $Z=V / X$ and conres-

pondingly, the u/Y of the beam. Measurements of the total

plasma energy from the plasma diamagnetism and the ion energy

distribution from analyses ố charge exchange neutral atoms are

used to determine scaling lass for the beam-plasma interaction. 
Recent experiments ${ }^{1-4}$ using high energy relativistic electron beams have demonstrated the transfer of electrical energy into plasma thermal energy. The heating mechanism involves collective beam-plasma interactions, with two candidates being post likely: turbulent heating resulting from the beam return current $5,6,7(\nu / \gamma>1)$ and two-stream instabilities ${ }^{7,8}$ with $v / Y \sim 1$.

The Comell turbulent heating experiment 9,10 has been modified (Figure 1) to allow the introduction of a relativistic electron beam from a $5.8 \Omega$ Pocobeam ${ }^{11}$ accelerator capable of operating at $\leqslant 500 \mathrm{kV}$ with $\leq 86 \mathrm{kA}$ currents for a nominal 60 nsec pulse. A non-symetric magnetic mirror field is energized by a $120 \mathrm{~kJ}$ capacitor bank producing 5-6 kG at one mirror, $\sim 2.7 \mathrm{kG}$ in the mirror well, and $\sim 5 \mathrm{kG}$ at the plasmainjection end. A set of hexapole guide field rods conducts plasma from a pulsed hydrogen plasma source through the $(\sim 5 \mathrm{kG})$ mirror peak into the trap. Plasma density has been measured with a $74 \mathrm{CHz}$ fringe-sinift interferoneter and by the attenuation of a neutral atch bean where the microwaves are cut of ${ }^{9}$. The neutral background pressure is generally less than $4 \times 10^{-6}$. T except when charge-exchange neutral energy analysis is performed $P<2 \times 10^{-5} \mathrm{~T}$. In this paper only the direct beam interaction with the injected plasma will be considered.

In these experiments a foil-less diode, positioned behind the peak of the mirror field, consisting of a carbon cathode and a cylindrical anode has been used to obtain impedances as $10 \%$ as $6 \Omega$. This diode relies on the leakage of low density plasma from the wirror into the anode-cathode region to lower the effective diode impedance and guide the beam into the 


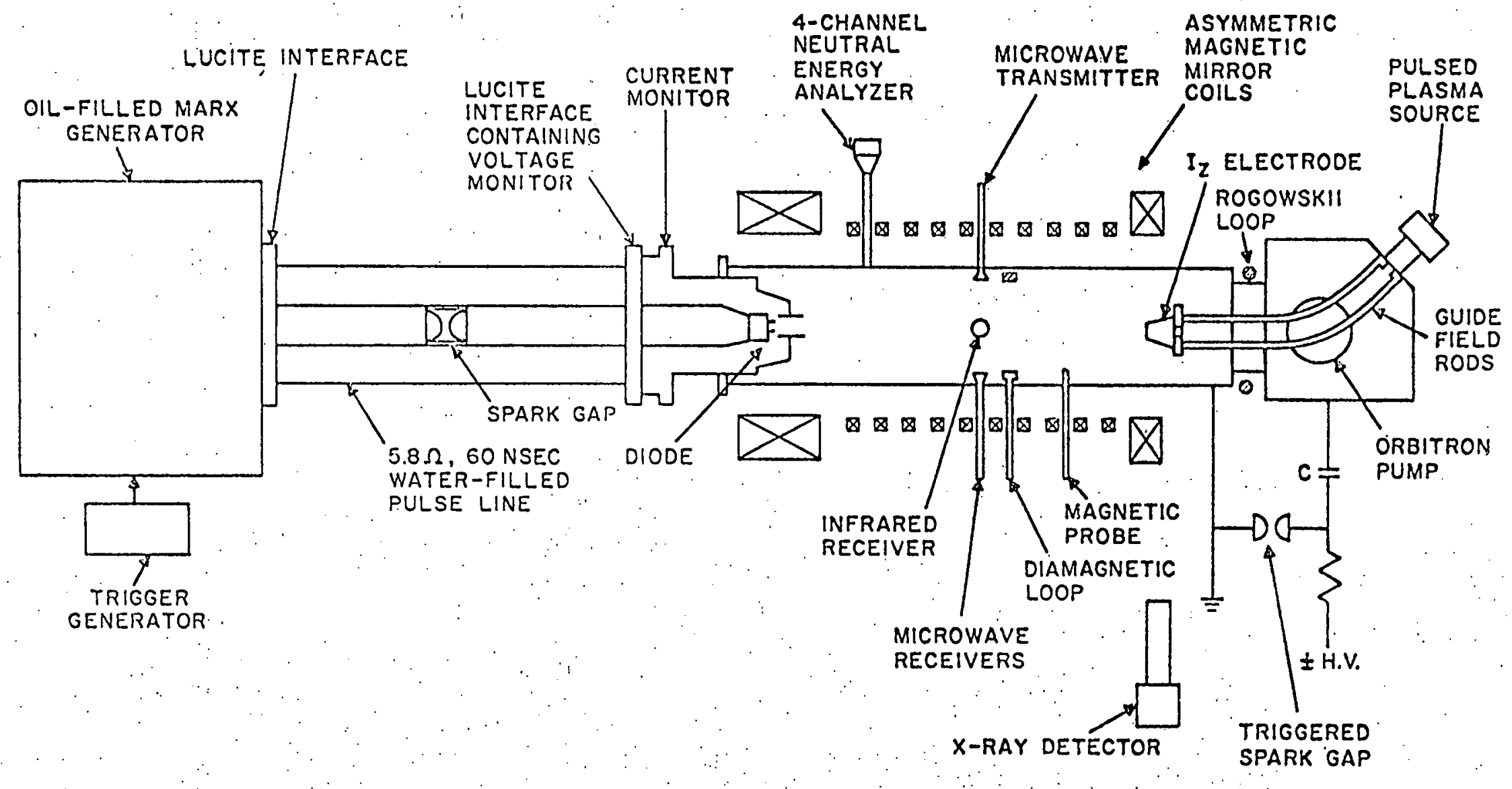

Figure 1. The Cornell turbulent heating - relativistic electron beam experiment. 
mirror. Anode structures with apertures of $2.5 \mathrm{~cm}$ and $5 \mathrm{~cm}$ have been used with appropriate carbon cathodes in this configuration.

Figure 2 indicates typical voltage; current, and plasma diamagnetism signals for electron beam heating. The presence of the injected fully ionized plasma in the diode region provides a stable impedance level with no evidence of diode closure for reasonable gap spacings $\sim 1 \mathrm{~cm}$. The diamagnetic signals displayed indicate the different time histories typical of various energy density levels. For diamagnetic signals less than 35 joules, there was generally a slo: decay characterized by a single rime constant. Between 35 and 60 joules, small discontinuities in the diamagnetic signal appeared. These were observed at various times following the rise of the signal, from a few microseconds to over 100 microseconds. They are attributed to sudden loss of energetic electrons. through an instability, and are usually associated vith bursts of hard $x$-rays from the plasma. Finally, at the highest heating levels observed. $(60-100 J)$, the dianagnetic signal typically shows a rapid decay fron its peak value followed by a transition to a much slorer decay. This is interpreted as preferential electron heating at these levels, a view supported by charge-exchange neutral data discussed below. The microwave interferometer does not indicate any significant increase in density during the first few wicroseconds following heating. On a longer time scale (10-200 Hsec) the plasma density increases substantially as cold plasma and neutral gas from the plasma source enters the mirror region and is heated or ionized by impact with the confined hot plasia. Experiments ${ }^{12}$ with a foiled diode at Io: currents determined a threshold for heating occurring at $\sim 8-10 \mathrm{kA}$. of diode current. This is nominally the level at which direct turbulent heating of the plasma occurs, suggesting that the same instabilities may be responsible for both types of heating at this level. 


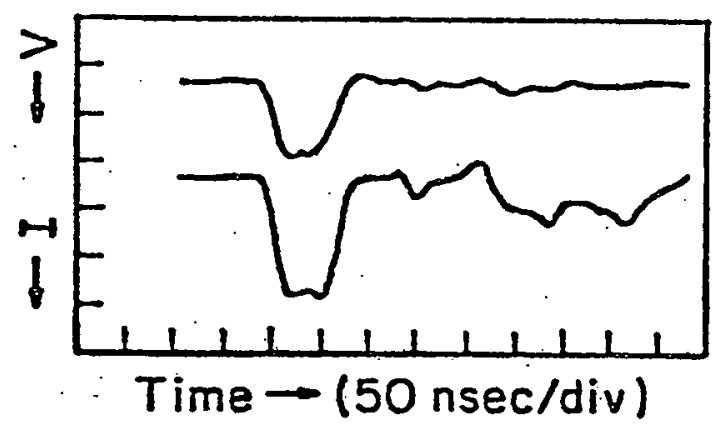

(a)

Vertical Scales

Voltage: $V \rightarrow(275 \mathrm{kV} /$ div $)$

Current: $I \rightarrow(20.8 \mathrm{kA} /$ div $)$

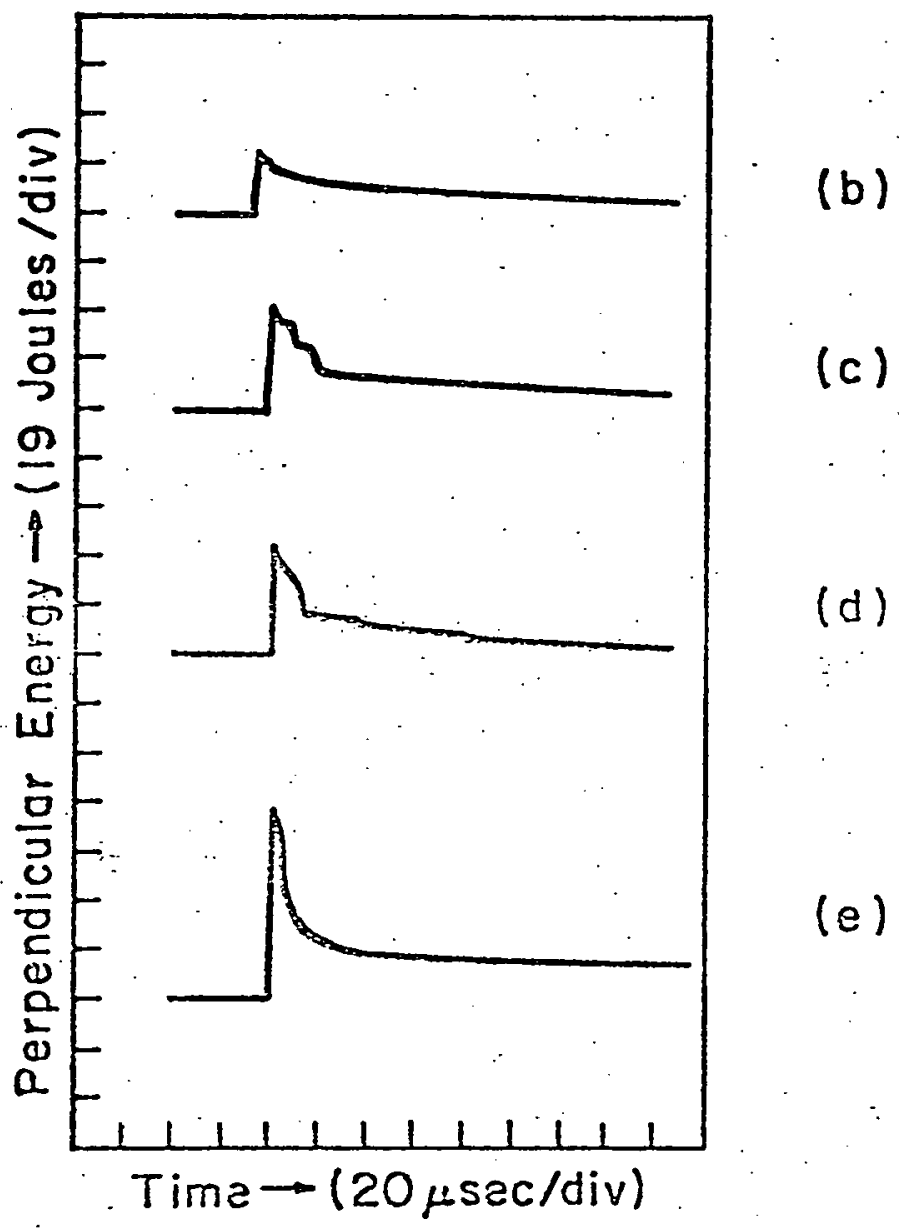

Figure 2. Oscilloscope traces for relativistic electron bean heating (a) diode voltage and current, (b) plasma diamagnetism, $E_{\perp}<35$ joules, $(c-d)$ plasna diamagnetism $E_{\perp}$ 35-60. joules, (e) plasma diamagnetism, $E_{\perp}>60$ joules. 
Figure 3 indicates the peak diamagnetic energy and the relative transfer efficiency ceasured for various diode impedance levels using a $5 \mathrm{~cm}$ foil-less diode. These data vere generated by adjusting both the beam firing time and the voltage level of the Marx generator since diode impedance is a very sensitive function of the timing between the plasma injection and beam firing. It is clear that for this type of direct beam-plasma interaction a low impedance (high $\nu / Y$ ) beam provided the highest energy transfer in the experiments reported.

In a separate experiment the mirror field peak at the electron beam diode was removed so that the field was increasing axially the entire length of the plasma column from the diode to the peak of the opposite mirror. Diamagnetic signals (rominally $-30 \mathrm{~J}$ ) had a characteristic life time of - I.5 - 2 usec corresponding to plasma diffusion out one end of the machine as compared to traces in Figure 2. The diamagnetlc loop is electrostatically shielded with a metal foil and has a response time of $\sim 1 / 2$ usec. This is taken as evidence that the diamagnetic signals are not due to trapped bean primary electrons as the characteristic decay time without a mirror would be much shorter than the observed times.

Observation of the radial profile of the electron beam by insertion of slender $.3-.6 \mathrm{~cm}$ damage rods indicated that the bear was contained in the $\sim 4 \mathrm{~cm}$ diameter of the injected plasma column $-30 \mathrm{~cm}$ from the diode. At this axial position the magnetic field had decreased from $\sim 5.5 \mathrm{kG}$ at the mirror peak to $3.6 \mathrm{kG}$. Possible fluting at tine periphery of the beam was noted. "At an axial position of $\sim 67 \mathrm{~cm}(\sim 2.7 \mathrm{kG})$ from the diode ine bean usually had either disappared or produced a diffuse vea' damage 


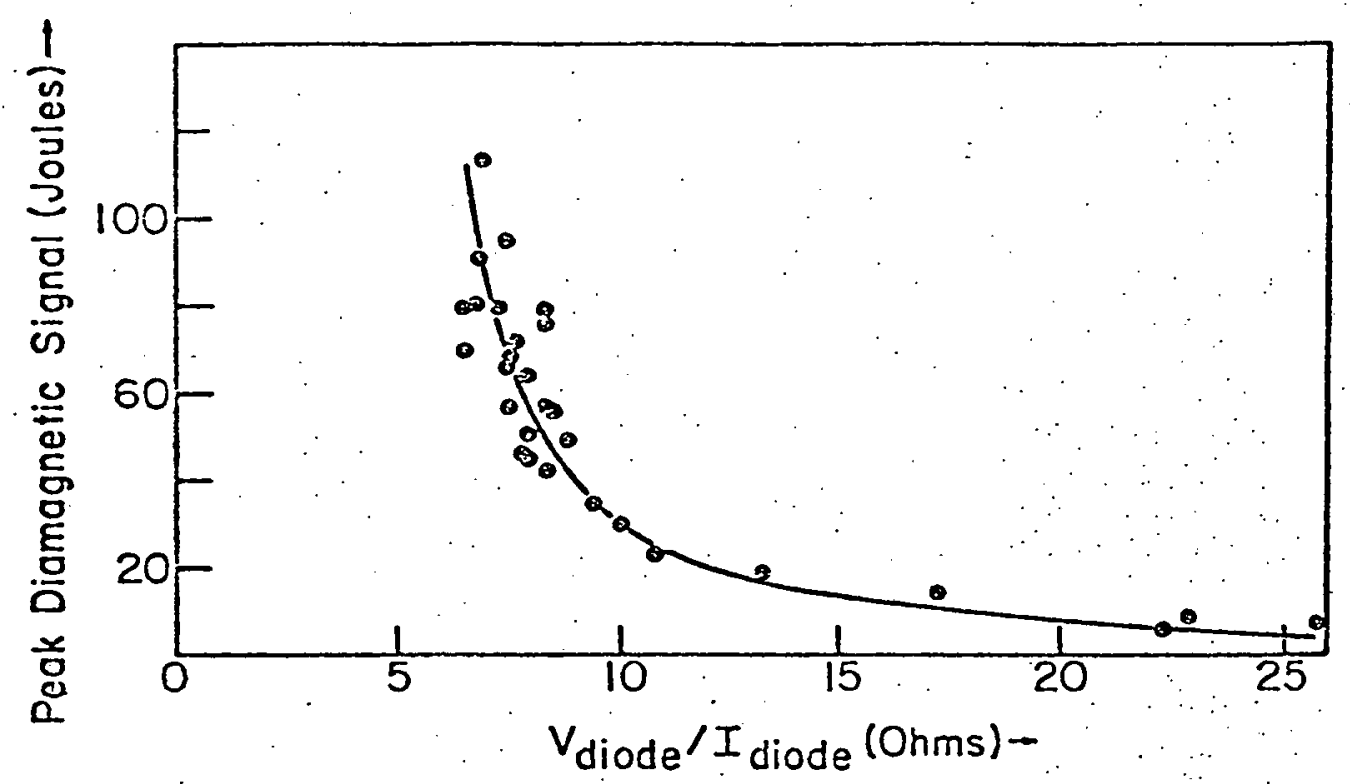

(a)

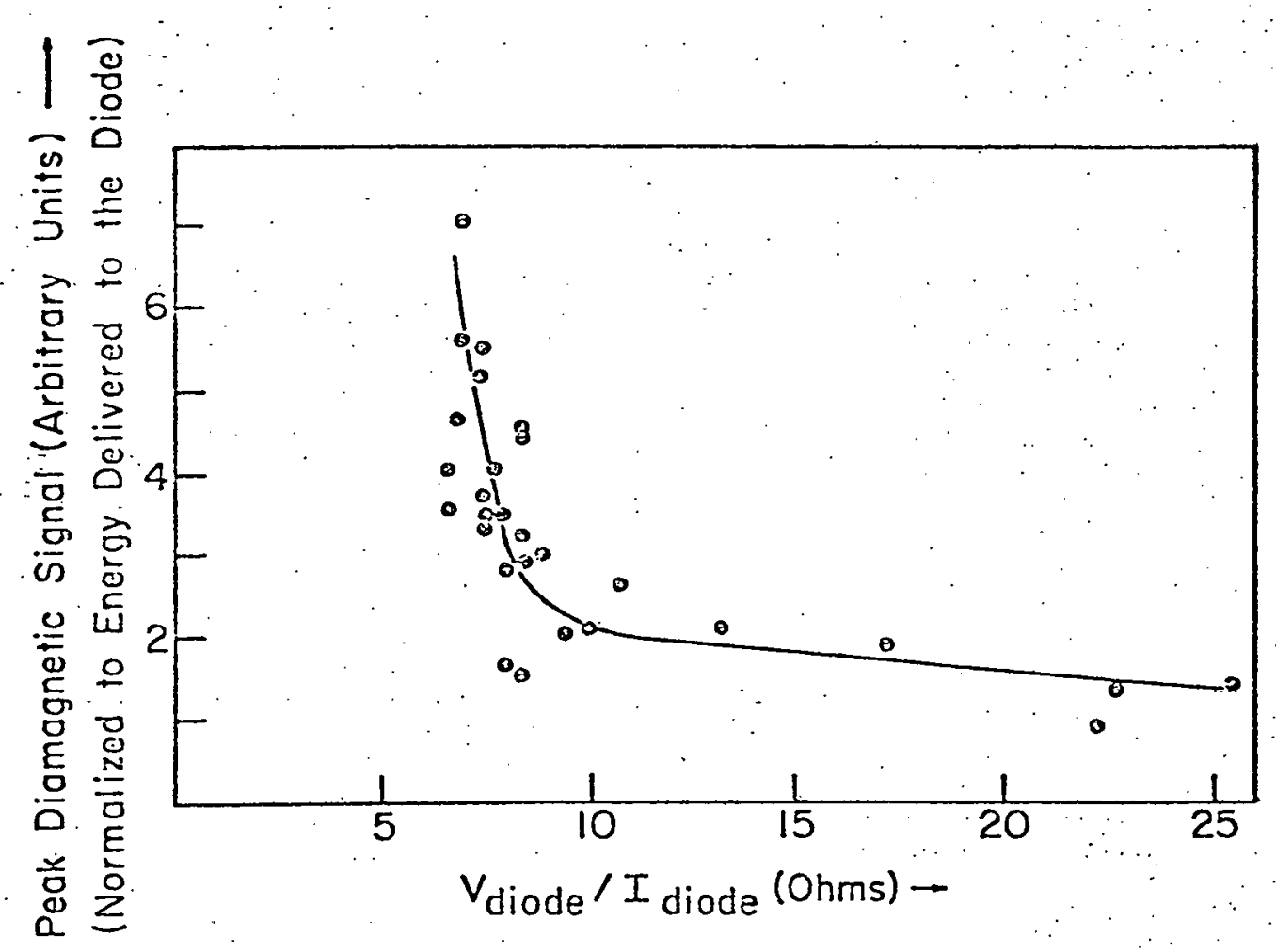

(b)

Figure 3. Peak plasma diamagnetism (a) and relative transfer efficiency (b) for relativistic electron beam heating using a 5 cri aperture foil-less diode. Fach point corresponds to one machine firing. Peali transfer efficiencies from input energy into the diode to perpandicular plasma energy $-10-15 \%$ 
pattern $\left(5-10^{\circ} \mathrm{cm}\right)$. The use of even thin danage rods obstructed the flow of plasma into the diode region, changed the diode impedance and consequently the experimental conditions. This precluded the use of Faraday cups or: calorimetry to determine bean parameters. Such behavior suggests that the beam is guided into the mirror by the injected plasma column which provides magnetic and electric neutralization for the beam. As the beam moves from the peak mirror field towards the well the energy density of the electron beam becomes comparable to the magnetic field energy density wich is probably an undesirable feature for beam propagation. ${ }^{13}$ when one mirror was removed, as discussed previcusly, damage rods indicated that although the bean was weakened considerably it did not blow up. Figure $4 \mathrm{a}$ shows the plasma diamagnetic energy contained in the mirror following the beam heating for various diode currents during a single run. For this configuration our line density $N-1.5-2 \times 10^{14} \mathrm{~cm}^{-1}$ corresponds to a mean perpendlcular energy per electron-ion pair $\geq 5 \mathrm{keV}$ for 30 joules in the mirror. To obtain these data the firing time of the electron bean relative to the time of plasma injection was held constant and the energy in the beam varied by charging the accelerator to different voltages. Each point corresponds to one machine firing. Data of the Eype displayed in Figures 3 and 4 were obtained for both 2.5 and 5 cm Eoilless diodes and data obtained with anode foils at low currents ( $\leq 25$ kA) is entirely compatible. In Figure 3 beam voltages vere in the range 250 - $500 \mathrm{kV}$ corresponding to a maximum Alfvén current of $\sim 28.5 \mathrm{kA}$. It. is evident that this is the approximate threshold region for strong beam heating which in this experiment corresponds to the condition $v / \gamma>1$. 

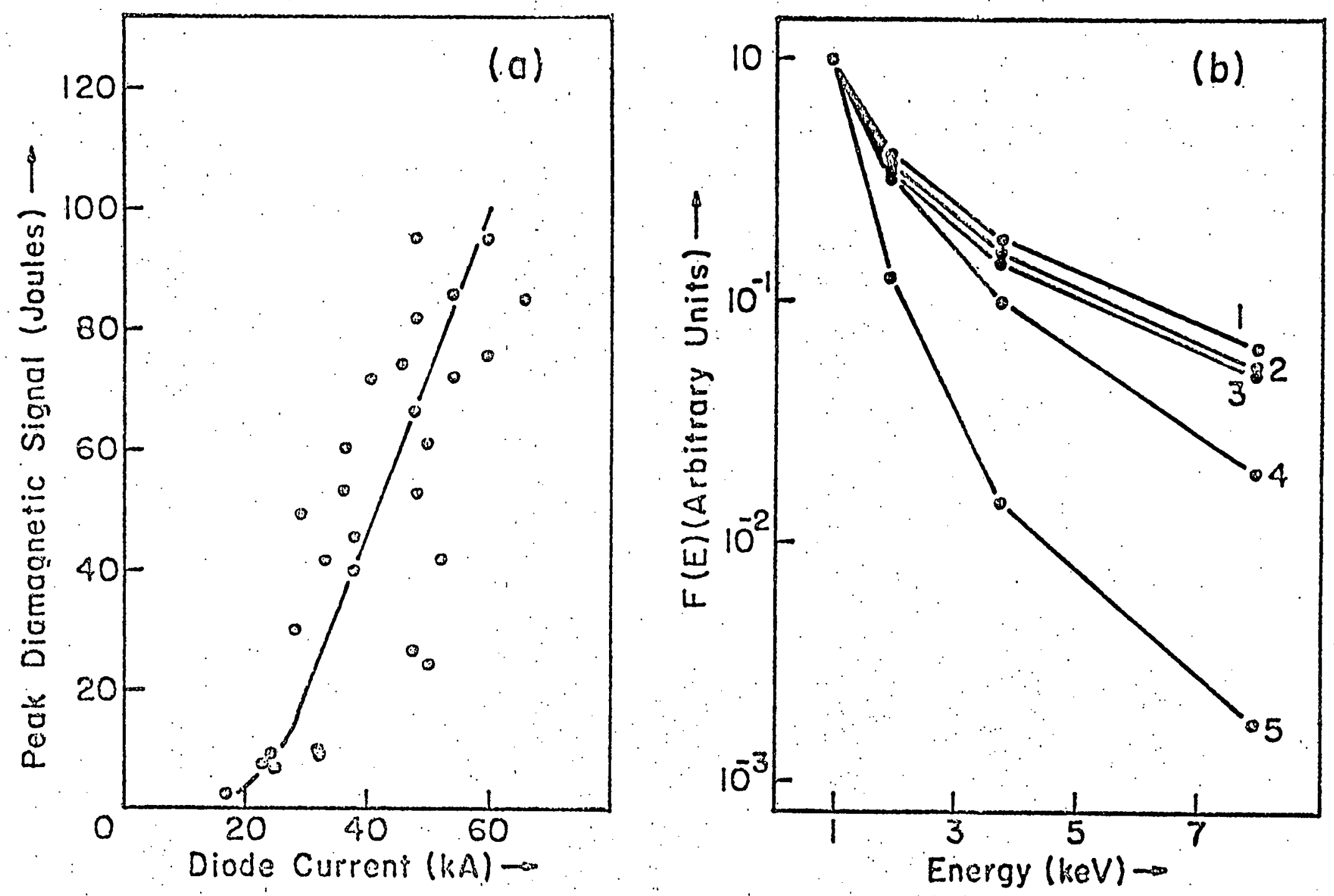

Figure" 4a. Perpendicular plasma energy vs. diode current for a fixed beam-plasma Injection time le $\sim$ constant impedance diode using a $2.5 \mathrm{~cm}$ aperture foll-less diode.

Figure 4b. Ion distribution function vs. energy for various beam heating levels;

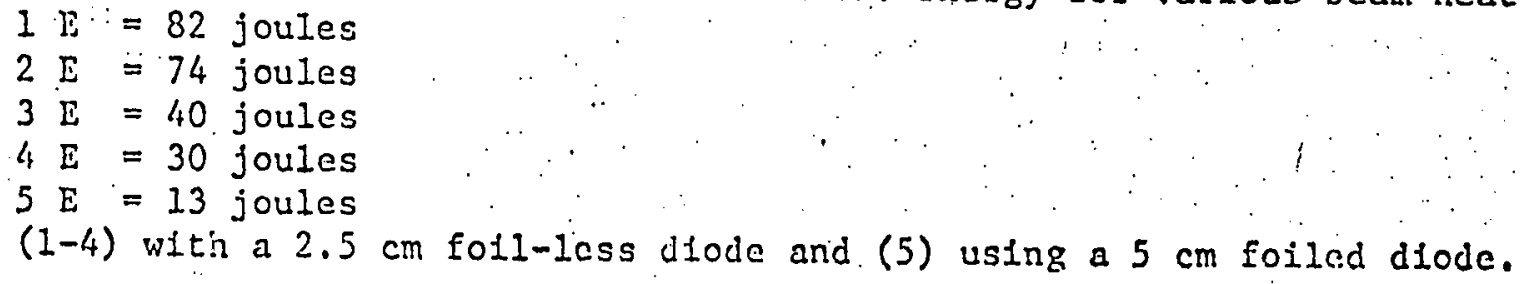


Figure $4 \mathrm{~b}$ presents data acquired using a four chamel neutral energy analyzer $-42 \mathrm{~cm}$ from the diode. Each curve corresponds to the data from one machine firing and the curves labeled $1-4$ are representative shots from Figure 4. Curve 5 is shown for comparison to indicate appropriate scaling of the ion energy spectrum at low diamagnetic energies. These data were normalized at $925 \mathrm{eV}$ because the absolute number of neutral atoms resulting from charge exchange is a sensitive function of the neutral kryption pressure, plasma density, energy and expansion velocity and therefore only relative levels are important."

An examination of the ion distribution indicates that at 10is energies $\left(E_{\perp} \leq 40\right.$ joules) changes in diamagnetic signal result in large changes in the ion distribution, while above this level the relative changes in the ion spectrum are a slower function of $E_{\perp^{-}}$. This may be indicative of preferential electron heating above average energies of $\sim 6 \mathrm{keV}$ or may result from the loss of confinement of ions during the heating process because of the low magnetic field $-3 \mathrm{kG}$ at axial positions in front of the analyzer: Neutral data taken at an axial position $-120 \mathrm{~cm}$ frow the diode in a ragion well past the axial extent of the electron beam indicated slowly rising neutral signals. This results from ion heating occurring near the diode region vith subsequent diffusion of hot plasma into the aperture of the analyzer followad by charge exchange: This is consistent with observations of electron beam behavior with danage rods:

From the experizental results one concludes that relativistic electron beams can be used to heat fully ionized plasmas to energy densities of interest for Controlled fusion. These plasmas appear to be stably confined immediately following neating, and it is possible to reliably transfer $10-15$ percent of the electrical energy supplied to the diode into plasma perpendicular energy. 
In order to ensure maximum energy transfer it is necessary to have a sufficiently large magnetic field to suppress beam destruction via instabilities. Electron beans with $\nu / Y>1$ appear to be most desirable for efficient plasma heating suggesting that the return current driven instabilities may be the most promising for plasma heating as predicted by Lovelace and Sudan. 5

The authors wish to thank Prof. H. Postoker and Prof. R. Sudan for nany valuable discussions, and Dr. S. H. Robertson and Mr. M. A. Greenspan for assistance with the neutral aton energy analyzer.

* Research supported in part by the U. S. Atomic Energy Conmission and the Office of Naval Research. 


\section{References}

1. A. T. Altyntsev et al., Pis'ma Zi. E:ssp. Teor. Fiz. 13, 197 (1971) [JETP Lett. 13139 (1971)], and in Proceedings of the Fourth International Conference on Plasma Physics and Controlled Nuclear Fusion Research, Madison, Wisconsin, 1971 (International Atomic Energy Agency, Vienna, 1972), Vol. 2 p. 309.

2. C. A. Kepetanakos and D. A. Hawer to $b \geq$ published in Applied Physics Letters July 1, 1973, and C. A. Kapatanakos et al., Bull. Amer. Phys. Soc. 17,1032 (1972).

3. G. C. Goldenbaum et al., Bull. Aner. Phys. Soc. 17, 1031 (1972).

4. C. Stallings et al., Bull. Amer. Phys. Soc. 17, 1031 (1972).

5. R. V. Lorelace and R. N. Sudan, Eiys. Rev. Leti. 27, 1255 (1971).

6. J. Guillory and G. Benford, Plassa Physics 14, 1131 (1972).

7. A. A. Ivanov and L. I. Rudakov, Sov. Phys. JETP 31, 715 (1970).

8. L. E. Thode and R. N. Sudan, Phys. Rev. Lett. 30, 732 (1973).

9. S. Robertsoi, P. Korn and C. B. Wharton to appear in Transactions IEE Soc. of Nivclear and Plasma Seiences. 
10. C. Wharton et al., Proceedings of the Fourth International Conference on Plasma Physics and Controlled Nuclear Fusion Research, Madison, Wisconsin, 1971 (International Atomic Energy Agency, Vienna, 1972), Vol. 2 p. 25 .

11. Manufactured by Maxwell Laboratories, Inc.

12. P. Korn, F. Sandel and C. B. Wharton to appear in Proceedings Sixth European Conference on Controlled Fusion and Plasma Physics, Moscow, USSR, July 30 - Aug. 3, 1973.

13. R. N: Sudan, Bul1. Amer. Phys. Soc. 18, 585 (1973). 
APPENDIX B.

INTERACTION OF A RELATIVISTIC ELECTRON

BEAM WITE A TURBULENT PLASMA*

by

P. Korn, F. Sandel, and C. B. Wharton

LPS 124

JUNE 1973

* This work was supported jy the U. S. Atomic Energy Comnission (AEC) under contract number (AT-11-1-3165ilodit2) and the office of haval Research (O:R) unier contract number (N00014-67-A-0077-0022). 


\section{Interaction of a Relativistic Electron Beam with a Turbulent Plasma* \\ P. Korn, F. Sandel, and C. B. Wharton Laboratory of Plasma Studies Cornell University, Ithaca, New York 14850}

Abstract

The interaction of a relativistic electron beam $(v / y-2)$ with the turbulen: phase of a z-discharge is examined. An enhancenent in heating is observed then the electron current due to the $Z$-discharge is in the opposite direction to the beam current and the plasma is highly. turbulent. The ion energy distribution is examined for turbulent heating, beam heasing, and combined beam and turbulent heating. 
Previous experiments at Corne $11^{1,2}$ and elsewhere ${ }^{3-6}$ have investigated the effectiveness of relativistic electron beams for. plasma heating. In this work a relativistic electron beam has been injected into a turbulent $Z$-discharge in order to study the interaction. In a sense the injection of a relativistic electron beam may be regarded as an alternative technique for supplying a current to a plasma. Many of the same basic instabilities ${ }^{7-10}$ that transfer energy to the plasma may be expected.

The Cornell turbulent heating experiment ${ }^{11,12}$ as modified (Figure 1 ) to accommodate a $500 \mathrm{kV}, 5.8 \Omega$ Pocobeam ${ }^{13}$ accelerator has been described previously. ${ }^{1,2}$ A high density (n $-5-8 \times 10^{13} \mathrm{cI}^{-3}$ ) fully-ionized. hydrogen plasma is injected into a vacuum region in a non-symmetric. magnetic mirror which provides up to $5-6 \mathrm{kG}$ at the diode with $2.7 \mathrm{kG}$ in the mirror well and $5 \mathrm{kG}$ at the opposite mirror where the plasma is injected. Turbulent heating is achievad oy applyiag $40-80 \mathrm{kV} \cdot$ to the electrode terminating the hexapole guide field rods and passing $\leq 30 \mathrm{kA}$ through the nominal. 2 cm, radius plasma column.

The electron bean, injected axially into the plasma column, can be. comand-fired with 10: jitter (standard deviation of $~-60$ nsec) with respect to the discharge of the turbulent heating capacitor bank by triggering the Narx generator and allowing a pressurized $S F_{6}$ spark gap in the vater. pulse line to overvoliage.

Single-ended operation of the turbulent heating machine (one plasma source and non-symmetric voltage application) is qualitatively the same 


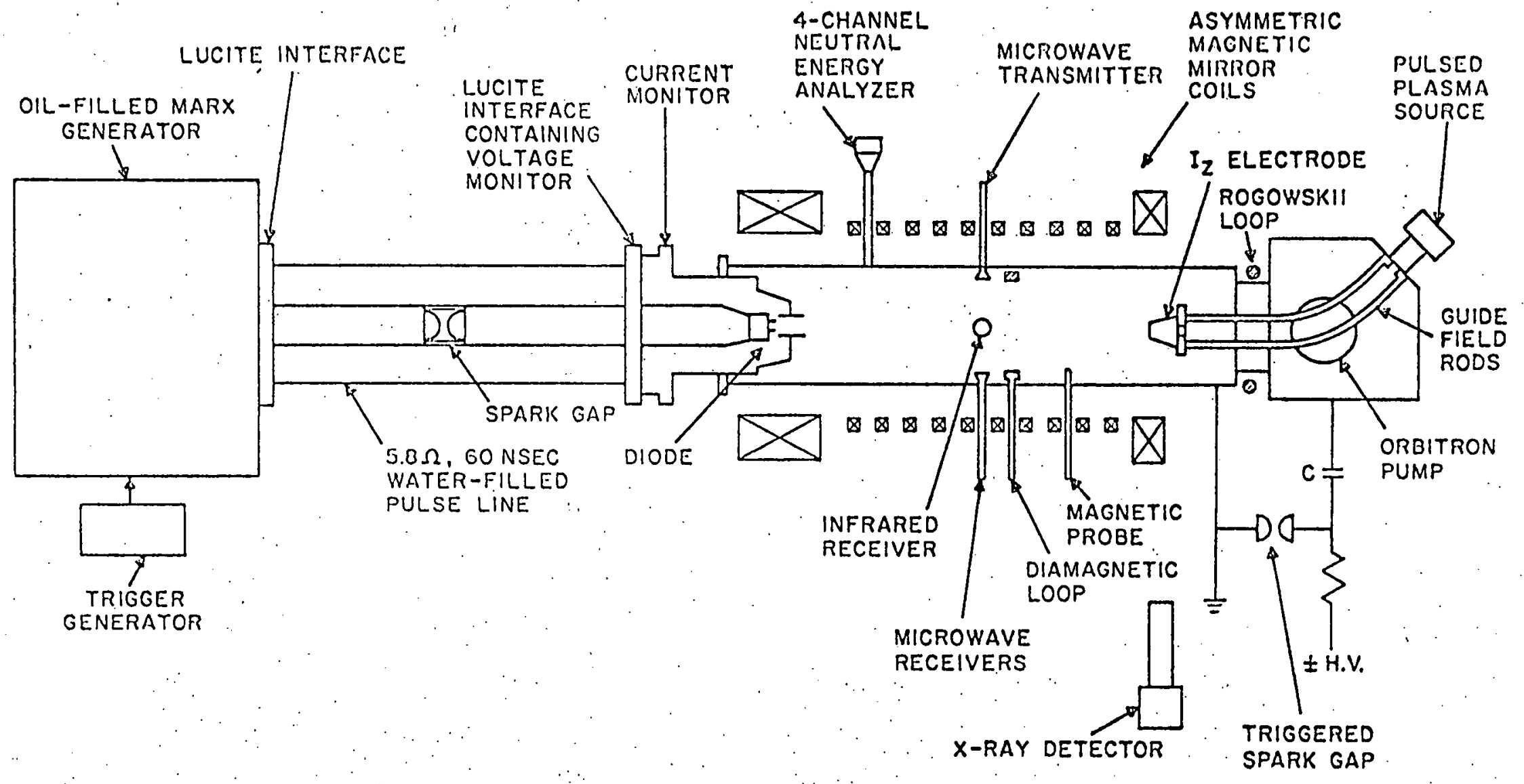

Figure 1. The Corneli turbulent heating-relativistic electron beam experiment. 
as the THM-III ${ }^{11}$ in that the capacitor bank acts as a current source and the anomalous resistivity appears as an increase in voltage between. the ends of the plaspa column. Figure $2 a-b$ displays oscilloscope traces of the electron beam diode voltage and current and the turbulent heating voltage and current. The period of anomalous resistivity as shown in Figure $2 b$ normally lasts a few hundred nanoseconds after which time the instability. is quenched and the voltage across the plasma colum rapidly falls to its pre-turbulence level. During nomal turbulent heating, measurement of the plasma diamagnetisn reveals perpendicular energies of the order $10 \mathrm{~J}$. and measurement of the ion energy distribution indicates the presence of hot ions $\left(T_{i} \sim 1-2 \mathrm{keV}\right)$.

For this experiment a $2.5 \mathrm{~cm}$ foil-less diode vith a carbon cathode was used to provide an electron beam matched to the size of the plasma column. The operation of the diode depends critically on the presence of plasna within the enode-cathode gap, which unfortunately precludes the use of calorimeters, Faraday cups or damage plates, which would block the flow of plasma. The operation of the accelerator and diode, and the resulting beam propagation and heating with this plasma configuration have been described else:vere. 1,2

It is possible to vary the time of beam injection with respect to the time of application of the turbulent heating voltage, designated $E_{Z}$. The delay time $\Delta t$ is defined as the time interval between the peak of the $E_{Z}$ voltage "spike" (i.e., peak plasina turbulence) and the time of beam injection. For nezative voltages applied to the $\mathrm{E}_{Z}$ electrode, the electrons in the turbulent heating current counterstrean with the beam 


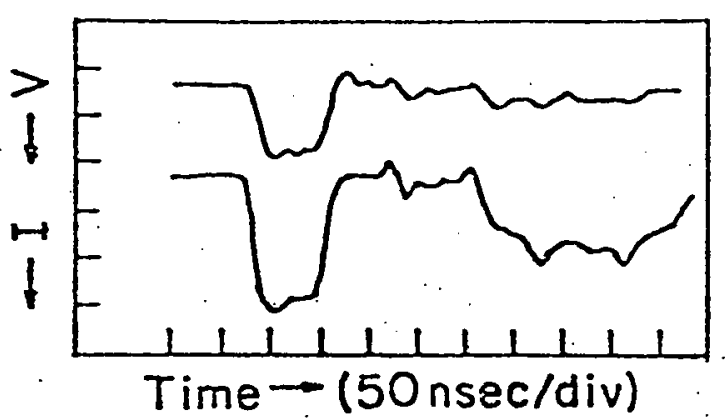

(a)

Vertical Scales
Voltage: $V \rightarrow(275 \mathrm{kV} /$ div $)$
Current: $I \rightarrow(20.8 \mathrm{kA} /$ div $)$

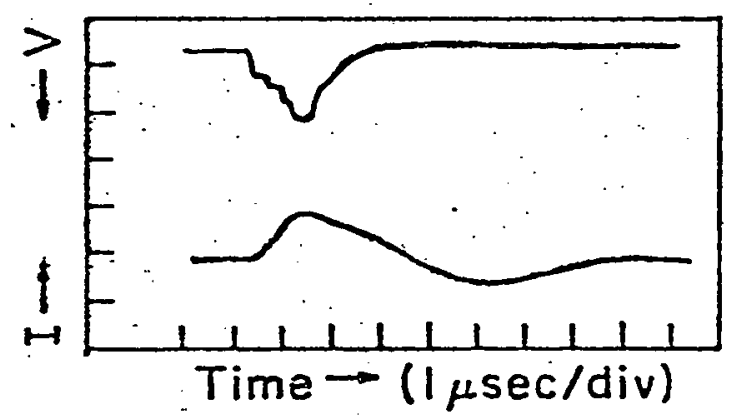

(b)

Vertical Scoles

Voltage: $V \rightarrow(20 \mathrm{kV} /$ div $)$

Current: $I \rightarrow(14 \mathrm{kA} /$ div $)$

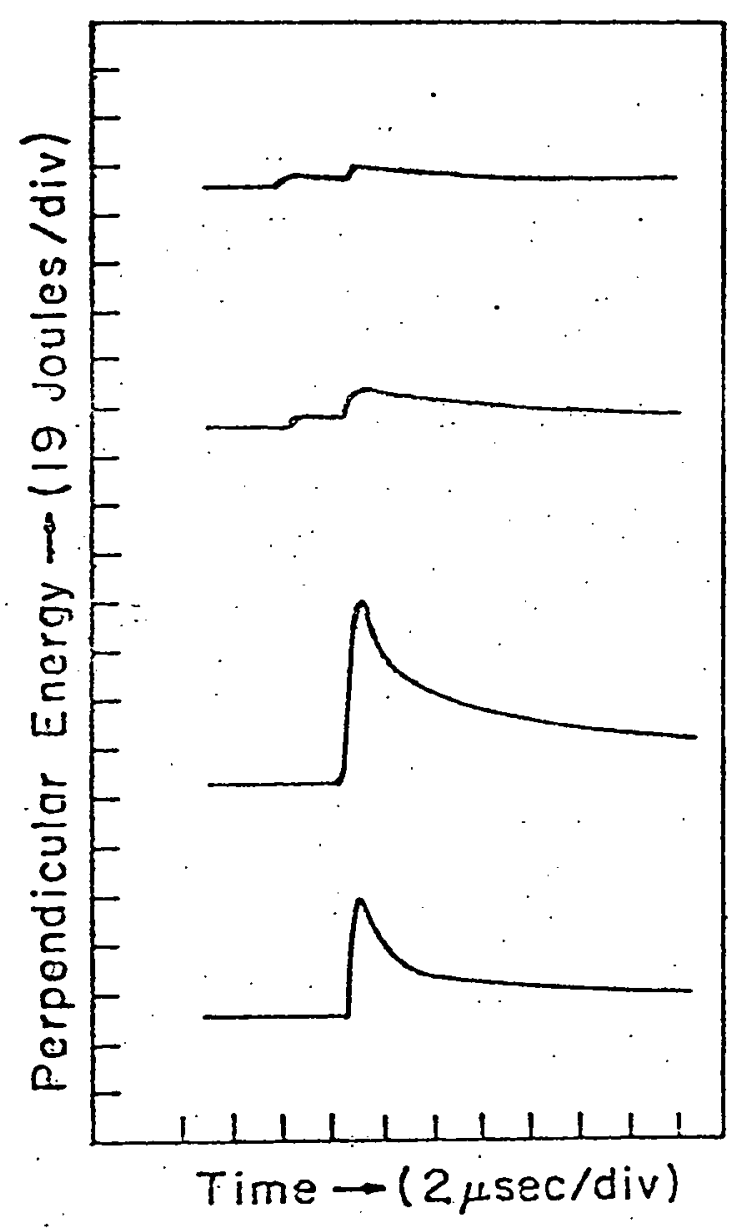

(c)

(d)

(e)

(f)

Eigure 2. Osc illoscope traces of a) electron beam diode voltage and current, b) Turoulent neating voltaze and current, c) Plasma dianagnetisn for it $\gg 0$, d) Plasma diamagnetism for $(t)>0$, e) Plasma dianagnetism for $\Delta=\sim 0$, f) Plassa diamagnctism for $\dot{\Delta t}<0$. 
primary electrons, but flow in the same direction as the beam return current during the pulse. Conversely, the opposite is true when the $\mathrm{E}_{2}$ electrode goes positive.

Figure 2c-f shows a set of oscilloscope traces of plasma diamagnetisn vs. time for different beam injection times. With $E_{Z}$ positive, plasma heating is relatively poor (less than that for beam heating alone) throughout the range of $\Delta t$ allowable with our equipment, and for beam injection before turbulence, heating gradually improves vith increasing negative $\Delta t$. With $E_{Z}$ negative, a sharp reduction in heating is observed when the beam is injected after pak turbulence (Figure 3). Figure 4 shows this effect for $\Delta t$ positive under a different set of. plasma conditions. Figure 5 shows data fron the sane run compared with a beam heating run using the same diode. It is apparent that, at a given level of diode current, injection of the bean into a highly turbulent plasma results in heating in excess of the sur of beam heating and turbulent heating alone. Figure 6 indicates the igproverent in ion heating observed when the bean is injected into a turbulent plesra, and Figure 7 shows comparative ion energy distributions for turbulent heating and lowlevel beam heating. The charge exchange reutral atom diagnostics employed are described elserhere. 11,12

Some features of this behavior are readily explainable, but others are much more speculative. The uniformly poor heating for bean injection long after peak turbulence is due to diode closure.: The diode impedance falls to low values ( $z_{\text {diode }}<z_{\text {pulse line }} \approx 5.8 \Omega$ ), suggesting that the turbulent heating current has filled the diode vith high-Z impurities. This is diode closure, and therefore the bean does not 


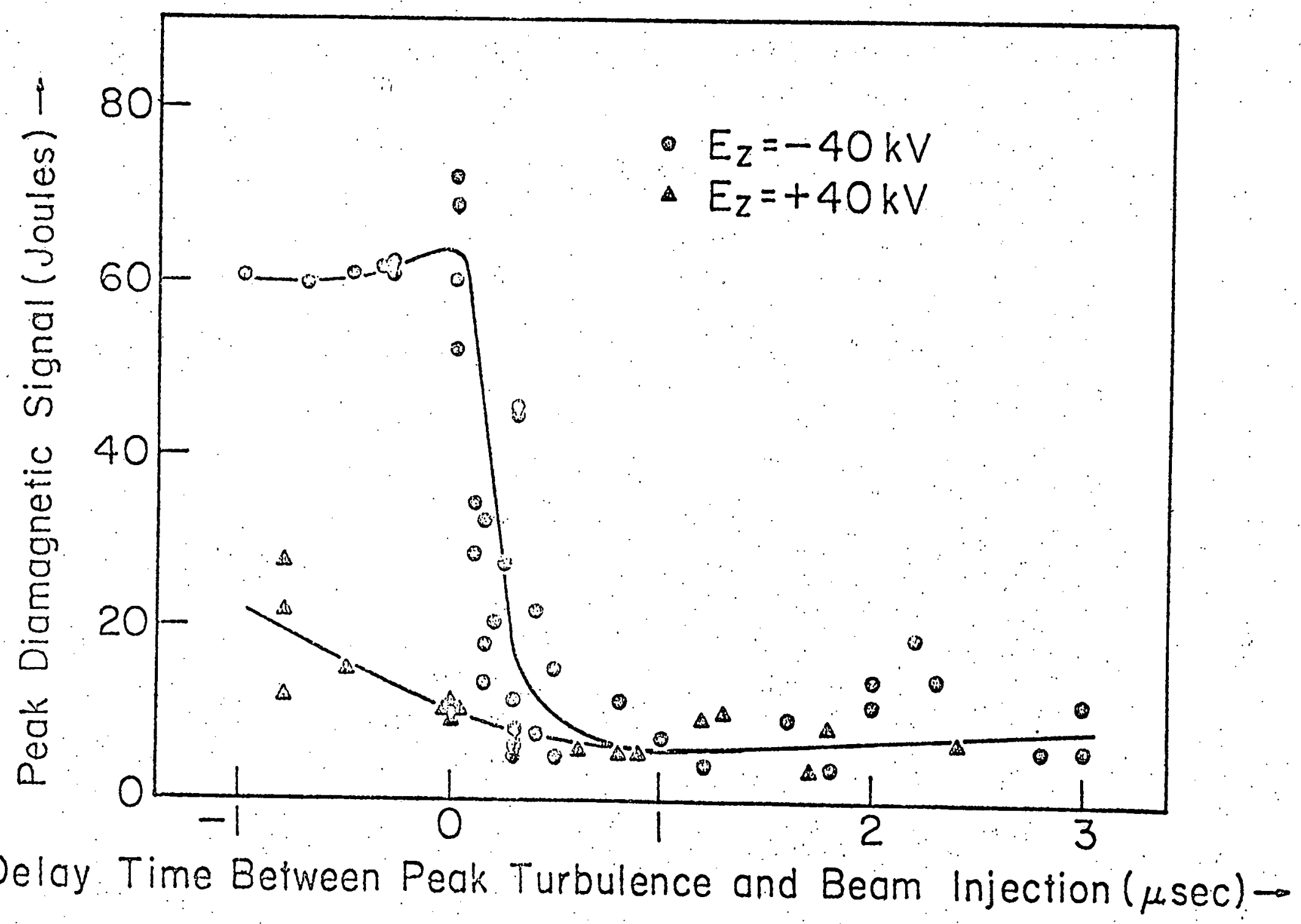

Figure 3. Peak plasma dlamagnetism vs, delay time between peak turbulence and electron beam injection for a $2.5 \mathrm{~cm}$ aperture foil-less diode. 


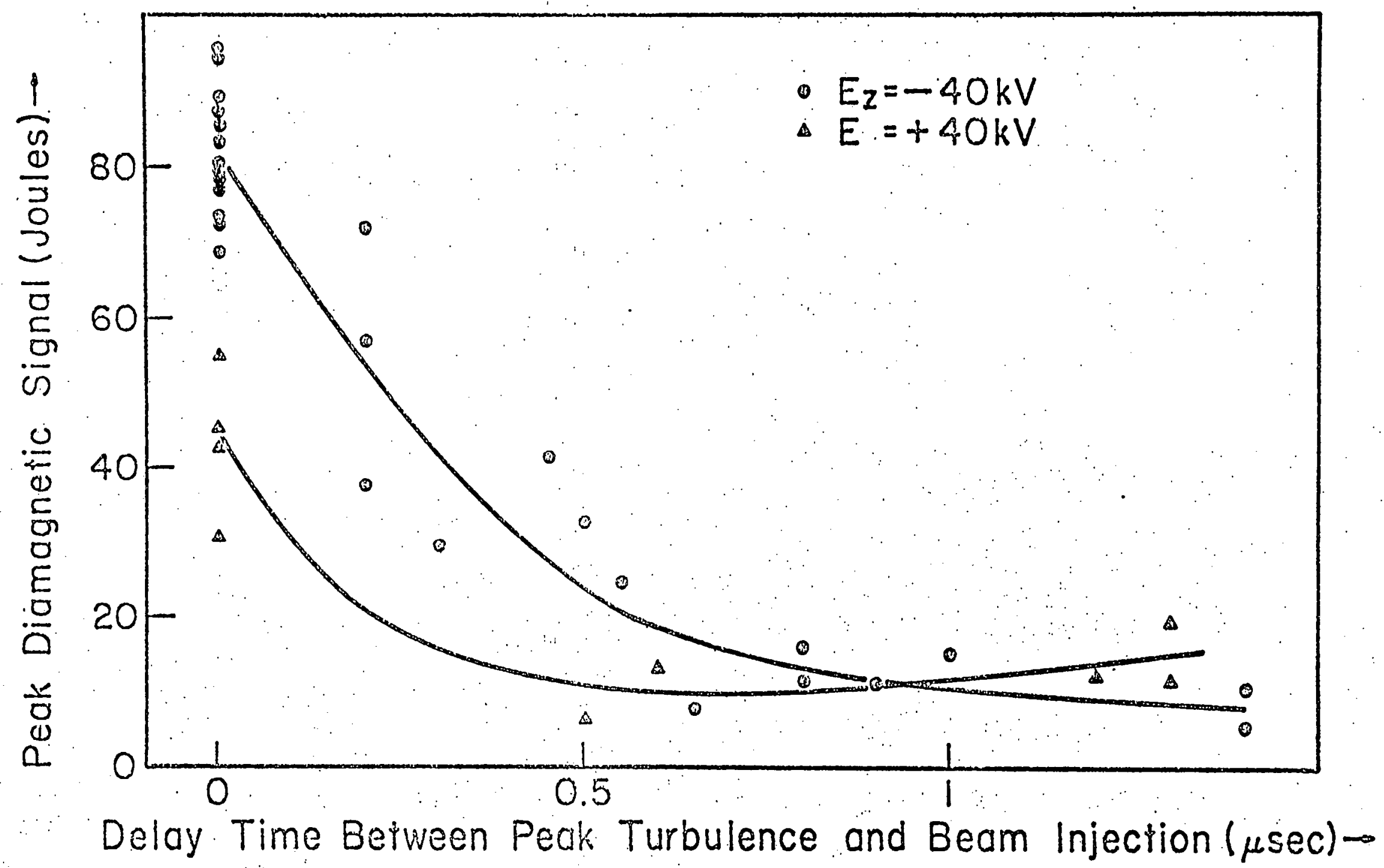

Figure 4. Peak plasma diamagnetisin vs. delay time between peak turbulence and electron beam infection for a $2.5 \mathrm{~cm}$ aperture foll-less dlode. 


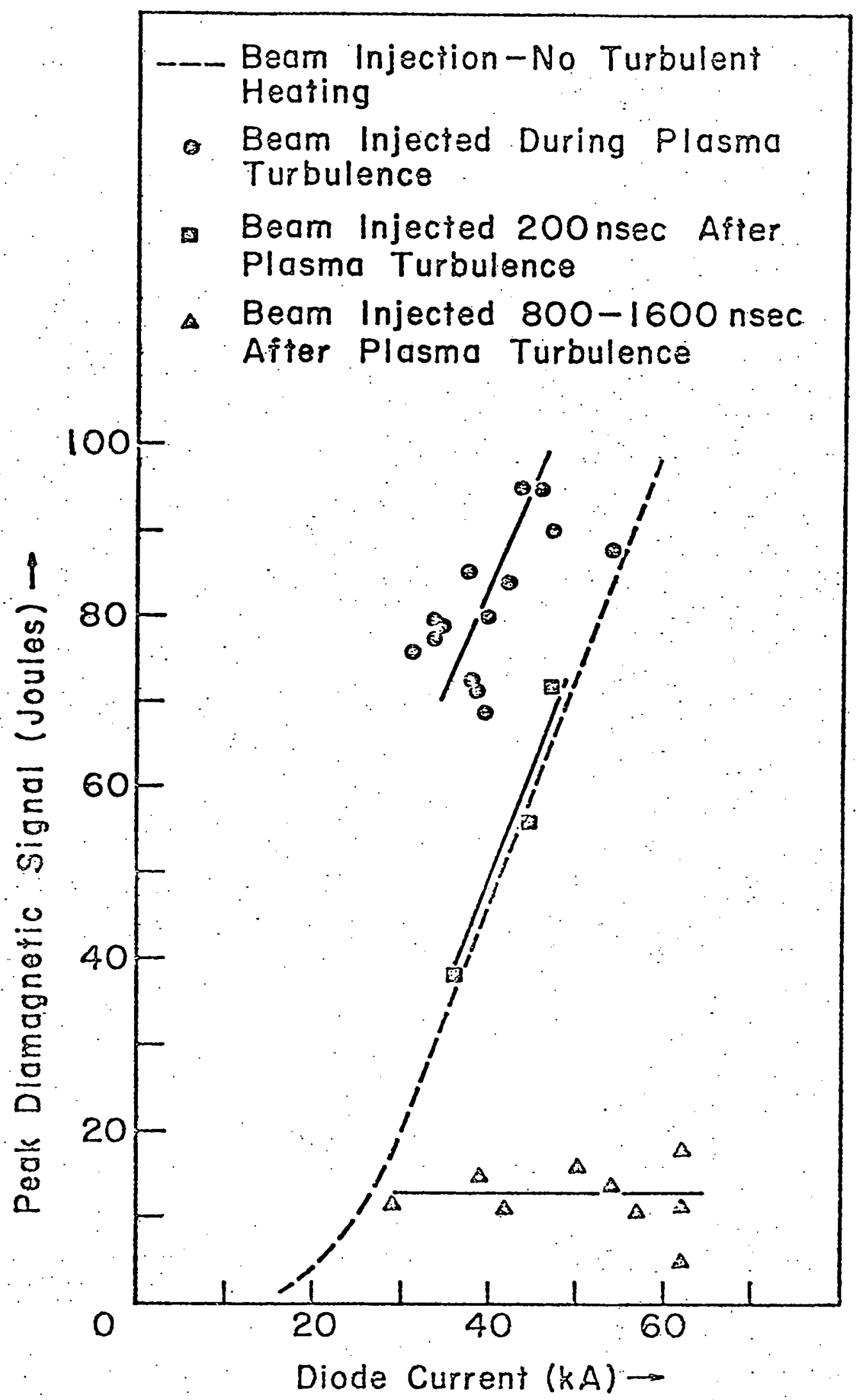

Figure 5. Peak plasma dianagnetisn vs. diode current for various beam injection times compared vith nornal beani heating using data from Figure 4. 


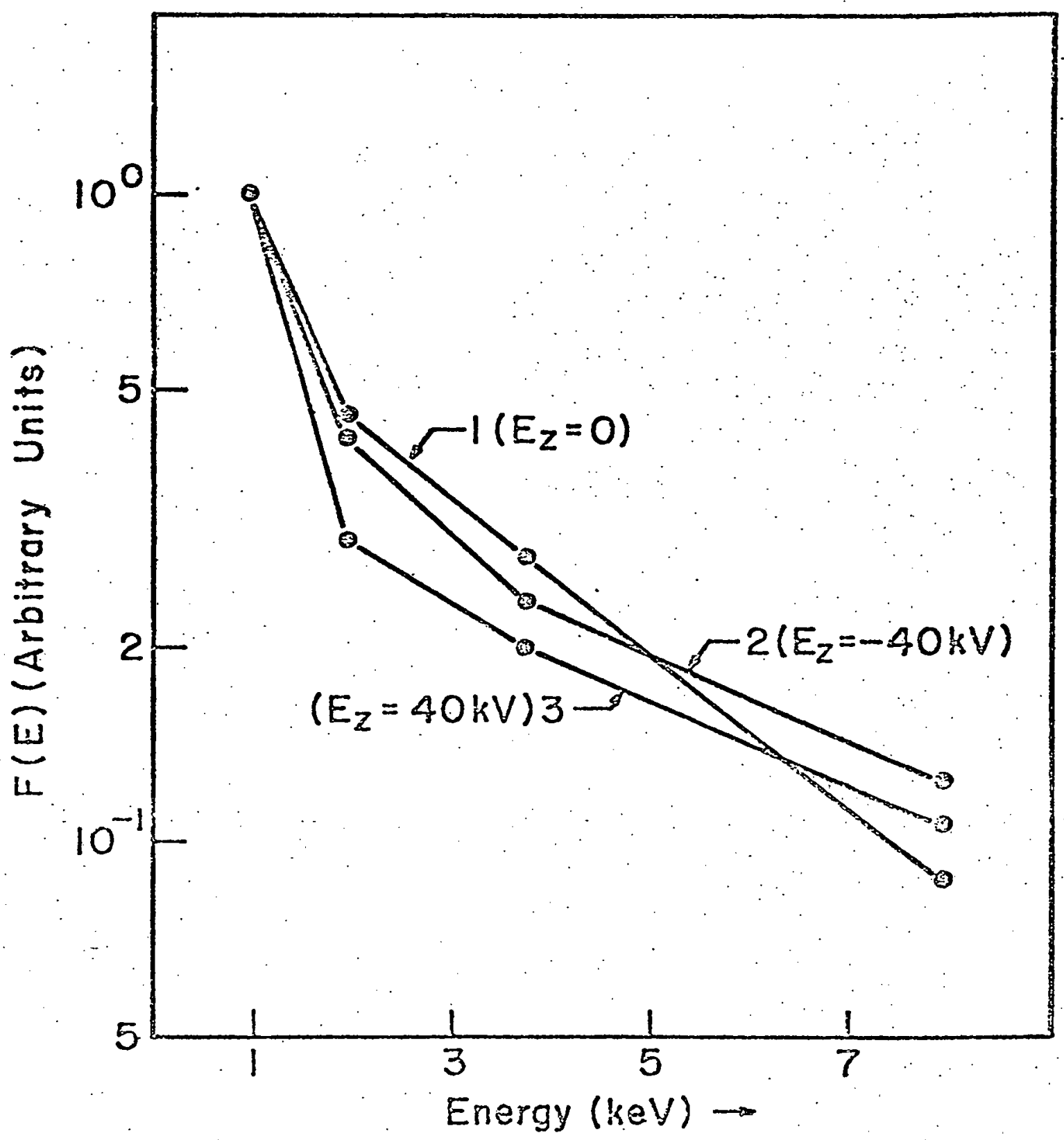

Figure 6. Ion distribution function vs. energy for beam injection with (1) no applied turbulent heating, (2) Z-discharge current opposing the relativistic bean current during peak turbulence, (3) Z-discharge current aligned with the relativistic beam current during peak turbulence. 


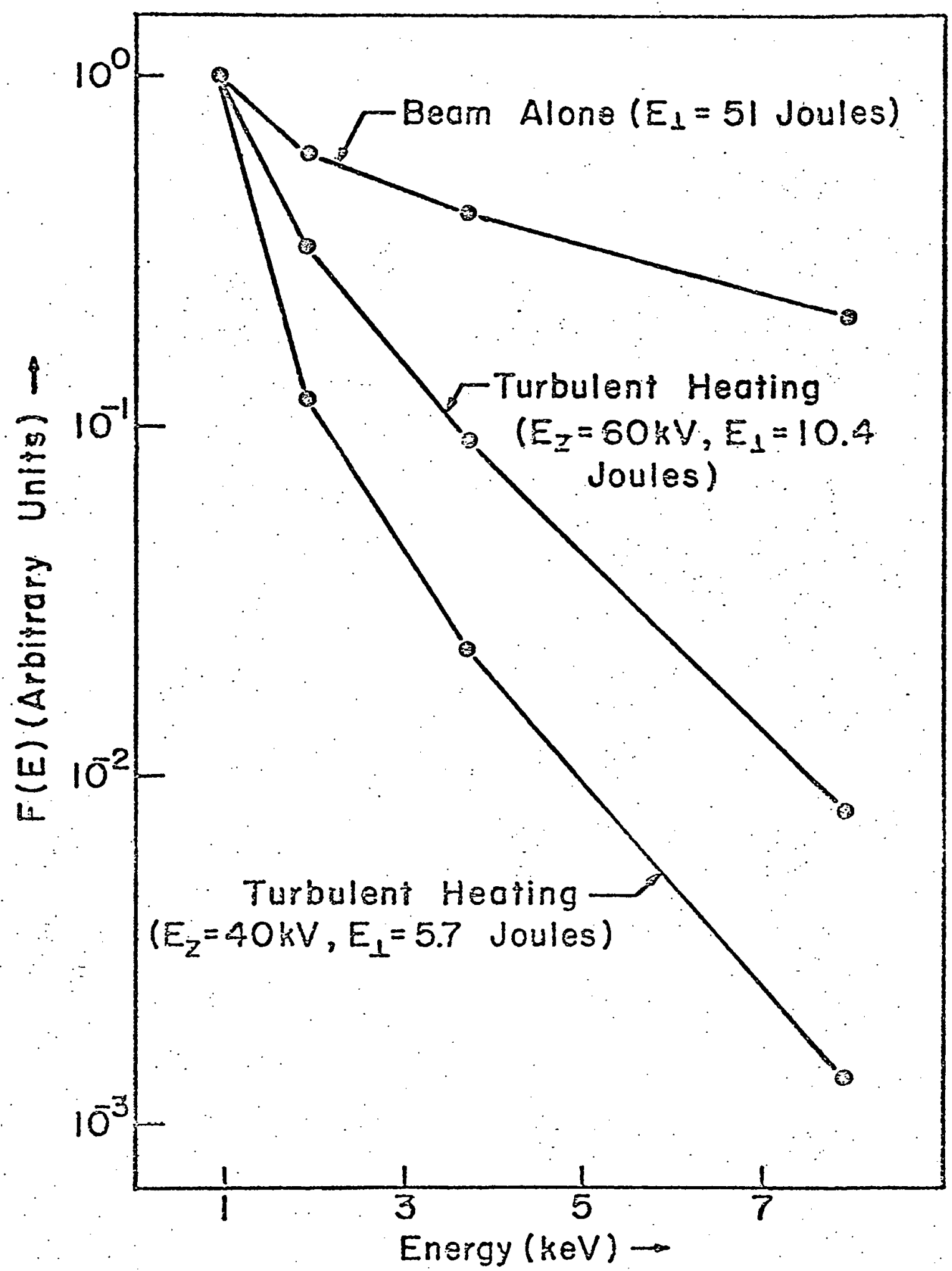

Figure 7. Ion distribution function vs. energy for bean plasma heating compared to plasma turbulent heating. 
propagate. Diamagnetic signals observed under sucin conditions show a rise in perpendicular energy due only to turbulent heating. That is, the signal shows no further increase following the time of beam injection.

The reasons for enhanced heating for beam injection during turbulence are more obscure. We offer the following speculations on the mechanisns at work:

1). Plasma heating, whether due to the beam return current or the turbulent heating current, is essentially an "ohnic" dissipative process. The power density delivered to the plasma is $\left(I_{\text {return }} \text { current }+I_{\text {turb. heat }}\right)^{2} n$, where $r_{\text {is }}$ is the plasma resistivity. $\eta$ increases rapidly during turbulence, then falls abruptly. It should be noted that $n$ is an increasing function of the net drift current. In addition, when the two currents are in the same direction, the total electron drift velocity increases, possibly pemitting higher temperatires to be attained before termination of the instability responsible for the turbulence. Wren the two currents oppose each other the opposite would be expected. As discussed above, the beam current falls rapidly for injection tines later than the turbulence period. We therefore might expect an enhancement in heating for bean injection at $\Delta t=0$, a rapid decline in heating vith positive $\Delta t$, and a nore moderate decline in heating for negative $\Delta t$, with an eventual retura to normal beam heating lavels for highly negative $\Delta t$, (in this experiment we were technically unable to inject the beam earlier than 1 microsecond before peak turbulence and still make useful measurenents).

2) From previous turbulent heating reasurements ${ }^{1.4}$ it is knom that 
ion-acoustic Eluctuations exist only during high turbulence periods. The possible result of the interaction of these vaves with the spectrum formed by the bean-plasma interaction may be enhanced heating levels.

In sumary, the interaction of a relativistic electron bean and. a turbulent plasma results in enhanced heating, depending on the direction of the turbulent heating current. This probably results, not only from the increased net heating current; but also from a change in the ratio of the drift velocity to the sound speed and the correspondtng increase in the plasma resistivity $n$. Additional evidence of this is the enhancerent of the ion energy distribution over that observed in nomal beam heating.

The authors wish to thank Prof. R. N. Sudan and Prof. N. Rostoker for valuable discussions, and Dr. S. H. Robertson and M. A. Greenspan for assistance witi the neutral particle energy analyzer.

This research was supported by the U. S. Atomic Energy Comission and the Office of Naval Research. 


\section{References}

1. P. Korn, F. Sandel and C. B. Wharton, to appear in Proceedings Sixth European Conference on Controlled Fusion and Plasma Physics, Moscow, USSR, July 30. - Aug. 3, 1973.

2. P. Korn, F. Sandel, and C. B. Wharton submitted for publication.

3. A. T. Altyntsev et al., Pis'ma 2h. Eksp. Teor. Fiz. 13, 197 (1971) JETP Lett. 13139 (1971), and in Proceedings of the Fourth International Conference on Plasma Physics and Controlled Nuclear Fusion Research, Madison, Wisconsin, 1971 (International Atomic Energy Agency, Vienna, 1972), Vol. 2, p. 309.

4. C. A. Kapetanakos, and D. A. Hamner to be published in Applied Dhysics Letters July 1, 1973, and C. A. Kapetarakos et a1., Bull. Amer. Phys. Soc. 17, 1032 (1972).

5. G. C. Goldenbaum et al., Bull. Amer. Phys. Soc. 17, 1031 (1972).

6. C. Stallings et al., Bull. Aner. Pnys. Soc. 17, 1031 (1972):

7. R. V. Lovelace and R. N. Sudan, Phys. Rev. Lett. 27, 1256 (1971).

8. A. A. Ivanov and I. I. Rudakov, Sov. Phys. JETP 31, 715 (1970). 
9. J. Guillory and G. Benford, Plasma Physics 14, 1131 (1972).

10.: L. E. Thode and R. N. Sudan, Phys. Rev. Lett. $\underline{30}, 732$ (1973).

11. S. Robertson, P. Korn and C. B. Wharton to appear in IEEE Transactions on Plasma Science.

12. C. Wharton et al., Proceedings of the Fourth International Conference on Plasma Physics and Controlled Nuclear Fusion Research, Madison, Wisconsin, 1971 (International Atomic Energy Agency, Vienna, 1972), Vol. 2 p. 25 .

13. Manufactured by Maxie11 Laboratories, Inc.

14. C. B. Tharton, D. Prono, and F. Sandel, in Proceedings of the Ninth Intermational Conference on Pnenomena in Ionized Gases, Jucharest (1969), p. 649 (unpublished). 
THE INTERACTION OF A RELATIVISTIC ELECTRON BEAM AND A FULLY IONIZED PLASMA*

**P. Korn, F. Sandel and C. B. Wharton Laboratory of Plasma Studies Cornell University. Ithaca, New York 14850

*Submitted to IEEE Trans. on Nuclear and Plasma Sciences. **Present address Maxwell Laboratories, Inc.

San Diego, California 92123

Research Supported by the U.S. Atomic Energy Commission DCTR, and the Office of Naval Research, Power Branc. 


\begin{abstract}
A strong interaction between an intense $(0.4 \leq \nu / \gamma \leq 2.2)$ relativistic electron beam and a fully ionized, mirror confined hydrogen plasma has been observed. The plasma was heated to multikilovolt average energies of (5-15 keV) per electron-ion pair, with optimum heating at the lowest effective diode impedance levels (high $\nu / \gamma$ ). The existence of hot ions was demonstrated, and microwave, far-infrared, and x-ray emissions were observed. Electron beam plasma heating was compared with direct axial current driven turbulent heating, and the interaction of the electron beam with a turbulent plasma was investigated.
\end{abstract}


CONTENTS

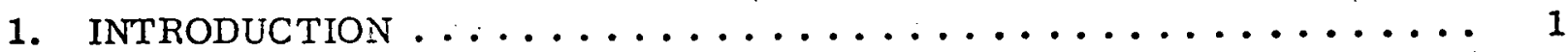

2. DESCRIPTION OF THE EXPERIMENT .................. 1

3. DIODE CHARACTERISTICS ..................... 5

4. PLASMA HEATING ......................... 5

5. RELATIVISTIC BEAM INTERACTION WITH A TURBULENT PLASMA . . 13

6. EFFECT OF VARIATION OF THE MAGNETIC MIRROR FIELD $\ldots \ldots . . .17$

7. TIME SCALE OF BEAM HEATING ................... 17

8. OBSERVATION OF MICROWAVE, FAR-INFRARED, AND HARD

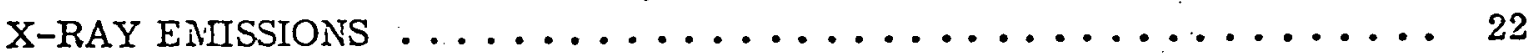

9. LOCALIZATION OF THE HEATING PROCESS . . . . . . . . . 26

10. CONTAINNIE ITT WITH AND WITHOUT THE MLAGNETIC MIRROR $\ldots \ldots$

11. CONCLUSIONS AND HEATING EFFICIENCY . . . . . . . . . . . . 28

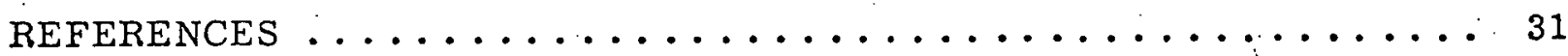




\section{ILLUSTRATIONS}

1. The Electron Beam and Turbulent Heating Machines $\ldots \ldots \ldots \ldots \ldots \ldots$

2. Summary of Diode Structures $\ldots \ldots \ldots \ldots \ldots \ldots \ldots \ldots \ldots \ldots \ldots$

3. Magnetic Mirror Field Profiles, (a) Unperturbed Field, (b) With Diode Structure, (c) One Mirror Removed . . . . . . . . . . . . . 4

4. Peak Diamagnetic Signal Versus Diode Current (Foil Diodes). Each Point Corresponds to One Machine Firing .................. 6

5. Peak Diamagnetic Signal Versus Diode Current ( $5 \mathrm{~cm}$ Foil-Less Diode). Each Point Corresponds to One Machine Firing . . . . . . . . . . . . 8

6. Peak Diamagnetic Signal Versus Effective Diode Impedance (Various Diodes). Each Point Corresponds to One Machine Firing ......... 9

7. Neutral Signals Observed $30 \mathrm{~cm}$ From the Diode . . . . . . . . . . . 10

8. Ion Distribution Function Versus Energy for Various Beam Heating Levels. Curves 1-5, $\mathrm{E}_{\perp}=82,74,40,30,13 \mathrm{~J}$, respectively, $1-4$ With $2.5 \mathrm{~cm}$ Foilless Diode and 5 Using a $5 \mathrm{~cm}$ Foiled Diode ............. 11

9. (a) Diode Voltage and Current Waveforms, (b) Plasma Diamagnetism, $\mathrm{E}_{\perp},<35 \mathrm{~J}$, (c), (d) Plasma Diamagnetism $\mathrm{E}_{\perp} \sim 35-60 \mathrm{~J}$, and (e) Plasma

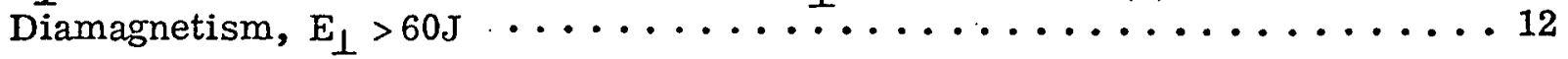

10. Comparative Neutral Energy Spectra for Beam Heating and Turbulent Heating - 14

11. Oscilloscope Traces for the Beam - Turbulent Heating Interaction

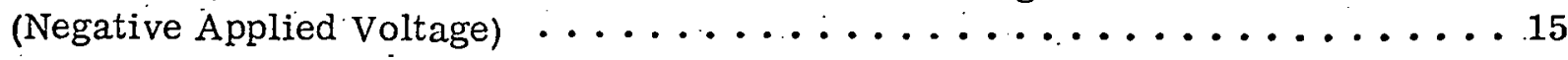

12. Effect of Beam Injection on the Highly Turbulent Plasma $\ldots \ldots \ldots \ldots \ldots$

13. Beam Injection Re-initiating Plasma Turbulence $\ldots \ldots \ldots \ldots \ldots \ldots$

14. Diode Current Versus Magnetic Mirror Field . . . . . . . . . . . . . 19

15. Peak Diamagnetic Signal Versus Magnetic Mirror Field $\ldots \ldots \ldots \ldots \ldots 20$

16. Peak Diamagnetic Signal Versus Diode Current (Showing the Effect of a Shortened Current Pulse) . .................... 21

17. Peak Microwave Detector Signal versus Diode Current $\ldots \ldots \ldots \ldots \ldots \ldots 23$

18. Peak Microwave Detector Signal Versus Mirror Field 5 . . . . . . 24

19. Time Synchronized Microwave and Far-Infrared Emission Oscillographs $\ldots 25$

20. Hard X-Ray Emission Corresponding to Discontinuities in the Diamagnetic Signal 27

21. Plasma Diamagnetic Signal With and Without the Magnetic Mirror . . . . . 29 


\section{INTRODUCTION}

Several recent experiments ${ }^{(1-5)}$ have demonstrated that is is possible to transfer an appreciable fraction of the energy contained in a relativistic electron beam into thermal energy of a target plasma. In this experiment, the interaction of an intense $(4 \leq \nu / \gamma \leq 2.2)$ electron beam with a dense $\left(\mathrm{n} \sim 5-8 \times 10^{13} \mathrm{~cm}^{-3}\right)$ plasma column has been studied. The plasma was contained in a magnetic mirror far from the wall of the machine in a hard vacuum background. These conditions are desirable for unambiguous interpretation of the diagnostics.

\section{DESCRIPTION OF THE EXPERIMENT}

The experiments were performed in the Cornell THM (Turbulent Heating Machine) ${ }^{(6)}$ which was operated single-ended. The hydrogen plasma was formed by operation of a pulsed valve and conical pinch plasma gun, (see Figure 1). A curved hexapole guide field separates neutral particles which are removed by a titanium gettering pump, allowing background pressures of $2-4 \times 10^{-7} \mathrm{~T}$. The plasma column is $\sim 4 \mathrm{~cm}$ in diameter and $150 \mathrm{~cm}$ long, with a sharply peaked radial profile. Normal turbulent heating is achieved by applying \pm 20 - $100 \mathrm{kV}$ from a $0.09-0.36 \mu \mathrm{F}$ capacitor bank to the conical electrode plasma limiter while the diode anode structure is at ground potential.

A Maxwell Laboratories 5.8 $\Omega$ POCOBEAM system consisting of a 16 stage oil insulated Marx generator and a $60 \mathrm{nsec} 5.8 \Omega$ water-insulated pulse line was used to generate the electron beam. A summary of the various field emission diodes used is shown in Figure 2. The ranges of diode voltage and current were 200 $500 \mathrm{kV}$ and $5-65 \mathrm{kA}$, respectively.

Three magnetic mirror field profiles are displayed in Figure 3, showing the unperturbed field, the effect of the diode support structure on the magnetic field, and the result of removing one of the mirrors, respectively. During normal operation, the minimum field in the mirror well was $2.7 \mathrm{kG}$. 


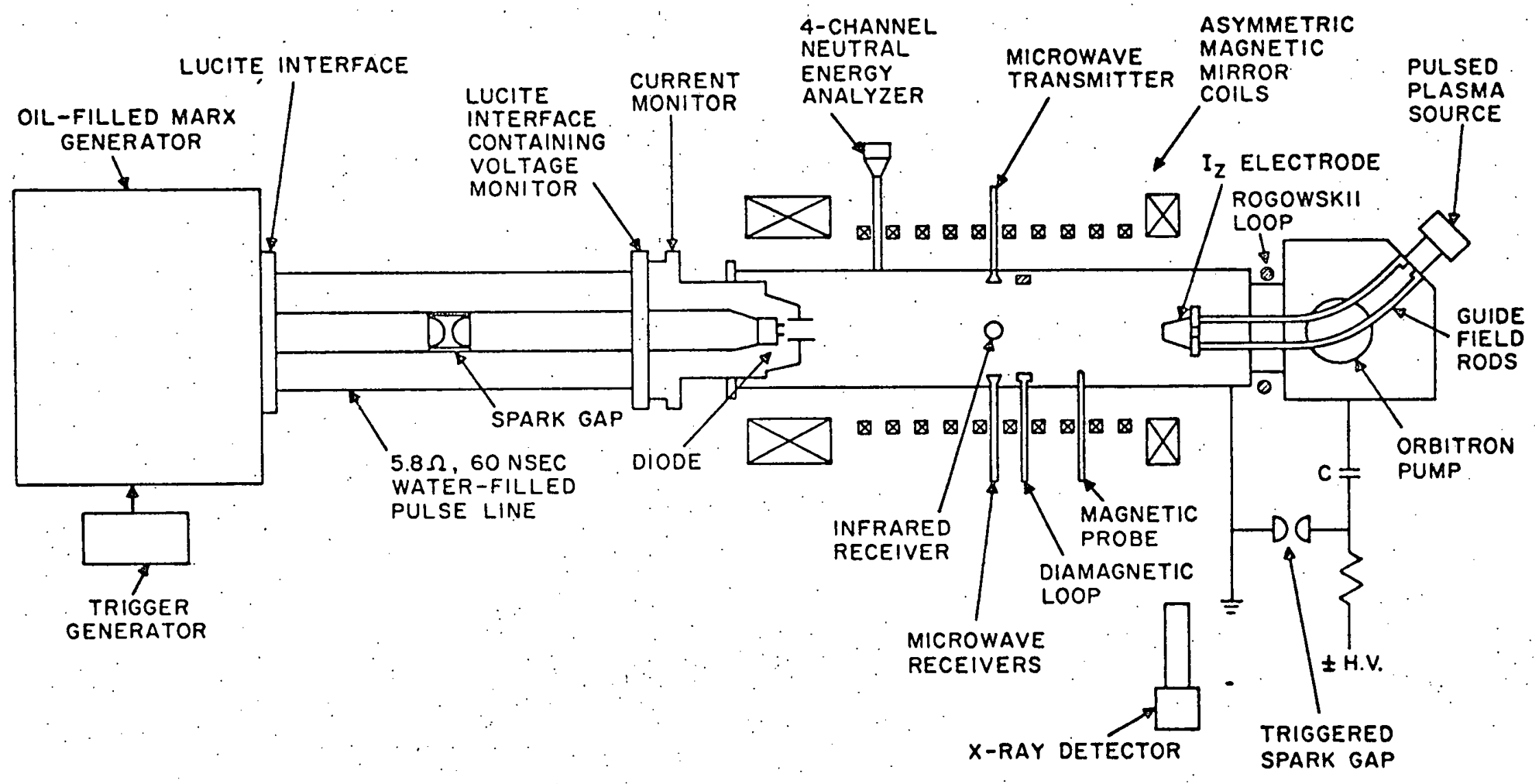

Figure 1. The Electron Beam and Turbulent Heating Machines. 

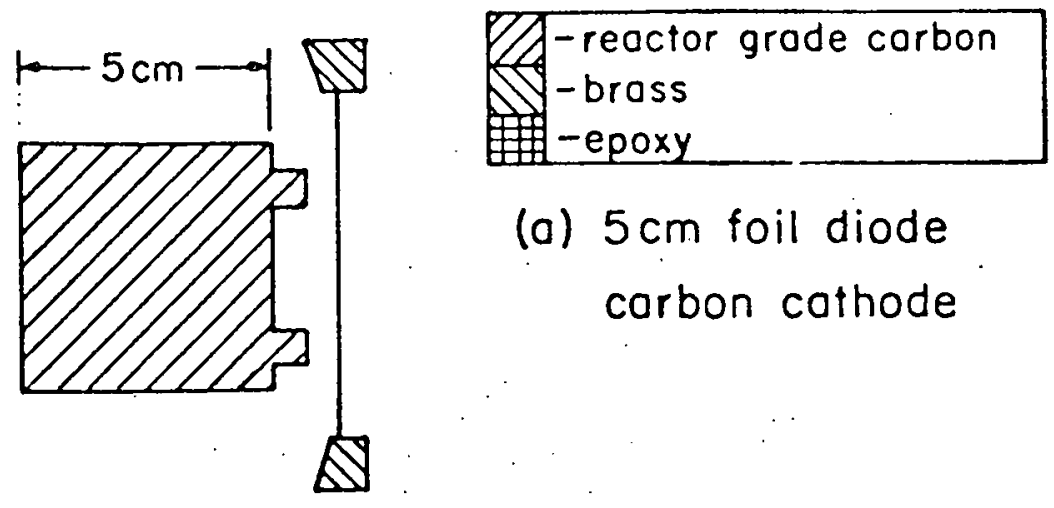

(a) $5 \mathrm{~cm}$ foil diode carbon cothode

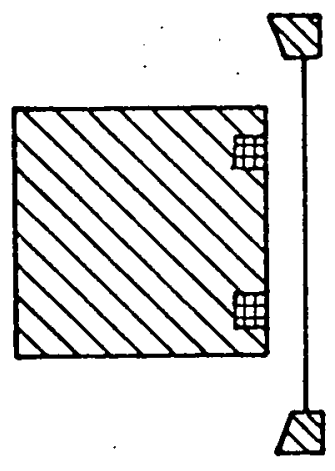

(b) $5 \mathrm{~cm}$ foil diode plasmo cathode

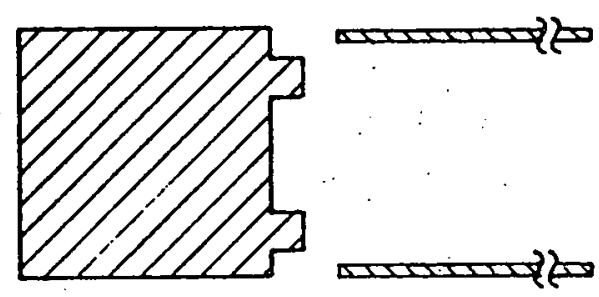

(c) $5 \mathrm{~cm}$ foil-less diode corbon cothode

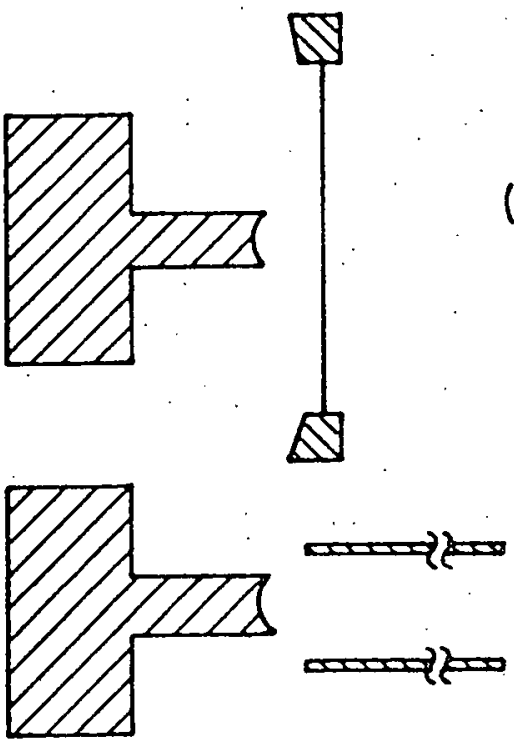

(d) $2.5 \mathrm{~cm}$ foil diode carbon cathode

(e) $2.5 \mathrm{~cm}$ foil-less diode carbon cothode

Figure 2. Summary of Diode Structures. 


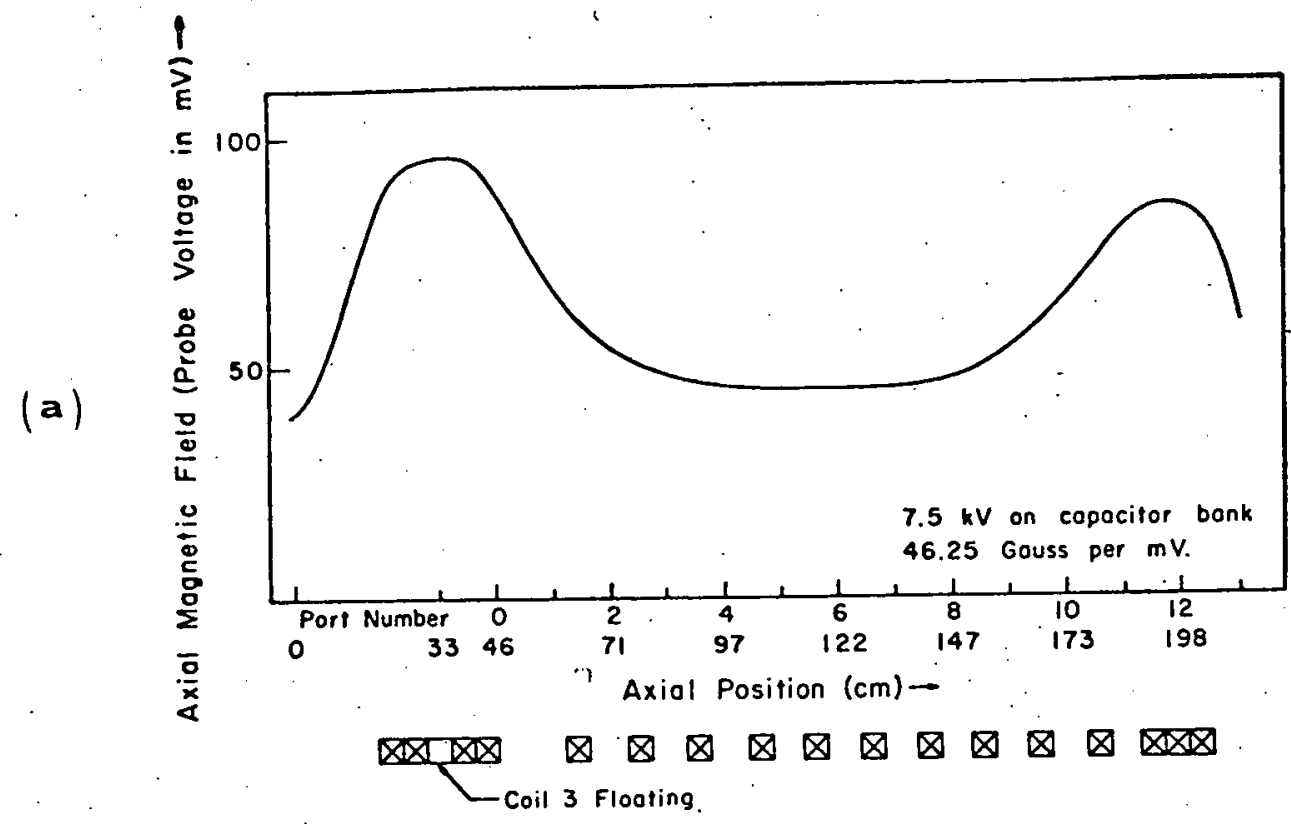

(b)

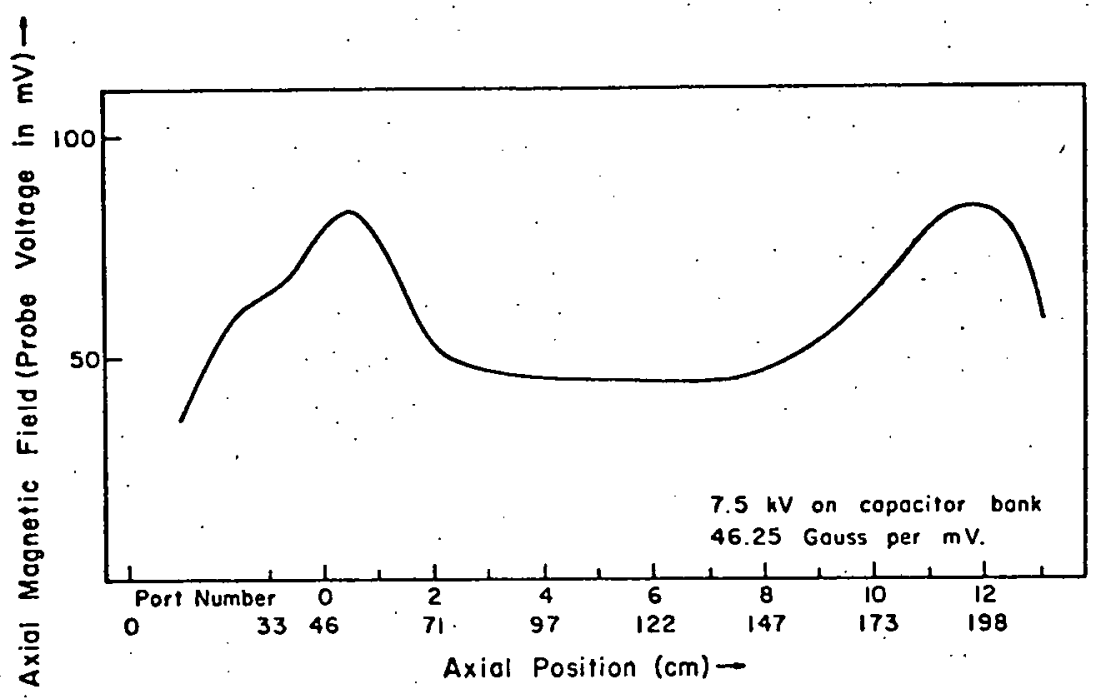

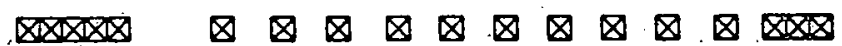

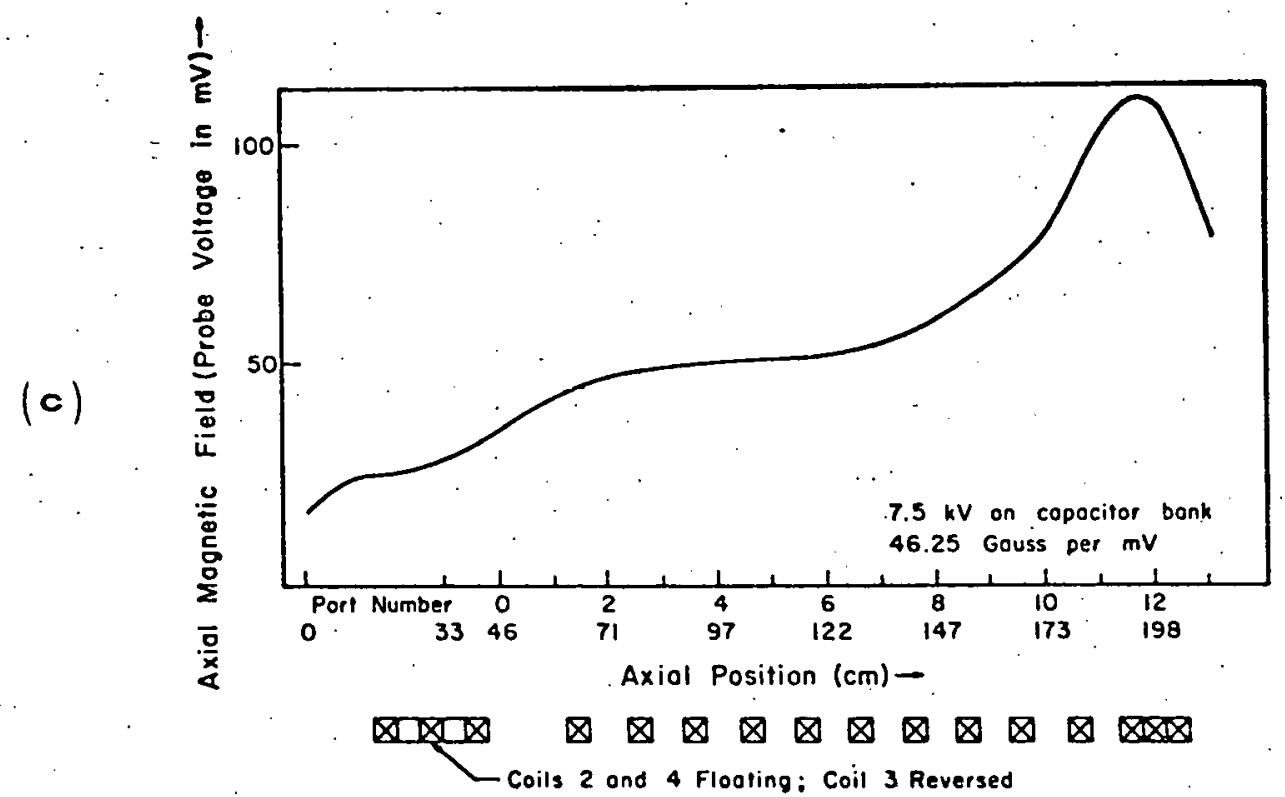

Figure 3. Magnetic Mirror Field Profiles, (a) Unperturbed Field, (b) With Diode Structure, . (c) One Mirror Removed. 
The ion energy distribution was measured by analyzing the fast neutral atoms arising from charge-exchange of the plasma ions on a background of krypton gas $\left(\mathrm{P} \sim 2 \times 10^{-5} \mathrm{~T}\right)$ introduced with a pulsed valve. Plasma diamagnetism, from which the total perpendicular plasma pressure was calculated, was measured with an electrostatically shielded compensated loop located near the machine midplane.

\section{DIODE CHARACTERISTICS}

Plasma heating was relatively insensitive to the diameter of the diode used. A more important parameter was the anode-cathode gap spacing, which was 0.5 $0.75 \mathrm{~cm}$ in these experiments. Foil diodes, if properly conditioned by increasing the diode current slowly over several shots, lasted an average of 10 shots for the $2.5 \mathrm{~cm}$ diode and 40 shots for the $5 \mathrm{~cm}$ diode before foil rupture. Currents up to $30 \mathrm{kA}$ were possible without foil rupture. The plasma cathode (epoxy-filled) rapidly deteriorated and as a result the carbon cathodes were favored.

Foil-less diodes were prepared by replacing the anode foil with a $10 \mathrm{~cm}$ long section of brass tubing. No effort was made to impede the flow of gun plasma into the anode-cathode gap. In fact, it was found necessary to have some plasma in the diode for proper beam propagation, and the diode impedance depended very critically upon its presence. The amount of plasma in the anode region could be controlled by varying the delay time between the firing of the plasma gun and the Marx bank trigger. Excessive plasma injection resulted in diode closure and produced high $(80-120 \mathrm{kA})$ diode currents and low $(200-300 \mathrm{kV})$ diode voltages. In such cases the beam did not propagate. Conversely, insufficient plasma resulted in a high impedance diode, also with little beam propagation.

\section{PLASMA HEATING}

Plasma heating in this configuration has been discussed in detail elsewhere. Additional data will be presented here. Figure 4 shows the plasma perpendicular energy as a function of diode current obtained using foil diodes. It is apparent that there is a threshold for heating at a diode current of approximately $8 \mathrm{kA}$. This is 


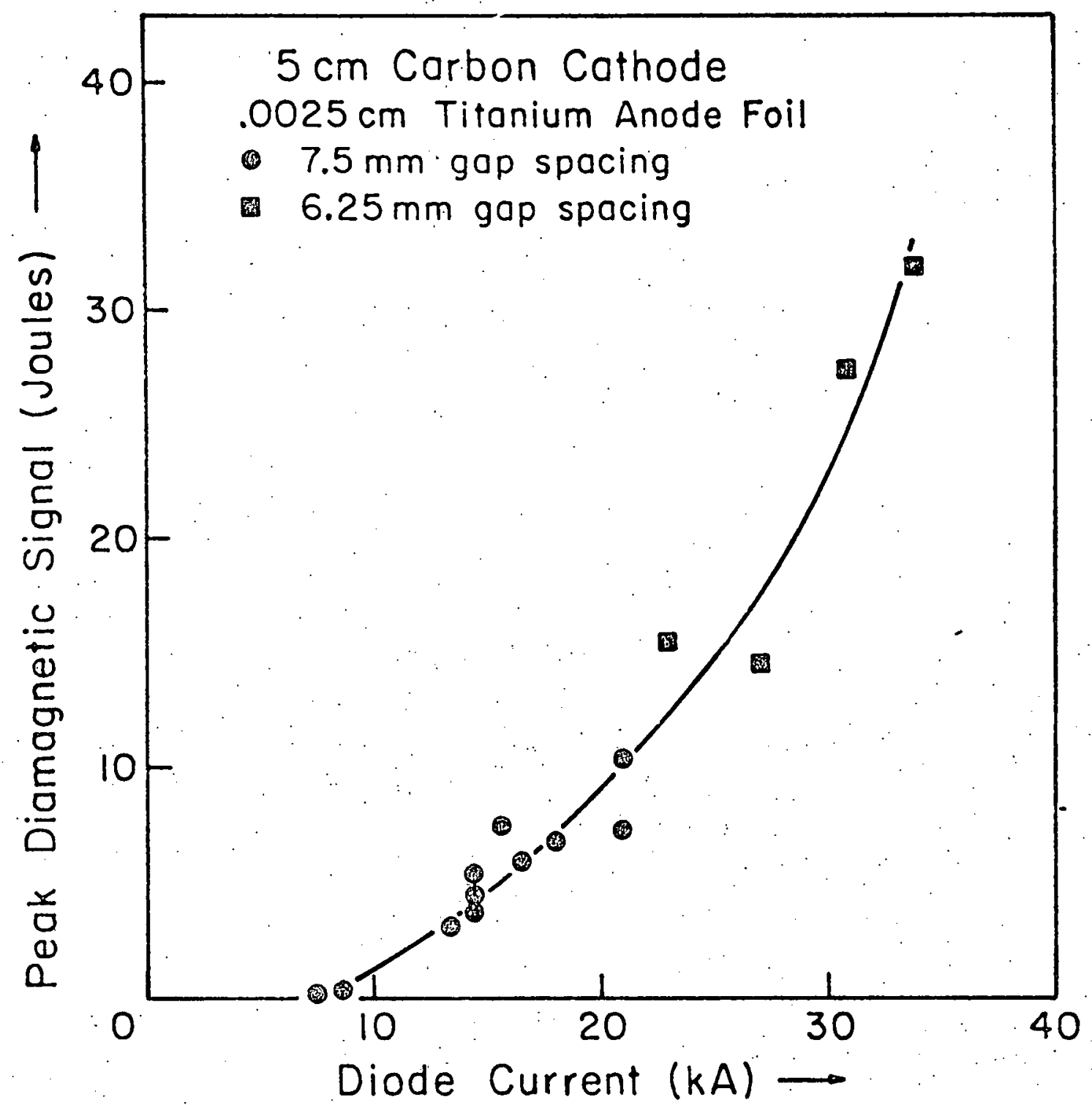

Figure 4. Peak Diamagnetic Signal Versus Diode Current (Foil Diodes). Each Point Corresponds to One Machine Firing. 
comparable to the threshold observed for turbulent heating in this machine. (8) Foilless diodes were used to repetitively operate at higher currents. A plot of perpendicular energy versus diode current obtained using the $5 \mathrm{~cm}$ diode is shown in Figure 5. The perpendicular energy is seen to monotonically increase with increased diode current without apparent limit. Increased diode current could be obtained by increasing the charging voltage of the Marx bank or by injecting more plasma into the diode, thus lowering the diode impedance. The eventual plasma diamagnetism and energy transfer efficiency depends on the effective diode impedance, and thus on the power in the beam, with the lowest impedance levels obtainable without diode closure providing the best heating. This effect is relatively insensitive to the particular diode configuration used, as shown in the composite curve in Figure 6. It should be noted, however, that the diode impedance and the effect of plasma in the diode on beam propagation are of necessity interrelated in determining the eventual plasma heating in these foil-less diode experiments.

Four points on ion energy distribution curve could be obtained on each shot using a 4-channel charge-exchange neutral energy analyzer. (9) Typical neutral signals are shown in Figure 7. The first peak in each trace corresponds to the flux of hydrogen neutrals with later peaks corresponding to the arrival of various impurities. Several such distributions are displayed in Figure 8. Curves 1-4 $(82 \mathrm{~J}, 75 \mathrm{~J}, 40 \mathrm{~J}$, and $30 \mathrm{~J}$ respectively) are from the run of Figure 5 , while curve 5 (13J) is a foil-diode shot included for comparison. As apparent saturation in ion heating in this energy interval is evident for perpendicular energies $>40 \mathrm{~J}$. At this level the observed diamagnetic signal undergoes a change in time-dependence as shown in Figure 9, indicating reduced confinement time for several microseconds following heating. However, the peak diamagnetic signal shows no saturation. There appear to be four possible explanations for this behavior. First, at high heating levels the ion loss rate may increase, possibly due to an instability. Second, the large diamagnetic signal may be due to heated plasma electrons, which have a rapid loss rate following heating. Alternatively, portions of the ion distribution outside the energy range shown in Figure 8 may be preferentially populated. 


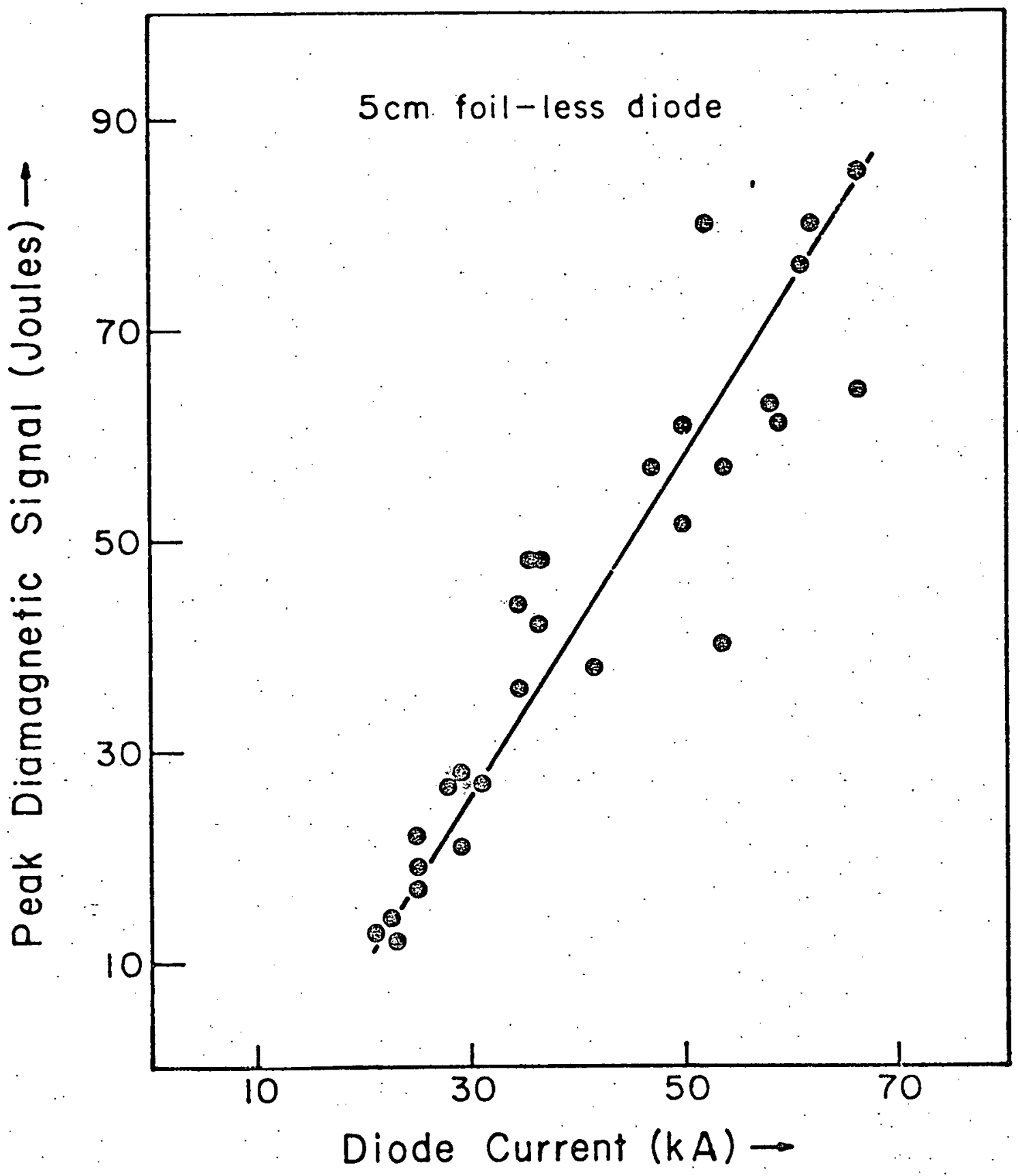

Figure 5. Peak Diamagnetic Signal Versus Diode Current (5. $\mathrm{cm}$ Foil-Less Diode). Each Point Corresponds to One Machine Firing. 


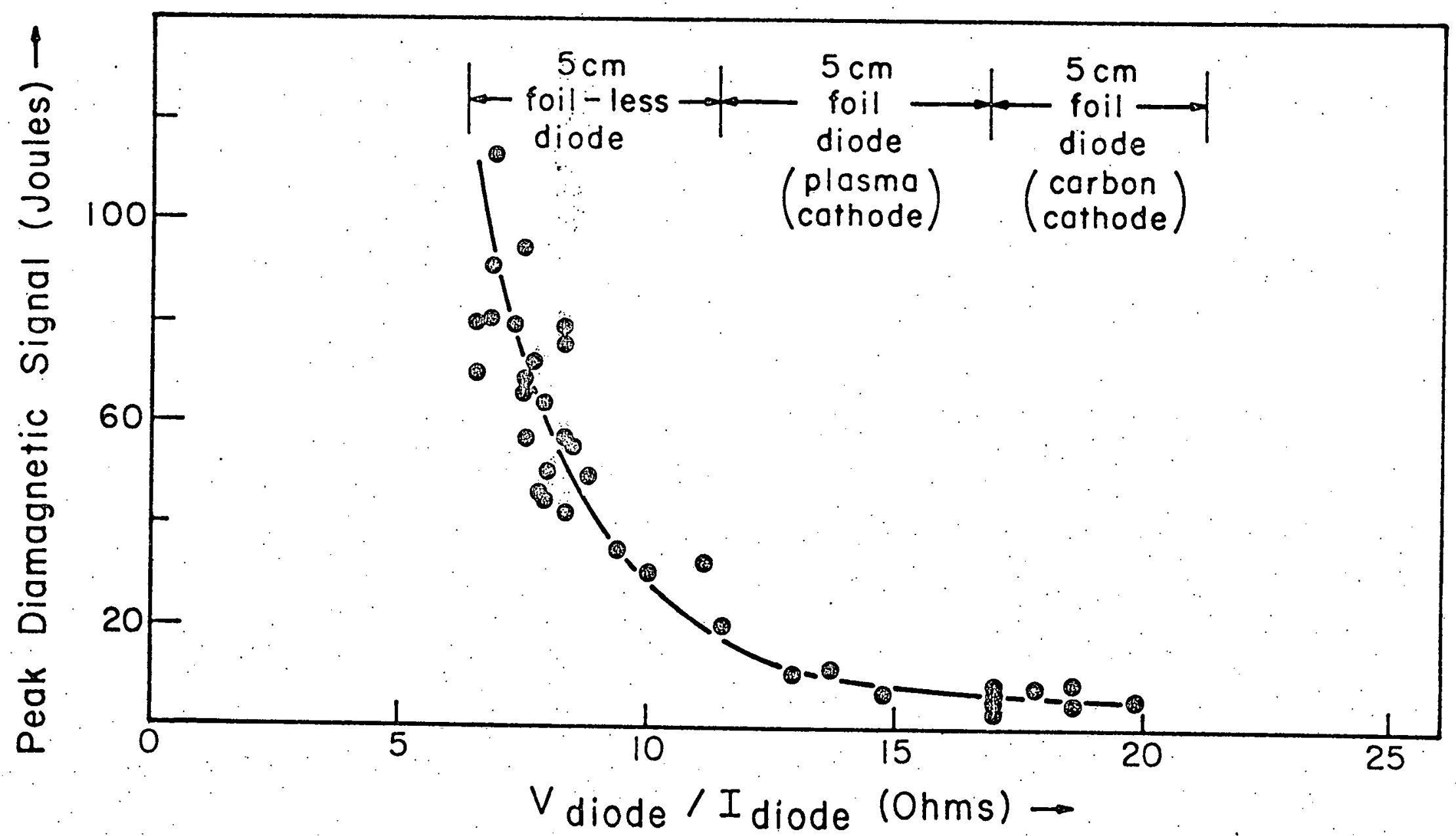

Figure 6. Peak Diamagnetic Signal Versus Effective Diode Impedance (Various Diodes). Each Point Corresponds to One Machine Firing. 


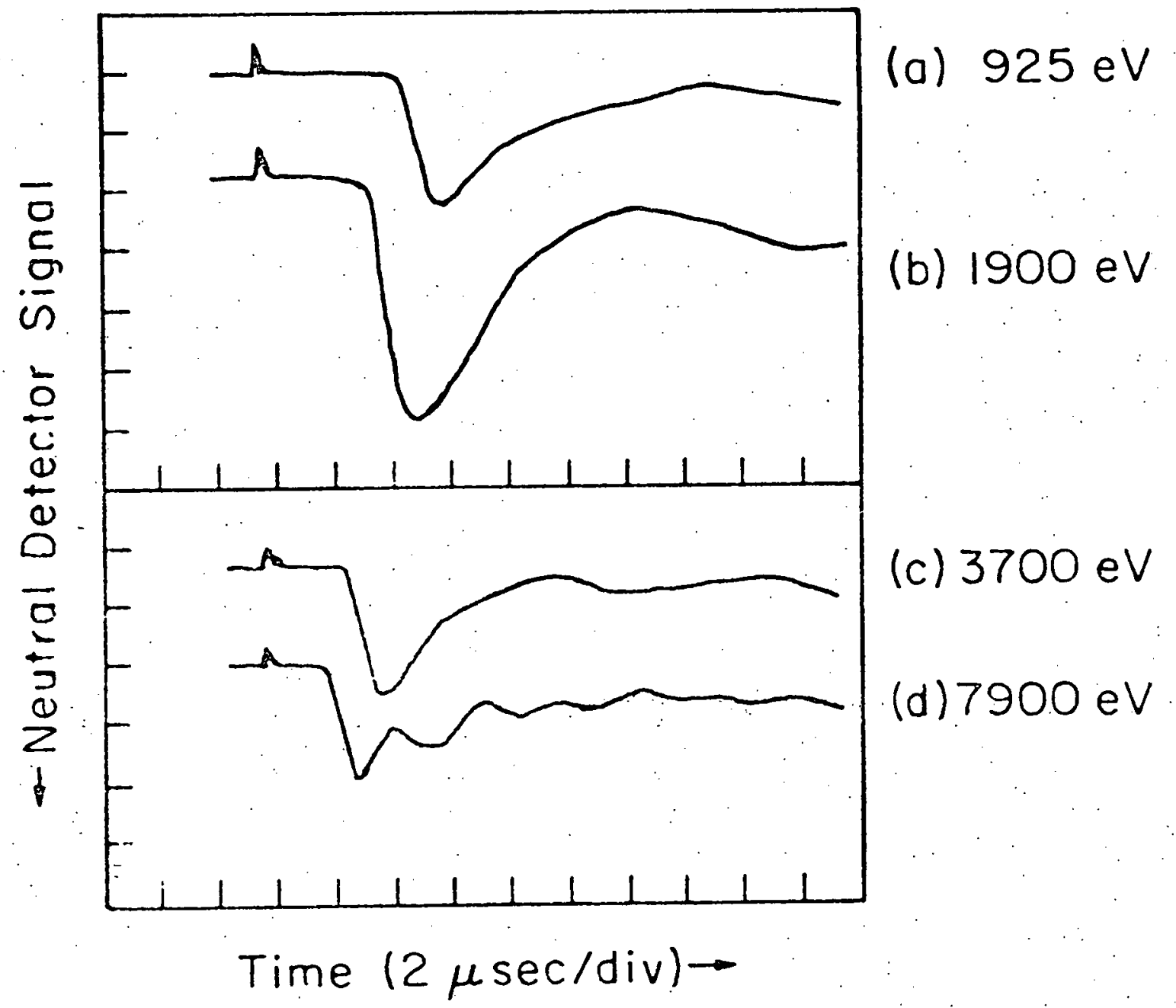

Figure 7. Neutral Signals Observed $30 \mathrm{~cm}$ From the Diode. 


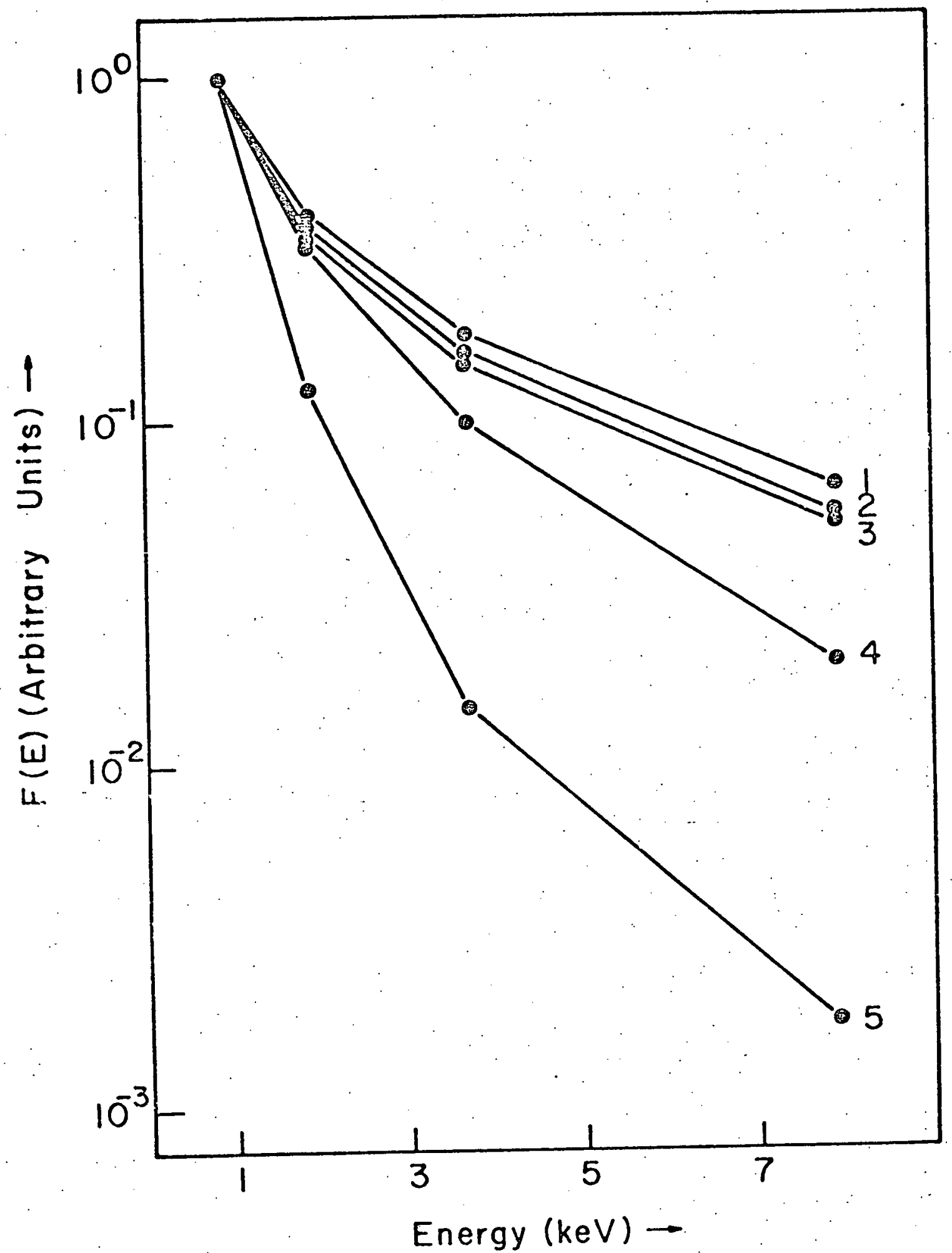

Figure 8. Ion Distribution Function Versus Energy for Various Beam Heating Levels. Curves $1-5, E_{\perp}=82,74,40,30,13 \mathrm{~J}$, respectively, $1-4 \mathrm{With} 2.5 \mathrm{~cm}$ Foilless Diode and 5 Using a $5 \mathrm{~cm}$ Foiled Diode. 

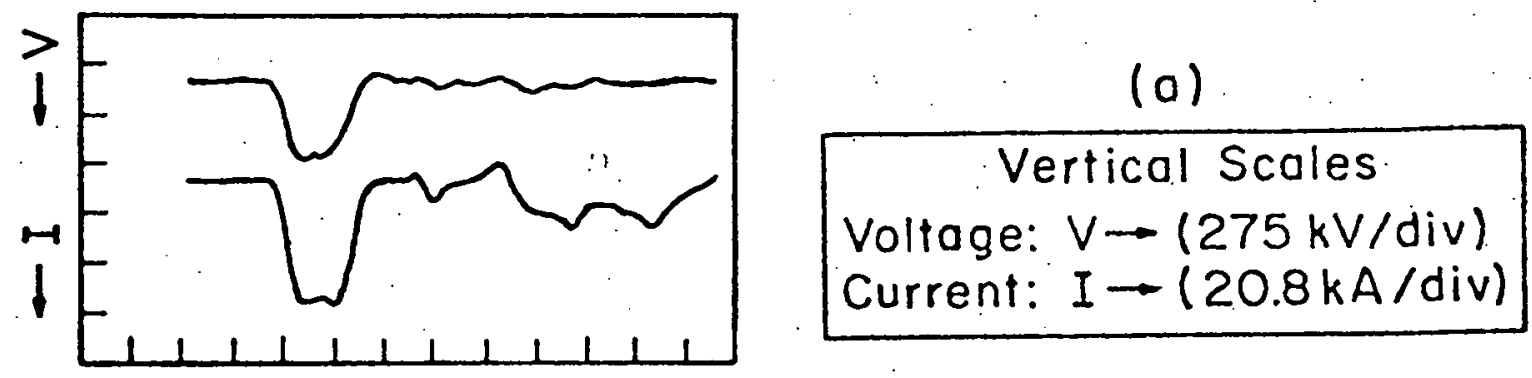

Time $\rightarrow$ (50 nsec/div)

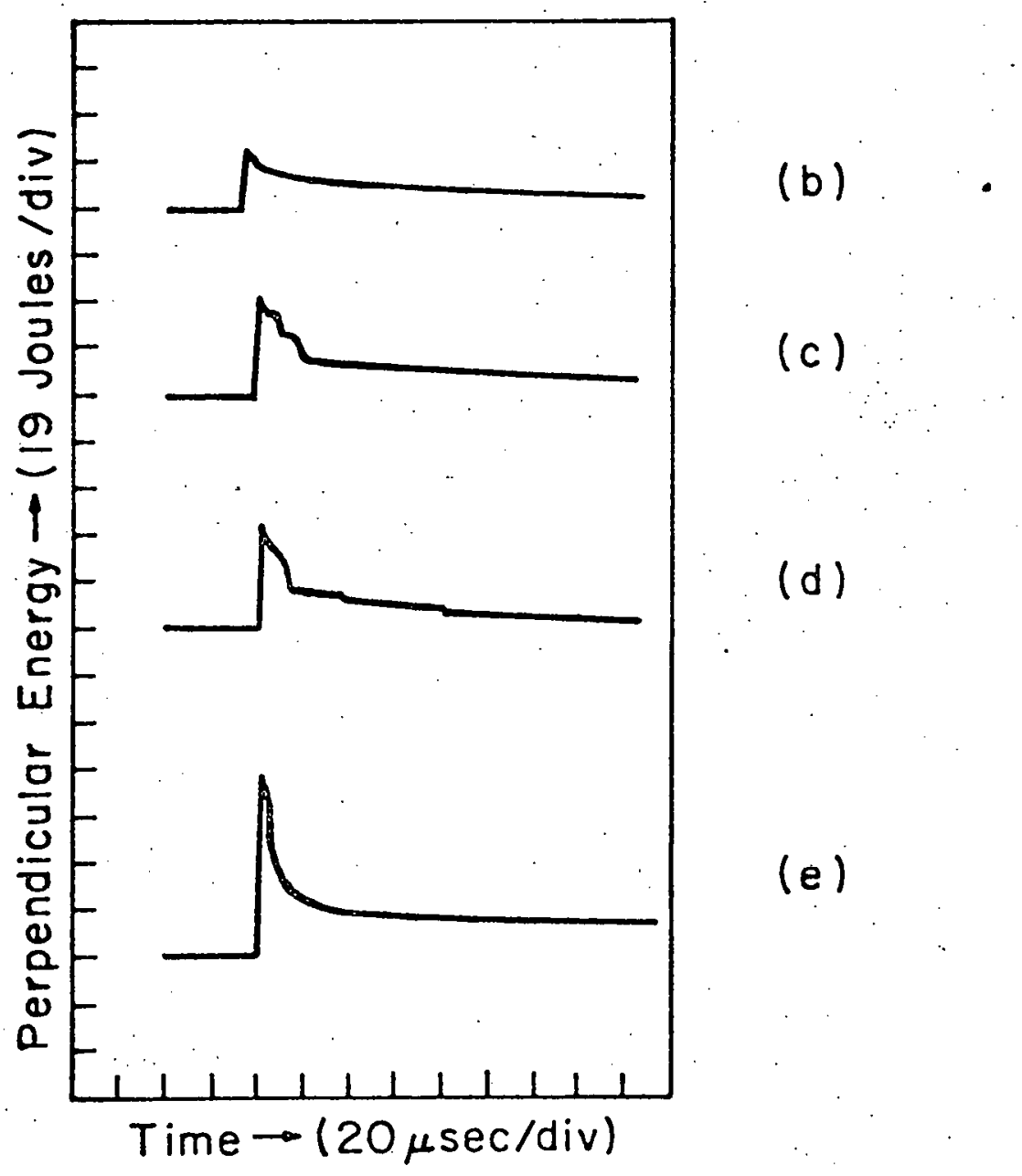

Figure 9. (a) Diode Voltage and Current Waveforms, (b) Plasma Diamagnetism, $\mathrm{E}_{1},<35 \mathrm{~J}$, (c), (d) Plasma Diamagnetism $\mathrm{E}_{\perp} \sim 35-60 \mathrm{~J}$, and (e) Plasma Diamagnetism, $\mathrm{E}_{\perp}>60 \mathrm{~J}$. 
A fourth explanation could be that the diamagnetic loop is responding to trapped relativistic electrons, although other measurements to be discussed later show no evidence of this. The second explanation seems the most plausible.

A comparison was made of beam heating and direct turbulent heating of the plasma at similar current levels. Comparative ion energy spectra obtained during a single run are shown in Figure 10. Obviously, beam heating is the more efficient process.

\section{RELATIVISTIC BEAM INTERACTION WITH A TURBULENT PLASMA}

A previous paper reported electron beam heating of a current-carrying turbulent plasma. (10) A principal result was that the heating level depended strongly on the direction of the turbulent heating current. The results could be explained by postulating the existence of a strong plasma return current. Maximum energy transfer to the plasma occurred when the beam was injected during maximum turbulence (corresponding to the peak of the turbulent heating voltage waveform) and the polarities were such that the beam return current electrons flowed in the same axial direction as the turbulent heating current electrons.

Additionally, the injection of the relativistic electron beam has been found to have a substantial effect on the effective resistance of the plasma column. Figure 11 shows the turbulent heating voltage and current oscillograms and respective diamagnetic and hard $\mathrm{x}$-ray signals for various beam injection times. The turbulent heating bank was charged to $-40 \mathrm{kV}$ in these cases. As the electron beam is injected earlier with respect to the period of peak turbulence (corresponding to the peak of the voltage waveform), the column resistance, determined from the voltage and current traces, increases and remains high for many microseconds. Also, the threshold current for turbulent heating decreases 40-50 percent, which may be a result of electron preheating by the beam.

Figure 12 is a record of two consecutive, nearly-identical shots where the beam was injected shortly after peak turbulence. In the first case, beam injection establishes a very high volumn resistance which persists for at least the duration 


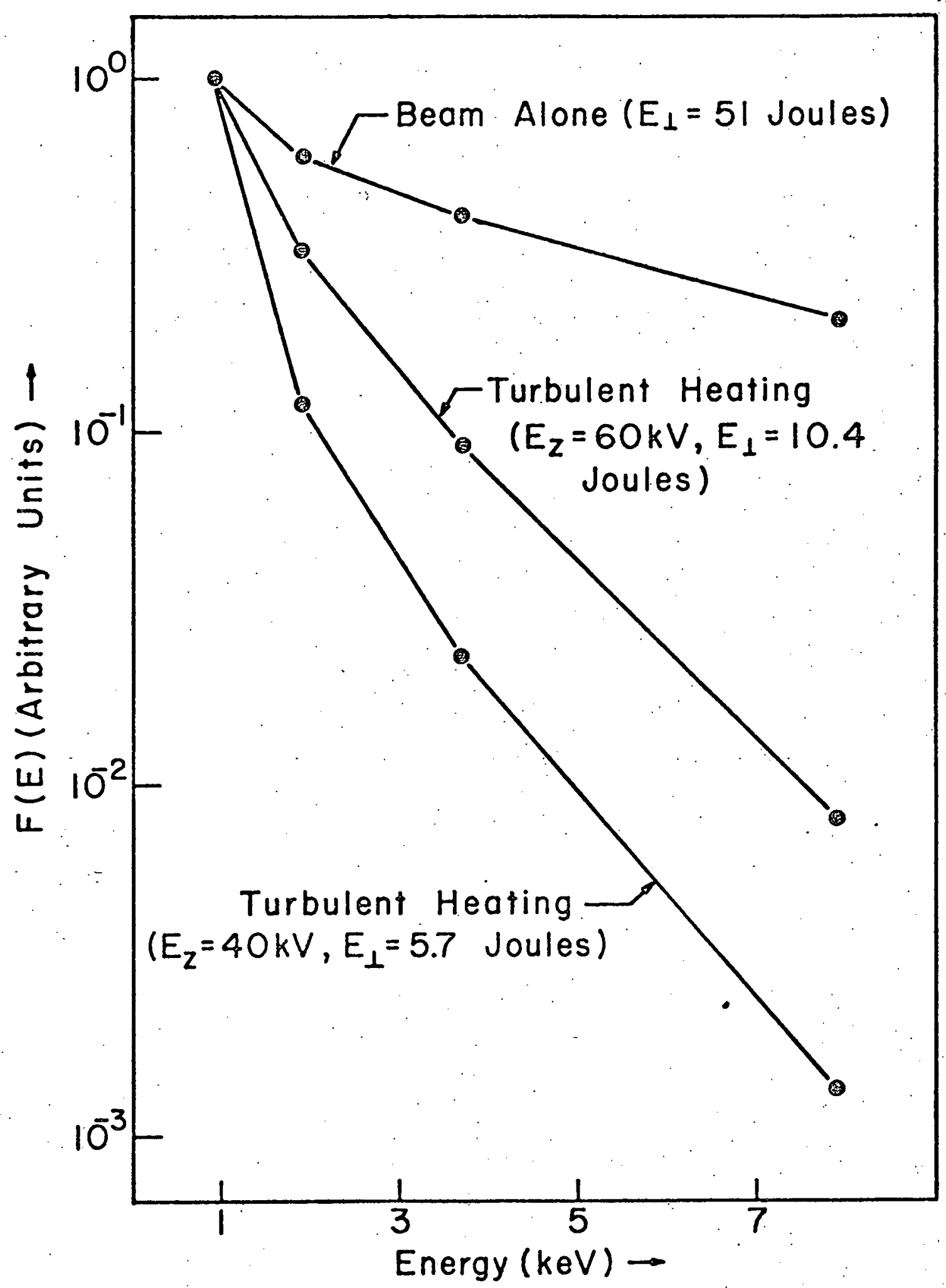

Figure 10. Comparative Neutral Energy Spectra for Beam Heating and Turbulent Heating. 

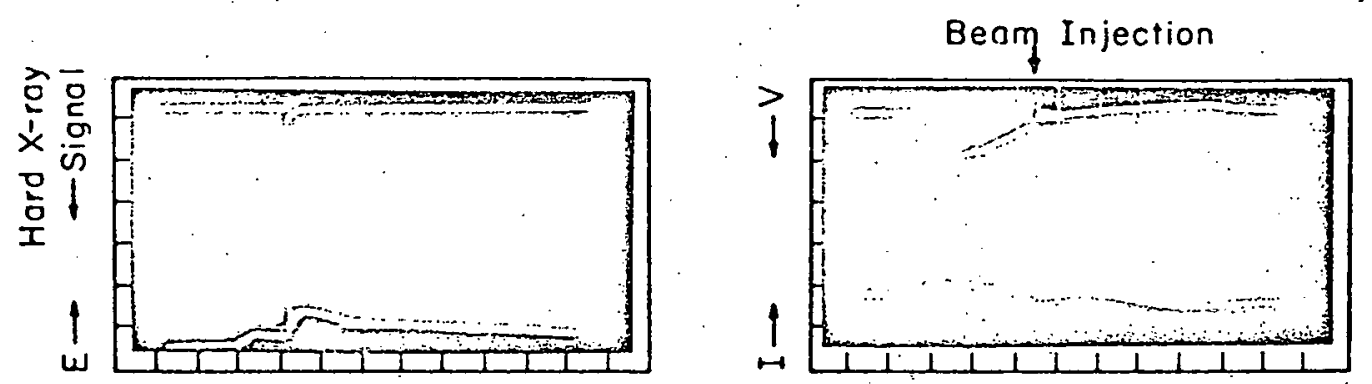

(a)
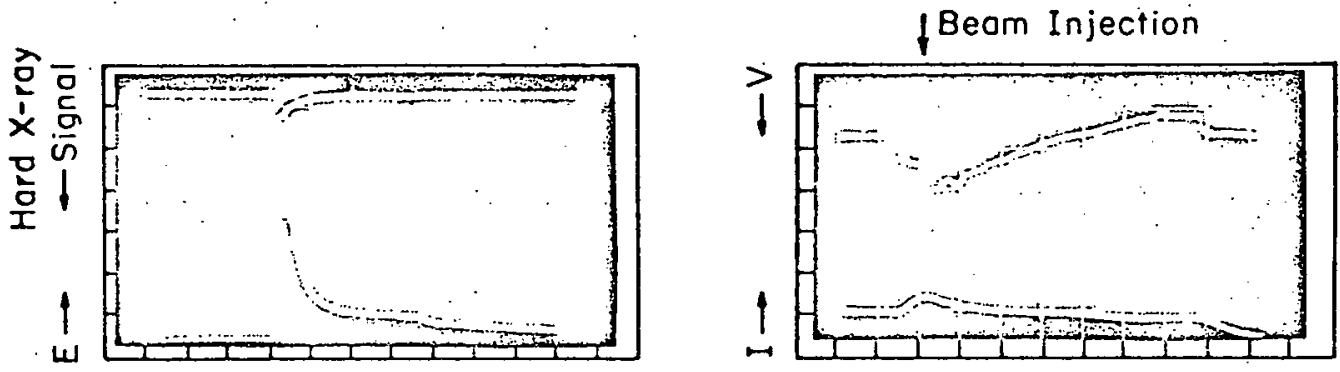

(b)
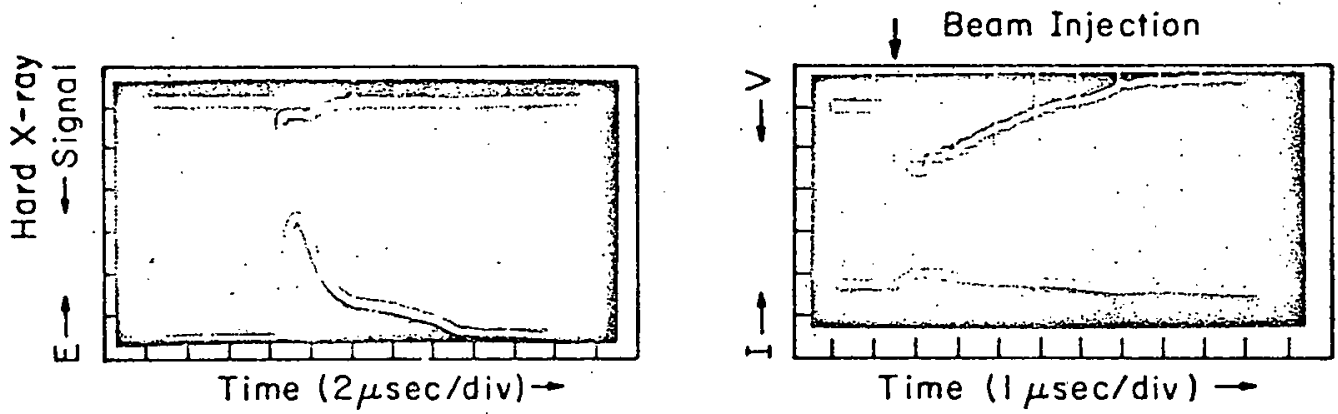

(c)

Upper: $\frac{\text { Hertical Scales }}{\text { Har ray }}$
Signal $\rightarrow(2$ V/div $)$
Lower: Diomagnetic
Signal $\rightarrow(19$ Joules/div $)$

Vertical Scoles

Voltoge: $V \rightarrow(20 \mathrm{kV} / \mathrm{div})$

Current: $I \rightarrow(14 k A / d i v)$

Figure 11. Oscilloscope Traces for the Beam - Turbulent Heating Interaction (Negative Applied Voltage). 

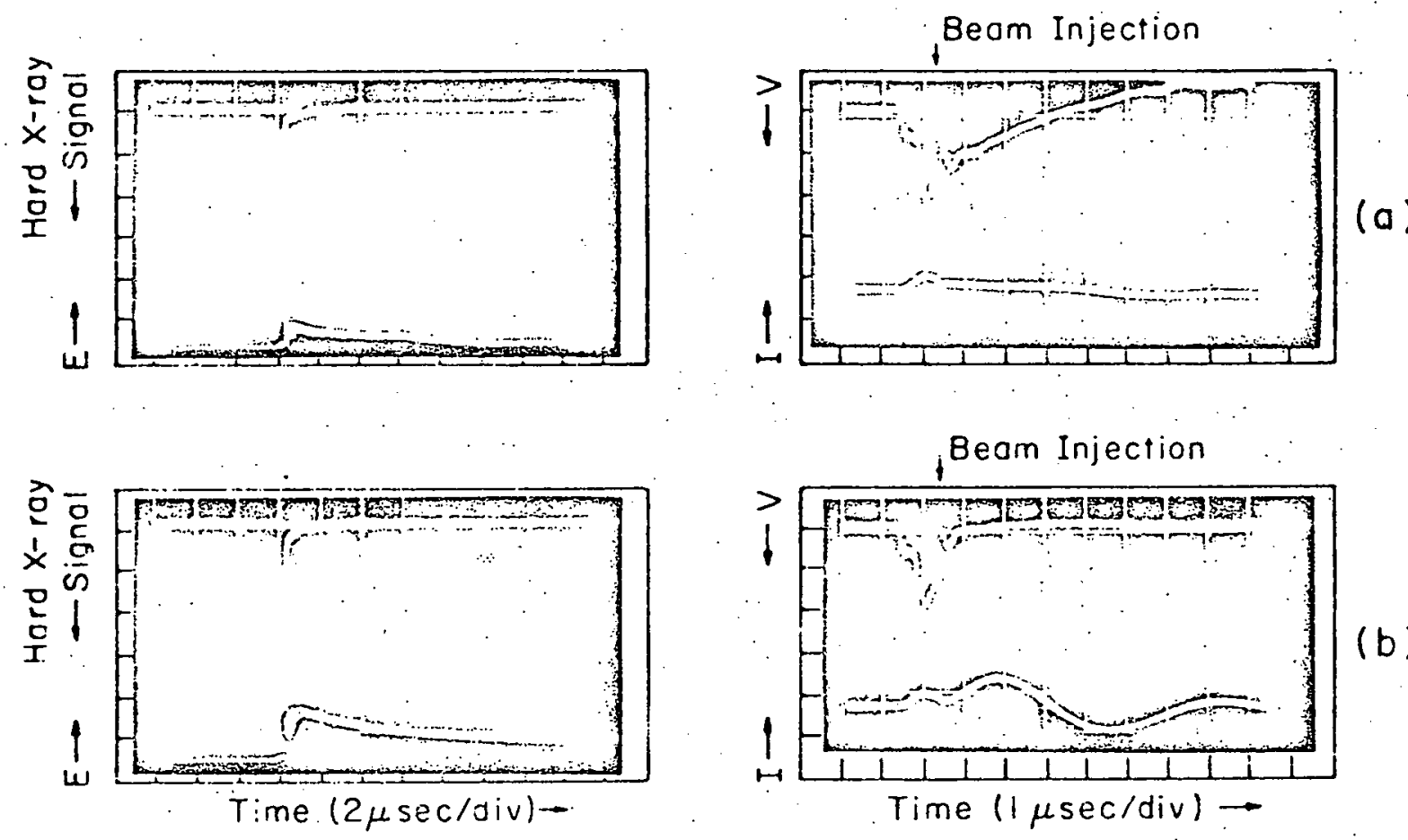

(b)

Vertical Scales
Ufper: Hord $X$-ray
Signal $\rightarrow(2 V / d i v)$
Lower: Diamagnetic
Signal $\rightarrow(19$ Joules/div $)$

$\frac{\text { Vertical Scales }}{\text { Voltage }: V \rightarrow(20 \mathrm{kV} / \text { div })}$
Current: $\mathrm{I} \rightarrow(14 \mathrm{kA} /$ div $)$

Figure 12. Effect of Beam Injection on the Highly Turbulent Plasma. 
of the oscillogram. In the next shot, a low resistance column is immediately re-established. The cause of this behavior is not yet determined. A possible explanation would be that the beam can form discontinuities in the column. If future work can show that this is not the case, but rather that the effect is an actual change in plasma resistivity, this may be important for the turbulent heating process, which is currently limited in this machine by a quenching of the currentdriven instability and the associated collapse of the anomalous resistivity after only a few hundred nanoseconds of strong heating.

On rare occasions, beam injection was found to re-initiate plasma turbulence, as shown by the second voltage spike in Figure 13. However, this was a highly nonrepeatable occurrence.

\section{EFFECT OF VARIATION OF THE MAGNETIC MIRROR FIELD}

The charging voltage of the mirror field capacitor bank could be varied to provide fields from $1.1 \mathrm{kG}$ to $2.7 \mathrm{kG}$ at the bottom of the mirror well. As shown in Figures 14 and 15, a factor of 2.5 increase in field produced only a 20 percent increase in the diode current and a factor of 2 increase in the plasma diamagnetism.

\section{TIME SCALE OF BEAM HEATING}

The diode current pulse was shortened if a conductive layer of carbon particles was allowed to build up on the lucite interface behind the diode over many shots. If this was not removed, it diverted part of the diode current by breaking down, so that the normal 60 ns trapezoidal current pulse was shortened, ultimately to a $30 \mathrm{~ns}$ triangular pulse, as increasing Marx bank voltages decreased the holdoff timc. The plasma diamagnetism versus diode current under these conditions is plotted in Figure 16. It may be concluded that the plasma heating is sensitive to the duration of the electron beam if the shape of this curve is compared with one (Figure 5) where no breakdown occurred. 

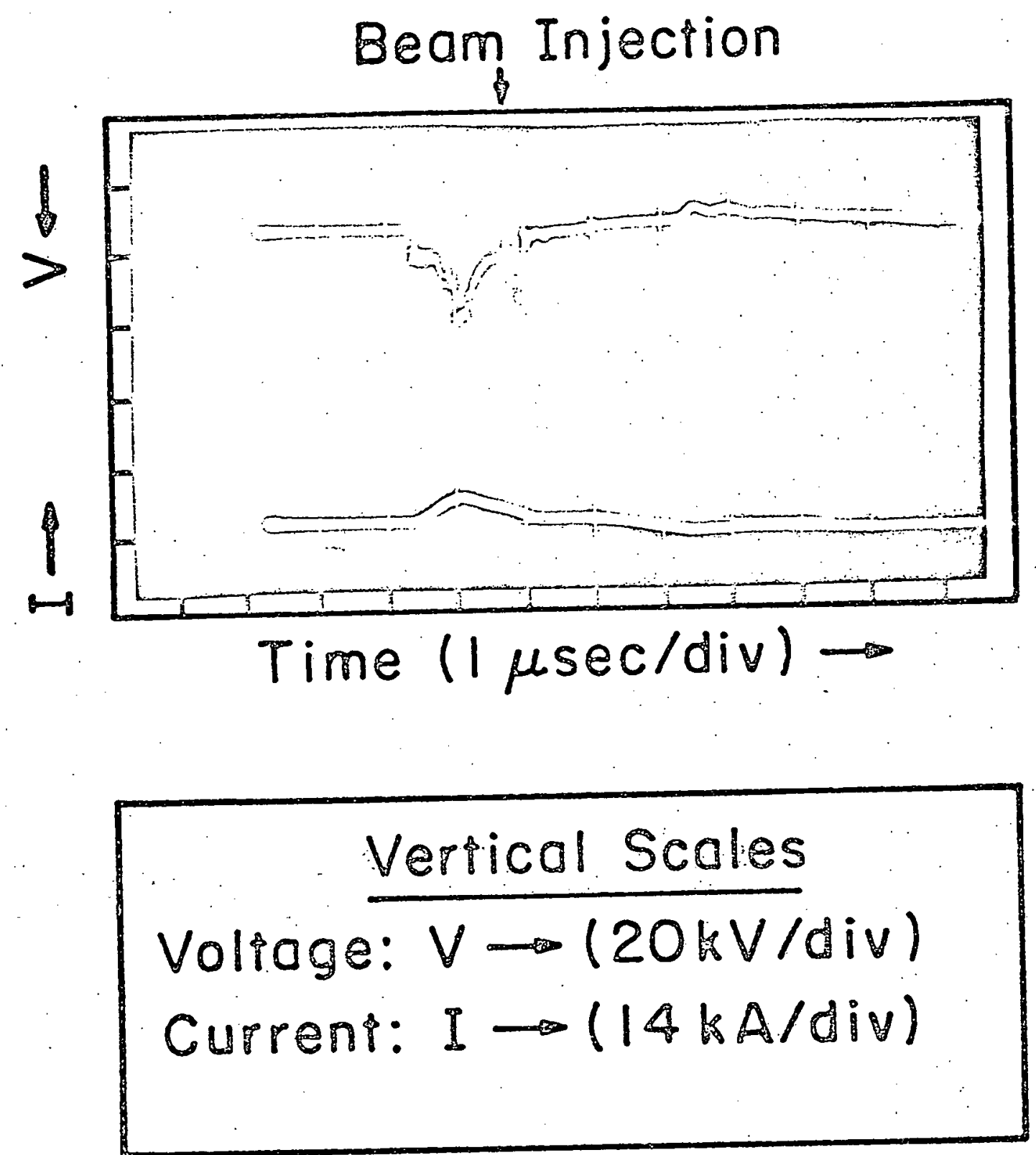

Figure 13. Beam Injection Re-initiating Plasma Turbulence. 


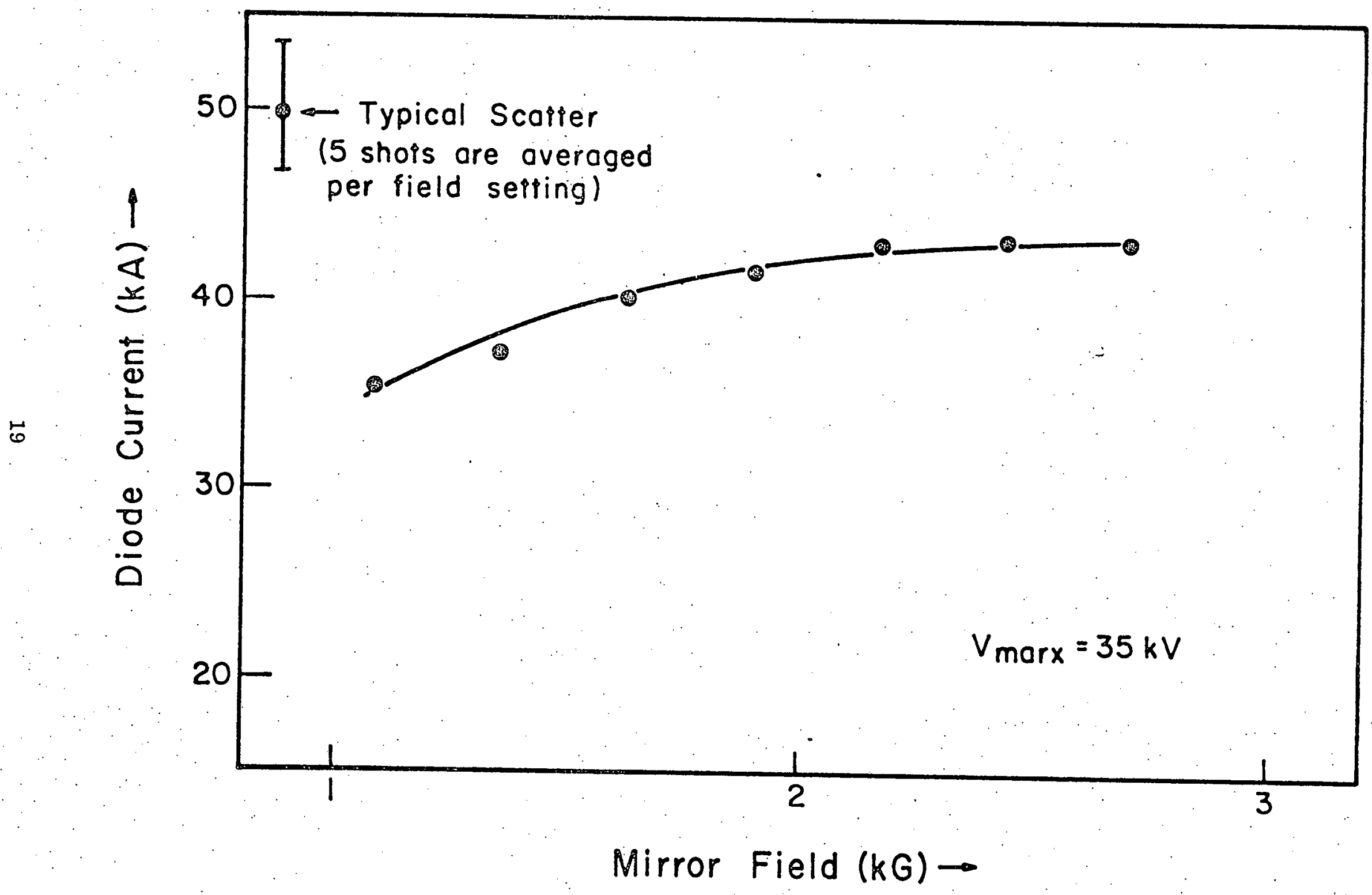

Figure 14, Diode Current Versus Magnetic Mirror Field. 


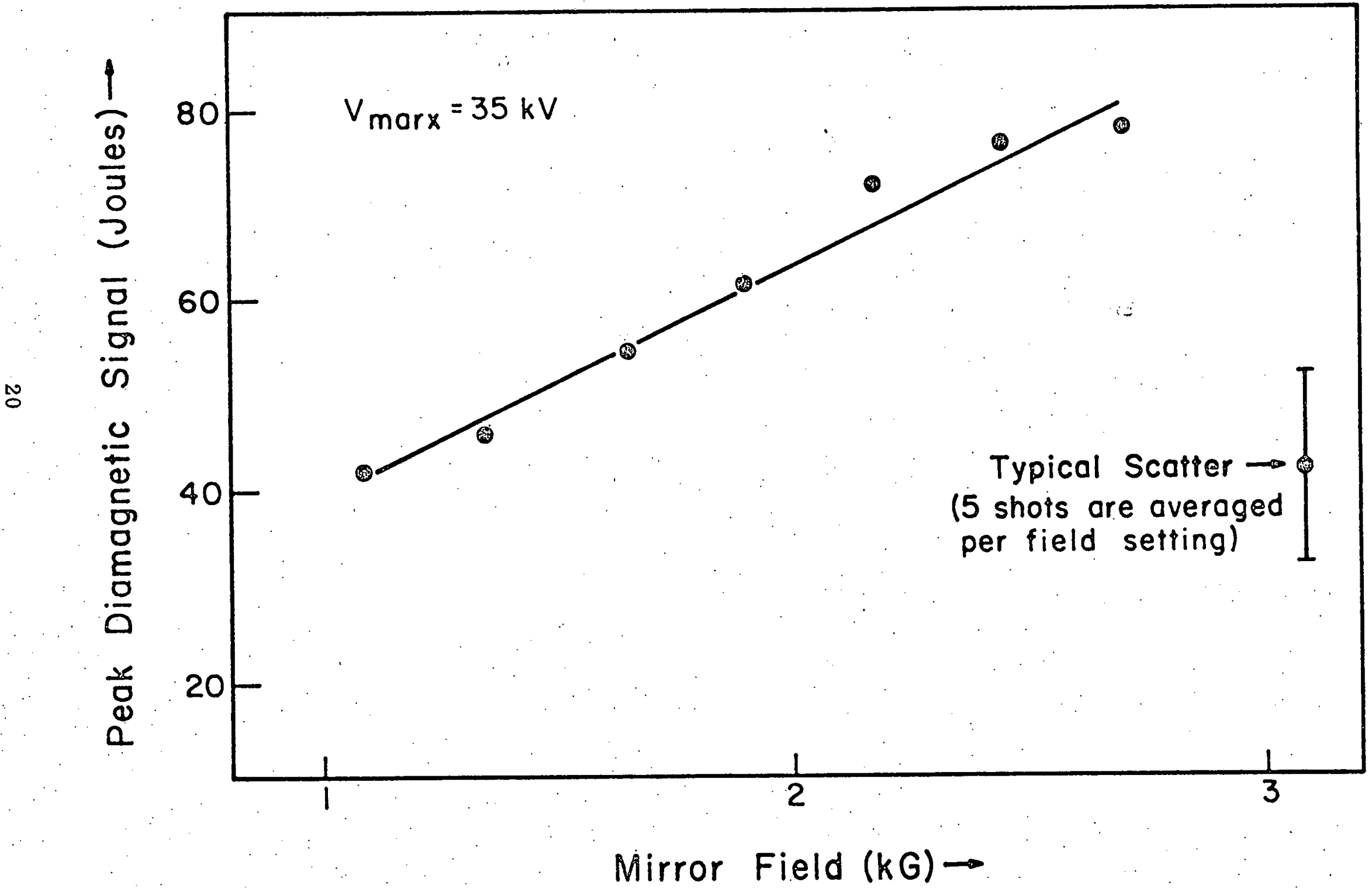

Figure 15. Peak Diamagnetic Signal Versus Magnetic Mirror Field. 


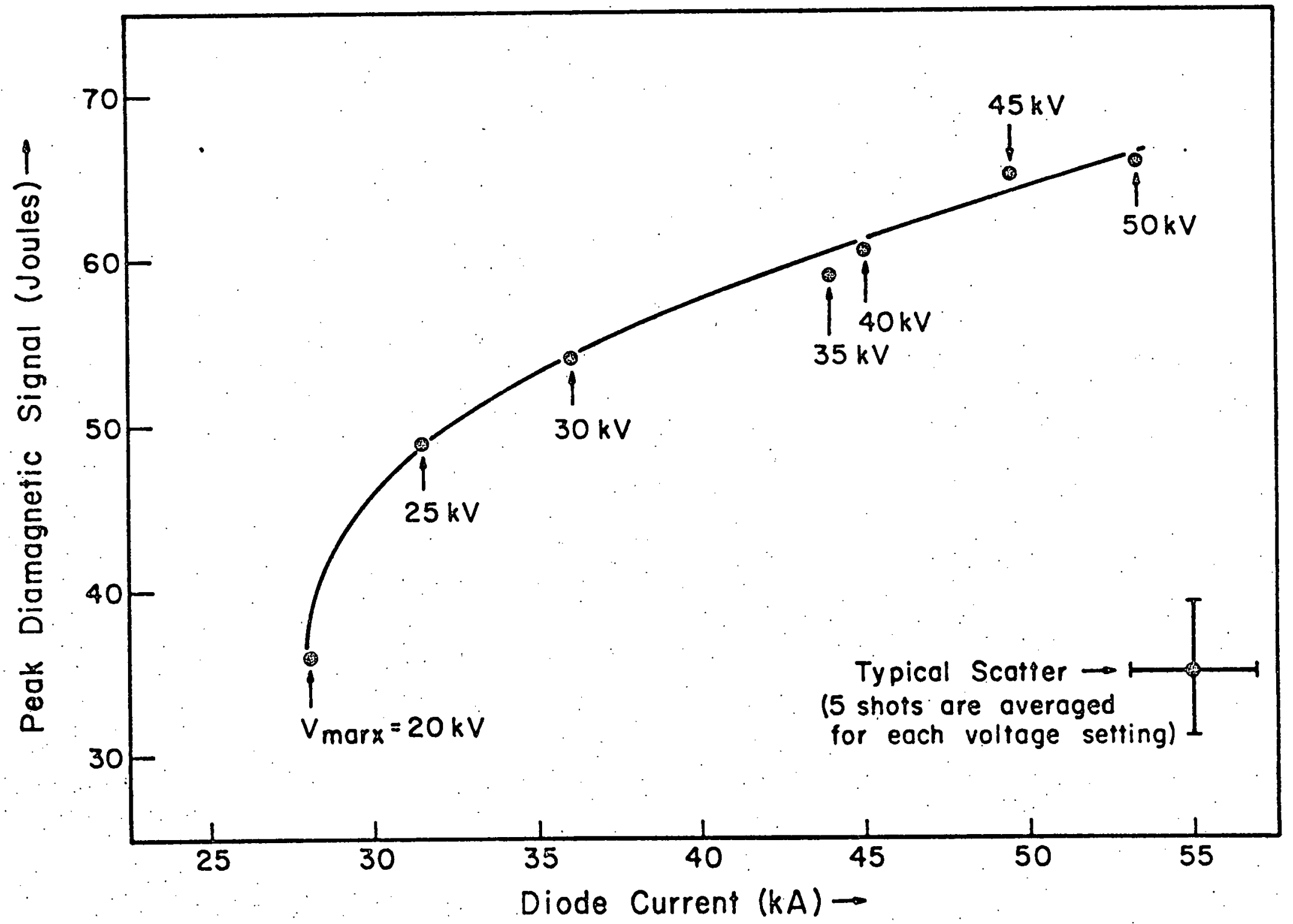

Figure 16. Peak Diamagnetic Signal Versus Diode Current (Showing the Effect of a Shortened Current Pulse). 
8. OBSERVATION OF MICROWAVE, FAR-INFRARED; AND HARD

X-RAY EMISSIONS

A microwave horn and fast $4 \mathrm{~mm}$ crystal detector, oriented to collect radiation emitted perpendicular to the mirror field at the machine midplane, polarized parallel to the magnetic field, examined broadband radiation in the vicinity of the electron plasma frequency. At the same axial position, a $2.5 \mathrm{~cm}$ diameter infrared lightguide antenna collected submillimeter radiation which was observed with a very fast helium-cooled photoconductive detector. ${ }^{(11)}$ Hard x-rays were monitored with a lead collimator aimed at the turbulent heating electrode and a scintillator-photomultiplier combination.

The peak microwave emission increased sharply with increased diode current, as shown in Figure 17. This signal increased approximately linearly with the fifth power of the magnetic mirror field, (see Figure 18). The estimated total radiated power was several kilowatts. Consideration of the parametric variations, power level, and frequency of this radiation identifies its most probable origin as nonthermal synchrotron radiation from mildly relativistic electrons. The strong dependence on magnetic field is not unprecedented, as synchrotron radiation from a hot electron plasma varying with magnetic field faster than $B^{3}$ has been reported. In addition to the normal $\mathrm{B}^{2}$ variation for synchrotron radiation, in this experiment consideration must be given to the interrelation of $\mathrm{B}$ and $\mathrm{kT}$ through the magnetic moment, and the possibility of improved plasma confinement and beam propagation as the magnetic field is increased. All of these factors contribute to the strong observed dependence.

Time-correlated oscillographs of the microwave and far-infrared emissions are shown in Figure 19. The separation of the two maxima of the microwave signal varied from shot-to-shot, as indicated in the figure. A possible explanation of the structure of the signal is considered in Section 10.

$A$ hard $\mathrm{x}$-ray burst was observed in each shot where a beam propagated into the plasma. The duration of this burst was comparable to the duration of the beam. 


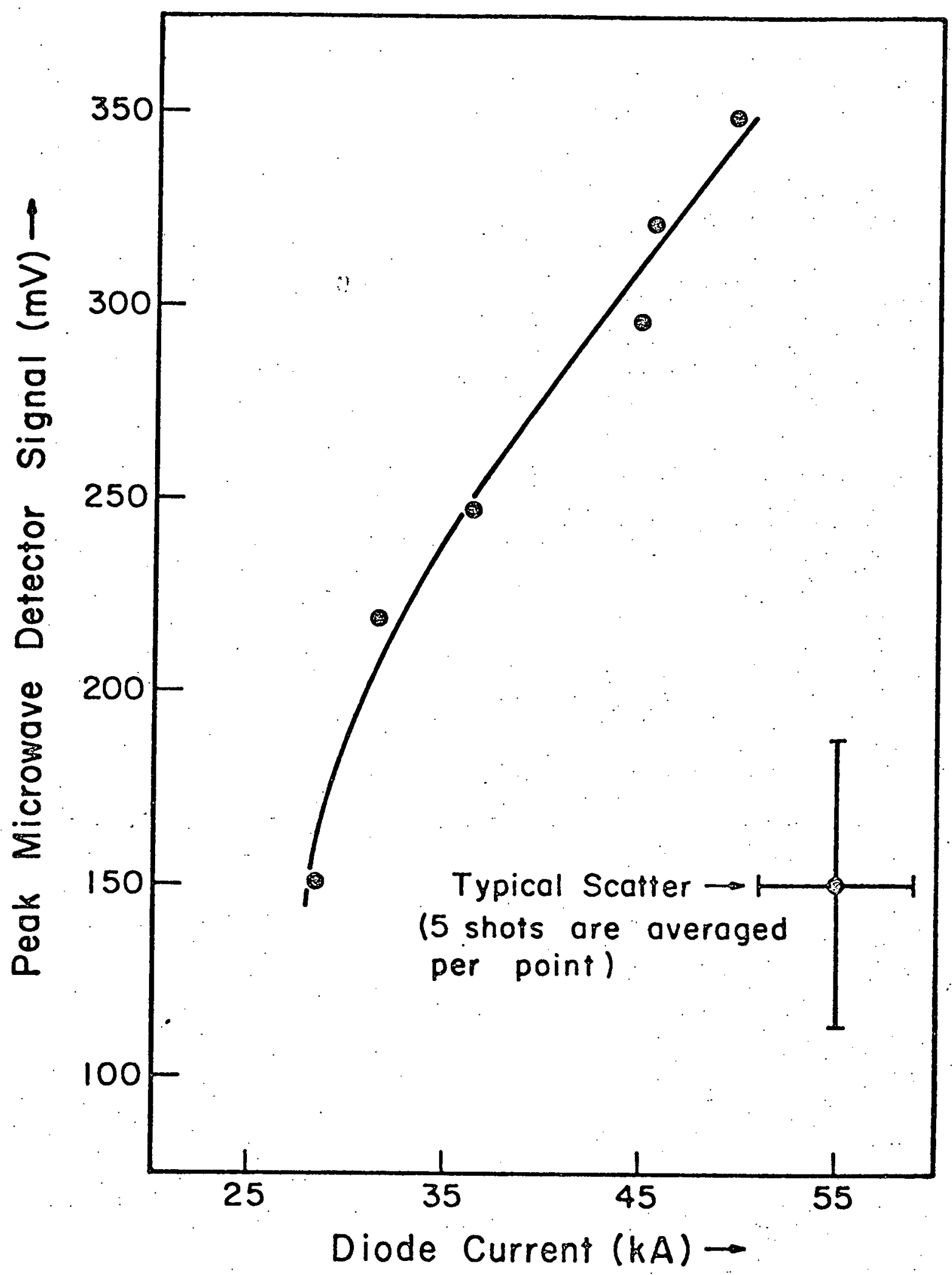

Figure 17. Peak Microwave Detector Signal Versus Diode Current. 


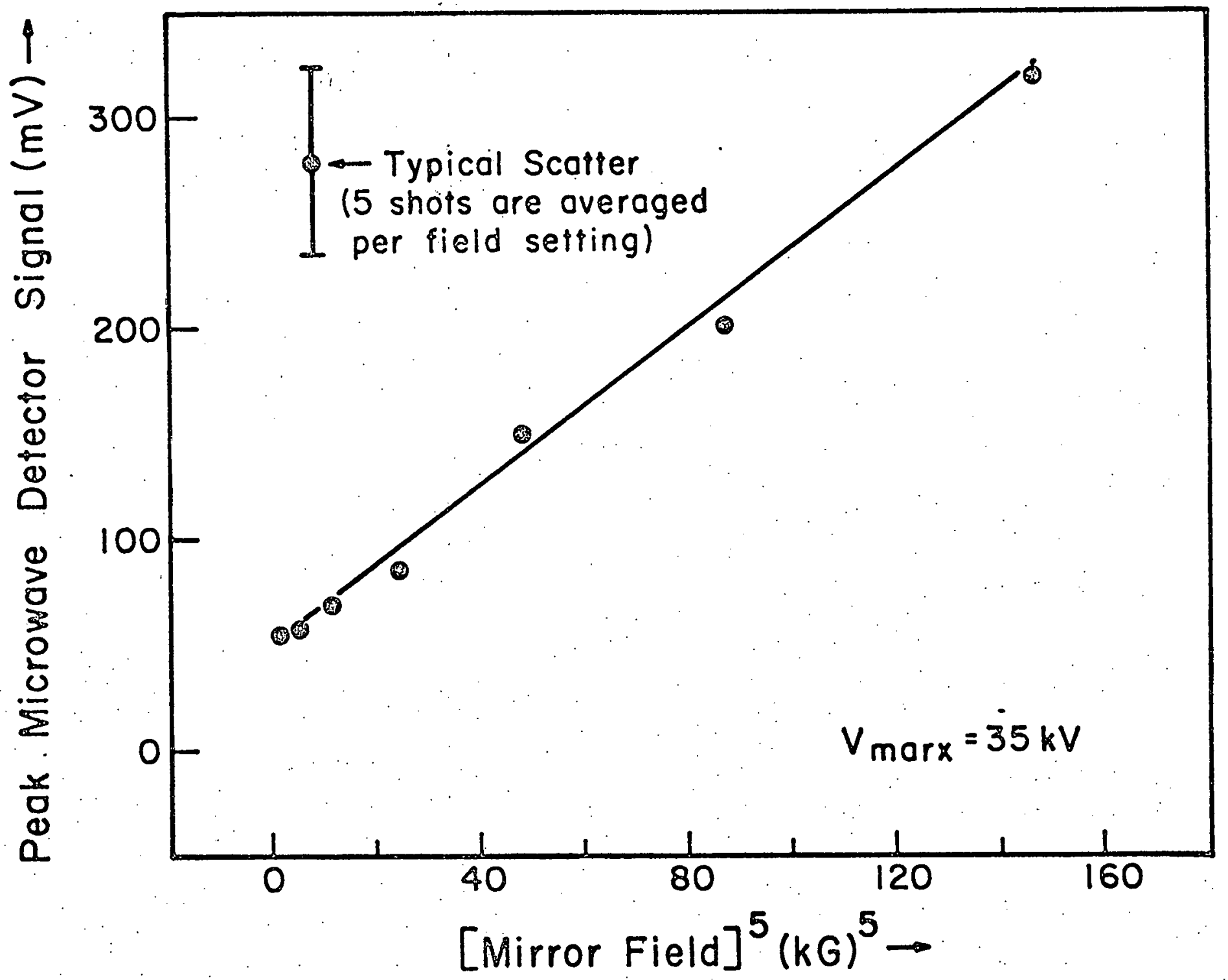

Figure 18. Peak Microwave Detector Signal Versus (Mirror Field) ${ }^{5}$. 


\section{Vertical Scales}

Upper: Far-Infrared

Defector Signal $\rightarrow(50 \mathrm{mV} /$ div $)$

Lower: Microwave

Deciect Signal $\rightarrow(500 \mathrm{mV} / \mathrm{div})$

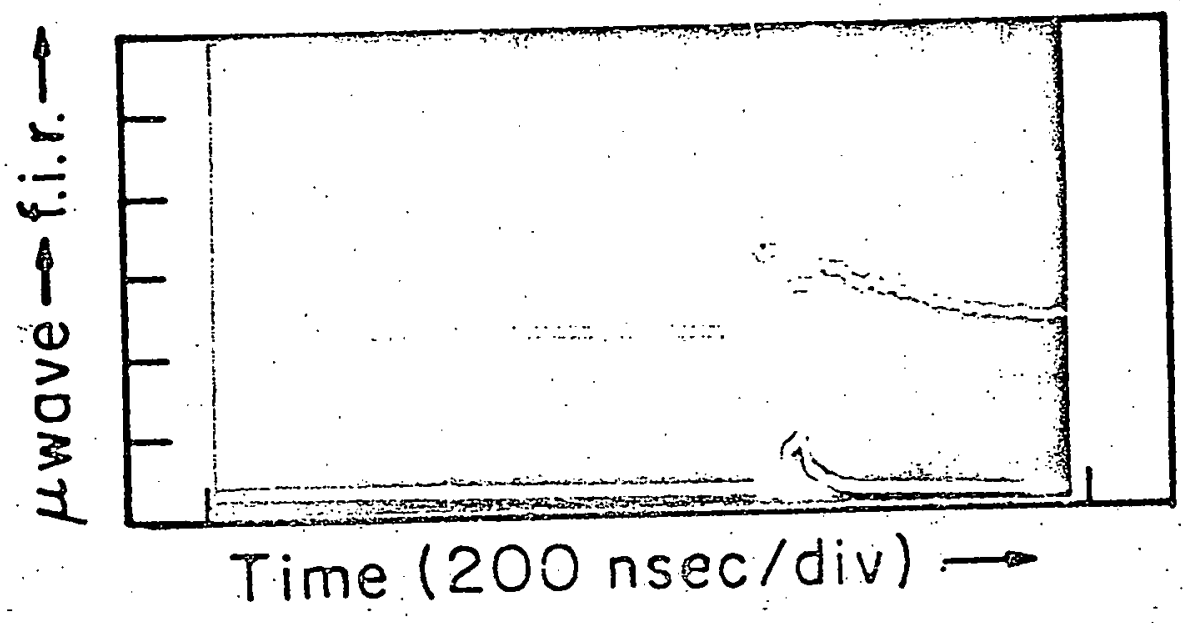

(a)

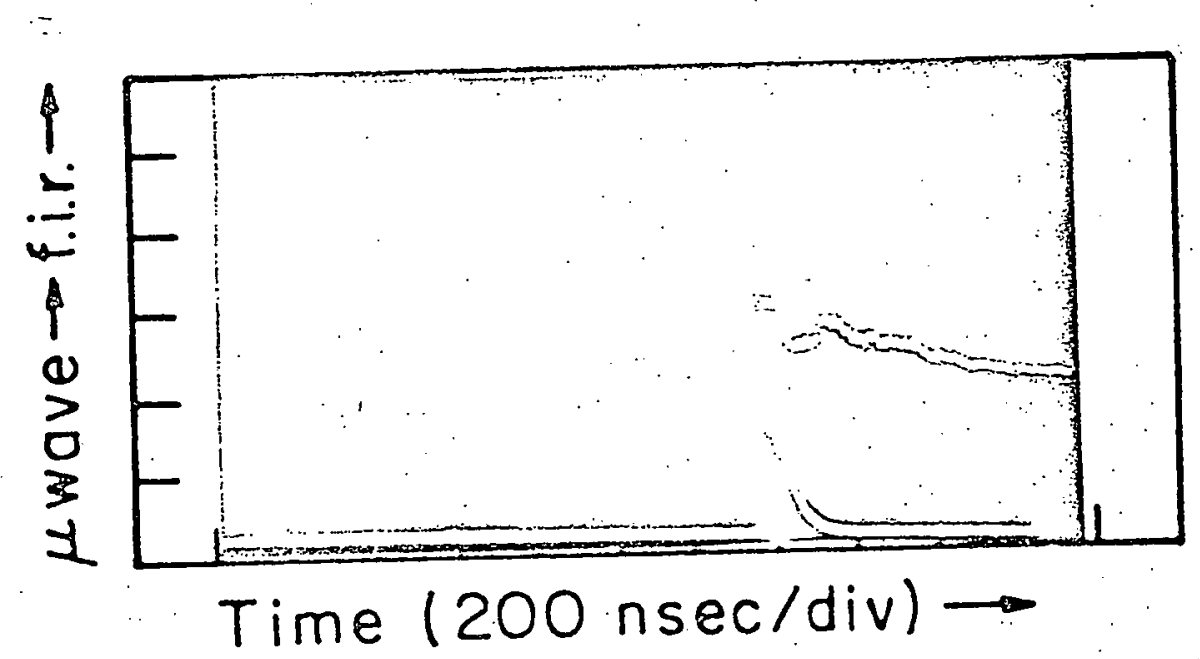

(b)

Figure 19. Time Synchronized Microwave and Far-Infrared Emission Oscillographs. 
Further bursts were observed when discontinuities in the diamagnetic signal appeared. These discontinuities seem characteristic of the heated plasina in this machine as they are also observed during direct turbulent heating. They are attributed to loss of plasma through instabilities. Several examples of this behavior are presented in Figure 20. We did not localize the source of these emissions, nor attempt to analyze the energies.

\section{LOCALIZATION OF THE HEATING PROCESS}

Several observations indicate that the bulk of the plasma heating occurs in roughly the first third of the plasma column. The neutral energy analyzer views only those particles that exit from the plasma column nearly perpendicular to the magnetic field. When the analyzer was positioned at an axial distance of $120 \mathrm{~cm}$ from the diode, neutral signals rose more slowly in time than is characteristic for this machine. However, when the analyzer was placed at a position $30 \mathrm{~cm}$ from the diode, the correct risetimes were observed. This behavior could be a result of ion heating occurring near the diode followed by diffusion of the hot plasma along the field lines.

Localized heating may contribute to the temporal structure of the microwave emission, with the initial burst corresponding to radiation from the beam electrons and the second burst resulting from hot plasma electrons, delayed because of a finite electron thermal conduction time.

Beam damage patterns in lucite tended to support the above arguments, as a strong beam was encountered only in the first third of the plasma column. These measurements were inconclusive, however, since anyobstruction to the flow of plasma into the diode severely limited beam extraction and plasma heating. As a result, Faraday cup and calorimeter measurements were not feasible, and damage patterns could be obtained only by using $0.3-0.6 \mathrm{~cm}$ lucite rods which only moderately interfered with plasma flow.

The mirror coils nearest the diode were rewired to provide the third field profile shown in Figure 3. Under these conditions, damage rods revealed that a 

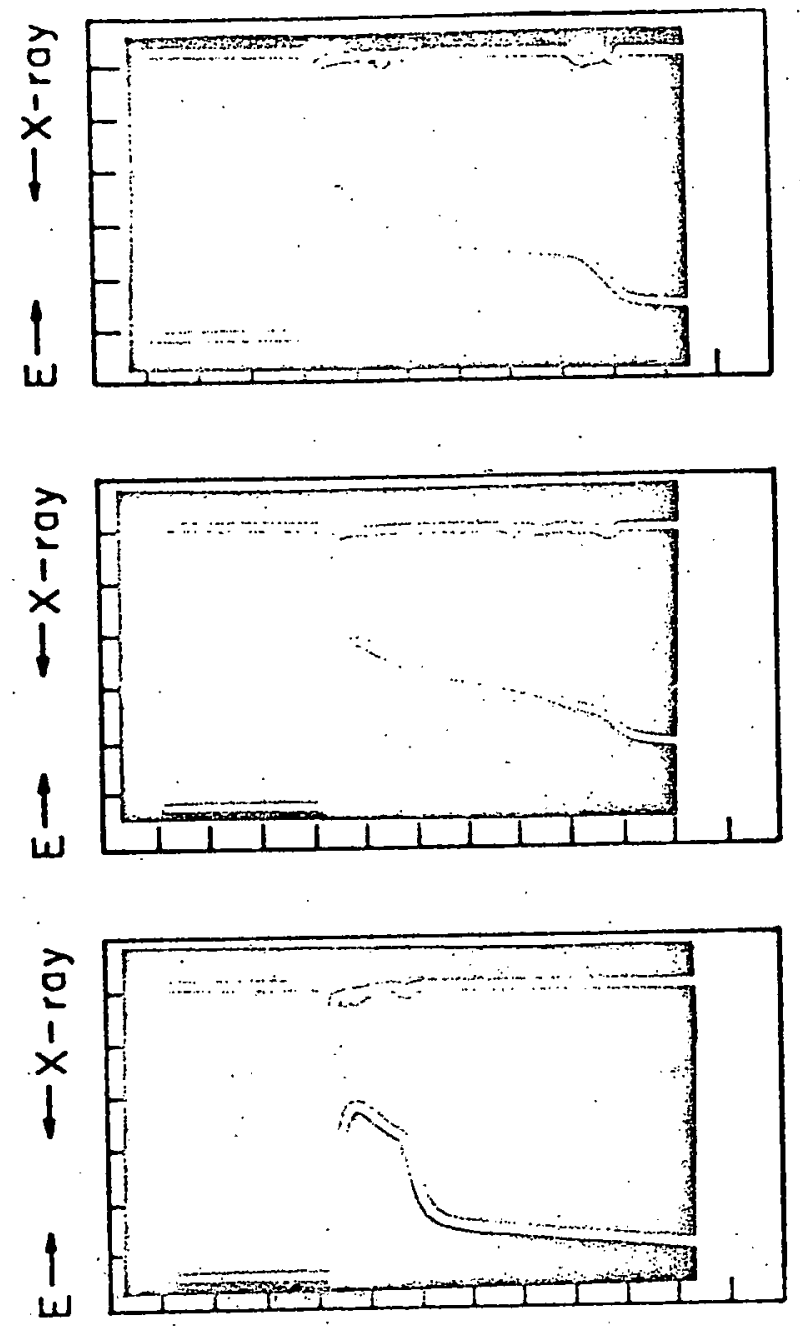

Vertical Scales
Upper: Hard X-ray
Signal $\rightarrow(2 \mathrm{~V} /$ div $)$
Lower: Diamagnetic
Signal $\rightarrow(19$ Joules/div $)$
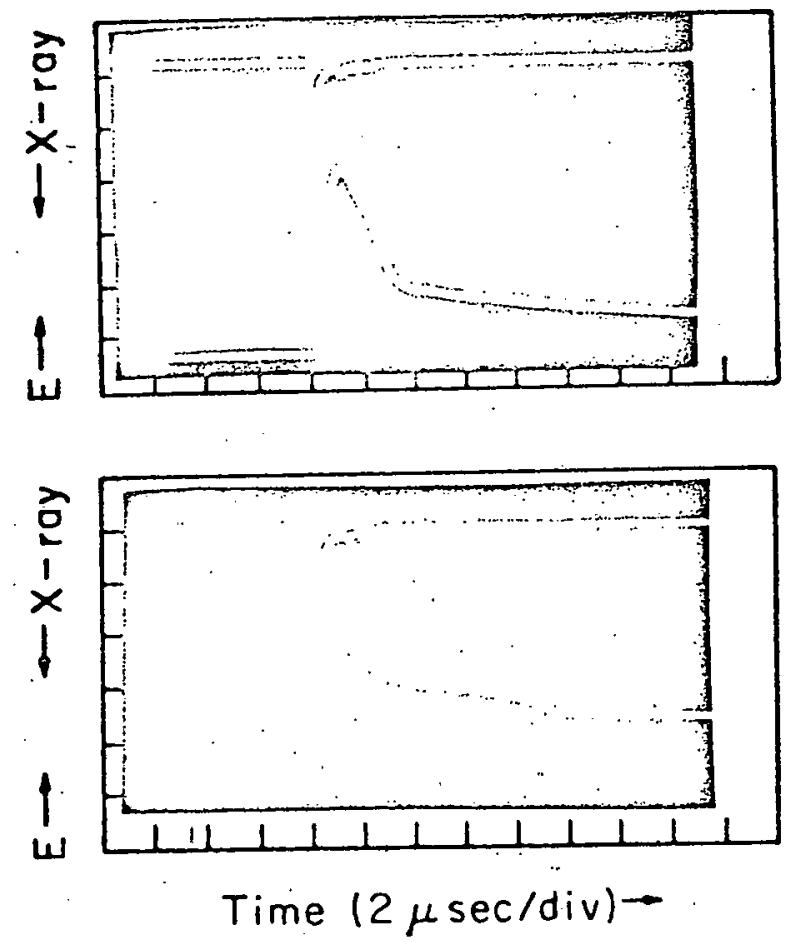

Figure 20. Hard X-Ray Emission Corresponding to Discontinuities in the Diamagnetic Signal. 
weakened beam propagated, but remained within the approximate diameter of the plasma column. This is to be contrasted to the situation above, where the fraction of the beam that reached the central portion of the mirror well was observed to . expand beyond the diameter of the plasma column. It appears, that a stronger mirror field is desirable to improve beam extraction and propagation.

\section{CONTAINMENT WITH AND WITHOUT THE MAGNETIC MIRROR}

The diamagnetic signal was observed with the normal magnetic mirror and with one mirror removed as described above. Figure 21 shows the observed decrease in the containment time without the mirror. When the $\sim 1 / 2 \mu$ sec respond time of the diamagnetic loop is considered, the decay time of the signal is appropriate to a plasma with $30 \mathrm{~J}$ of perpendicular energy ( $\sim$ average $\sim 6 \mathrm{keV}$ per electron - ion pair) escaping along the field lines at the sound speed. This indicates that the diamagnetic loop is evidently not responding to trapped relativistic electrons. It was not possible to repeat this experiment with the other mirror removed since, in that case, the gun plasma could not be successfully injected.

In support of the above argument, it should be noted that no hard $x$-ray emission was observed following the injection of the beam except when the characteristic discontinuities in the diamagnetic signal occurred. Also, as noted previously, the microwave signal decreased following the beam injection, sometimes falling to zero before the second burst. Trapped relativistic electrons would be expected to radiate at microwave frequencies continuously during this period.

\section{CONCLUSIONS AND HEATING EFFICIENCY}

It may be concluded from the experimental results that it is indeed possible to significantly heat plasma with a dense $(\nu / \gamma \geq 1)$ relativistic electron beam. Maximal energy transfer is obtained by operating the diodes at the lowest impedance level at which the beam can still be propagated. Neutral particle diagnostics indicate the presence of hot ions and show changes in the ion distribution function compatible with improved ion heating as the energy transfer to the plasma is increased. 

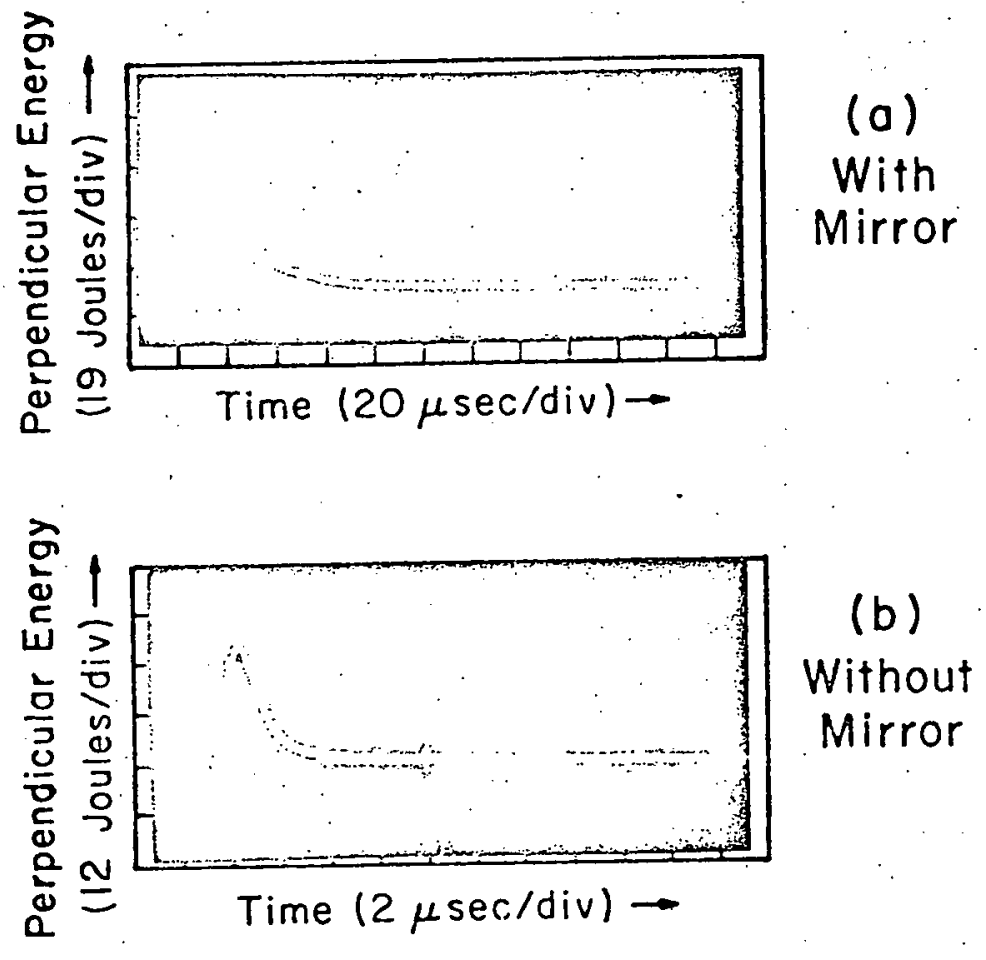

Figure 21. Plasma Diamagnetic Signal With and Without the Magnetic Mirror. 
In the regime $\nu / \gamma \leq 1$ the dominant mechanism for plasma heating is expected to be the two-stream interaction. ${ }^{(13)}$ For the conditions of this experiment $(\nu / \gamma \geq 1)$, it has been predicted ${ }^{(14)}$ that the induced plasma return current, if sufficiently strong, may excite various forms of the current driven instability. The resulting turbulent dissipation of the return current would heat the plasma at the expense of the beam energy. Some evidence of this mechanism was found in this experiment. There was a threshold current for seam heating about equal to the observed threshold for turbulent heating in this device. The character of the ion distribution function (a decrease with increasing energy much slower than a Maxwellian) was the same as that observed in turbulent heating, as were the time dependences and shapes of the diamagnetic signal and hard $x$-ray emission. Also, the beam-turbulent heating interaction was qualitatively explained by the existence of a return current.

The transfer efficiency of beam energy to plasma thermal energy cannot be quoted, since the total energy contained in the propagating beam could not be measured. However, out of a total of $\sim 700 \mathrm{~J}$ of electrical energy delivered to the diode, the diamagnetic loop indicated $70-100 \mathrm{~J}$ reliably transferred into plasma perpendicular energy, or an efficiency of $10-15$ percent. This is a considerable improvement over the 3-4 percent electrical efficiency found for direct turbulent heating in the same configuration. The respective slopes of the ion distributions differed by approximately the same ratio.

A simple calculation ${ }^{(15)}$ using the parameters of this machine predicted $75 \mathrm{~J}$ of energy transferred to the plasma on the basis of return current dissipation. This is in good agreement with the measured values.

\section{ACKNOWLEDGEMENT}

The authors wish to thank R. N. Sudan and N. Rostoker for valuable discussions, P. Brown for technical assistance, and M. Greenspan and Dr. S. Robertson for assistance with the neutral particle energy analyzer. 


\section{REFERENCES}

1. C. A. Kapetanokos and D. A. Hammer, Appl. Phys. Lett., 23, 17 (1973), and C. A. Kapetanokos et al, Bull. Amer. Phys. Soc. 17, 1032 (1972).

2. P. A. Miller and G. W. Kuswa, Phys. Rev. Lett. $\underline{30,} 958$ (1972).

3. A. T. Altyntsev et al, Pis'ma Zh. Eksp. Teor. Fiz. 13, 197 (1971). JETP Lett. 13, 139 (1971), and Proceedings of the Fourth International Conference on Plasma Physics and Controlled Nuclear Fusion Research, Madison, Wisconsin, 1971 (International Atomic Energy Agency, Vienna, 1972), Vol. 2, p. 309.

4. G. C. Goldenbaum et al, Bull. Amer. Phys. Soc. 17, 1031 (1972).

5. C. Stallings et al, Bull. Amer. Phys. Soc. 17, 1031 (1972).

6. C. B. Wharton et al, Proc. 4th Intl. IAEA Conf. on Plasma Physics and Controlled Thermonuclear Fusion Research, Paper CN28/E2 (Madison, Wisc. 1971). Vol. II, p 25.

7. P. Korn, F. Sandel, and C. B. Wharton, Phys. Rev. Lett. 1 1, 579 (1973).

8. S. Robertson, P. Korn and C. B. Wharton, IEEE Trans. Plasma Science, PS-2. 17 (1973).

9. S. Robertson et al, to be published in Rev. Sci. Instr.

10. P. Korn, F. Sandel, and C. B. Wharton, to be published in J. Appl. Phys. Nov. 1973.

11. F. Sandel, Ph.D. Thesis, Cornell University, Ithaca, N.Y. (1973).

12. A. J. Lichtenberg et al, Phys. Fluids $\underline{7}, 1549$ (1964).

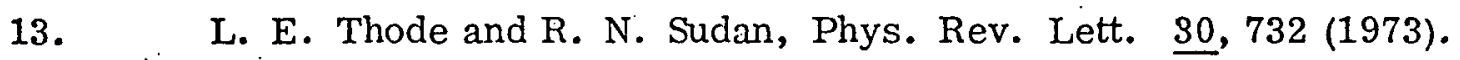

14. R. V. Lovelace and R. N. Sudan, Phys. Rev. Lett. 27, 1256 (1971).

15. Communication with N. Rostoker. 